\title{
Smooth 2-Group Extensions and Symmetries of Bundle Gerbes
}

\author{
Severin Bunk ${ }^{1}$, Lukas Müller ${ }^{2}$ D, Richard J. Szabo ${ }^{3,4,5}$ \\ ${ }^{1}$ Fachbereich Mathematik, Bereich Algebra und Zahlentheorie, Universität Hamburg, Bundesstraße 55, \\ 20146 Hamburg, Germany. E-mail: severin.bunk@uni-hamburg.de \\ 2 Max-Planck-Institut für Mathematik, Vivatsgasse 7, 53111 Bonn, Germany. \\ E-mail: lmueller4@mpim-bonn.mpg.de \\ 3 Department of Mathematics, Heriot-Watt University, Colin Maclaurin Building, Riccarton, Edinburgh EH14 \\ 4AS, UK. \\ 4 Maxwell Institute for Mathematical Sciences, Edinburgh, UK. \\ 5 Higgs Centre for Theoretical Physics, Edinburgh, UK. E-mail: r.j.szabo@hw.ac.uk
}

Received: 13 May 2020 / Accepted: 16 April 2021

Published online: 25 May 2021 - (C) The Author(s) 2021

\begin{abstract}
We study bundle gerbes on manifolds $M$ that carry an action of a connected Lie group $G$. We show that these data give rise to a smooth 2-group extension of $G$ by the smooth 2-group of hermitean line bundles on $M$. This 2-group extension classifies equivariant structures on the bundle gerbe, and its non-triviality poses an obstruction to the existence of equivariant structures. We present a new global approach to the parallel transport of a bundle gerbe with connection, and use it to give an alternative construction of this smooth 2-group extension in terms of a homotopy-coherent version of the associated bundle construction. We apply our results to give new descriptions of nonassociative magnetic translations in quantum mechanics and the Faddeev-Mickelsson-Shatashvili anomaly in quantum field theory. We also propose a definition of smooth string 2-group models within our geometric framework. Starting from a basic gerbe on a compact simply-connected Lie group $G$, we prove that the smooth 2-group extensions of $G$ arising from our construction provide new models for the string group of $G$.
\end{abstract}

\section{Contents}

1. Introduction . . . . . . . . . . . . . . . . . 1830

2. Preliminaries on Diffeological Spaces and Gerbes . . . . . . . . . . . . 1833

2.1 Diffeological spaces . . . . . . . . . . . . . . . . . . . . 1833

2.2 Bundle gerbes and transgression . . . . . . . . . . . . . . . . 1835

3. Group Extensions from Principal Bundles . . . . . . . . . . . . . . . . 1838

3.1 Global description . . . . . . . . . . . . . . . . . . . . . . . 1839

3.2 Equivariant bundles . . . . . . . . . . . . . . . . . . . . . . 1842

3.3 Description via parallel transport . . . . . . . . . . . . . . . . . . . . 1843

4. A Global Approach to Parallel Transport for Bundle Gerbes . . . . . . . . . 1846

4.1 A path space approach to parallel transport on line bundles . . . . . . 1846

4.2 Global definition of parallel transport on bundle gerbes . . . . . . . . 1849

4.3 Construction of the parallel transport . . . . . . . . . . . . . . . 1852 
4.4 The transgression line bundle as a holonomy . . . . . . . . . . . . . . 1860

5. 2-Group Extensions from Bundle Gerbes . . . . . . . . . . . . . . . . 1861

5.1 Smooth groupoids and symmetries of gerbes . . . . . . . . . . . . 1862

5.2 Smooth 2-groups . . . . . . . . . . . . . . . . . . . . 1865

5.3 Smooth principal 2-bundles . . . . . . . . . . . . . . . . 1866

5.4 Global description of the 2 -group extension . . . . . . . . . . . . . 1870

5.5 Descent description of the 2-group extension . . . . . . . . . . . . . . 1874

5.6 Equivariant bundle gerbes . . . . . . . . . . . . . . . . . . . . . 1880

6. Application I: Nonassociative Magnetic Translations . . . . . . . . . . . . 1885

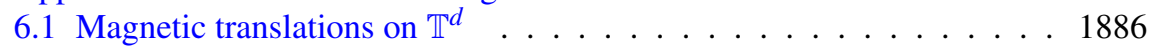

6.2 Nonassociative magnetic translations from parallel transport . . . . . 1888

6.3 Nonassociative magnetic translations on $\mathbb{T}^{d} \ldots$. . . . . . . . . . . . 1891

7. Application II: Anomalies in Quantum Field Theory . . . . . . . . . . . . 1894

7.1 Even dimensions: chiral anomalies . . . . . . . . . . . . . . . . . . . 1894

7.2 Odd dimensions: the Faddeev-Mickelsson-Shatashvili anomaly . . . 1895

8. Application III: The String Group . . . . . . . . . . . . . . . . . . . . . . 1898

8.1 A smooth string group model . . . . . . . . . . . . . . . . . . . . . 1898

8.2 Smooth string 2-group models . . . . . . . . . . . . . . . . . . 1899

A. Properties of Smooth Principal 2-Bundles . . . . . . . . . . . . . . . . 1905

A.1 Surjectivity on objects and homotopy pullbacks . . . . . . . . . . . . 1905

A.2 Relation to principal $\infty$-bundles . . . . . . . . . . . . . . . . 1907

\section{Introduction}

This paper is motivated by the following problem from physics: In [BMS19] we showed how a bundle gerbe with connection on $\mathbb{R}^{d}$ gives rise to a 3-cocycle on the translation group $\mathbb{R}_{\mathrm{t}}^{d}$ of $\mathbb{R}^{d}$. Even though this 3-cocycle is trivial in group cohomology, it is very interesting from a physical as well as from a mathematical perspective: it gives a geometric explanation to the presence of nonassociativity in quantum mechanics with magnetic monopole backgrounds, and it implements the action of the parallel transport of a bundle gerbe on its 2-Hilbert space of sections. This appearence of nonassociativity in quantum mechanics goes back to [Jac85, GZ86], but as of yet the more natural extension to realistic scenarios involving periodically confined motion on configuration spaces such as tori $\mathbb{T}^{d}$ has not been worked out. The discussion of [Jac85] was a response to the observed violation of the Jacobi identity for the algebra of field operators in quantum gauge theories with chiral fermions [Jo85], which is a manifestation of the chiral anomaly. Interest in these models has been recently revived through their conjectural relevance to nongeometric flux compactifications of string theory, which is based on backgrounds that are tori or more generally torus bundles [Lüs10, MSS12, BL14, MSS14]. However, the original finding [BP11] of nonassociativity in Wess-Zumino-Witten models based on other compact Lie groups has so far received considerably less attention, and in particular has not been understood from a geometric perspective.

In the present paper we work out the geometric framework and origin behind these results in complete generality. Subsequently, we present several applications of our results in both physics and mathematics, along the lines discussed above. We consider an action $\Phi: G \times M \longrightarrow M$ of a connected Lie group $G$ on a manifold $M$, where $M$ is endowed with a bundle gerbe $\mathcal{G}$. One can now ask whether $\mathcal{G}$ admits a $G$-equivariant structure. At the very least, such a structure should consist of a choice of 1-isomorphism 
$\mathcal{G} \longrightarrow \Phi_{g}^{*} \mathcal{G}$ for every $g \in G$. Instead of considering possible choices for such 1 isomorphisms individually, we assign to $g$ the groupoid of all such 1-isomorphisms. This yields an object which can be understood as a bundle $\operatorname{Sym}_{G}(\mathcal{G}) \longrightarrow G$ of groupoids over $G$. Considering $g=e$, the identity element of $G$, we see that its typical fibre is the groupoid $\operatorname{HLBdl}(M)$ of hermitean line bundles on $M$.

The definition of $\operatorname{Sym}_{G}(\mathcal{G})$ so far does not capture the smooth structure of the gerbe $\mathcal{G}$. We thus enhance the construction to take into account smooth families of elements of $G$. Then one can make sense of $\operatorname{Sym}_{G}(\mathcal{G})$ as a category fibred in groupoids over a base category Cart that encodes smooth families of geometric objects. Categories fibred in groupoids over Cart assemble into a 2-category $\mathcal{H}$, and there exists a fully faithful inclusion of the category of smooth manifolds into $\mathcal{H}$. Motivated by [SP11] we define a smooth 2-group to be a group object in $\mathcal{H}$. One of the central examples for us is the smooth 2-group $\mathrm{HLBdl}^{M}$ of hermitean line bundles on $M$. We introduce a notion of smooth principal 2-bundle in $\mathcal{H}$ that lies between the definitions of higher principal bundles used in [SP11] and [NSS15] (see in particular Appendix A.2). We show that our principal 2-bundles are well behaved from a homotopical as well as from a geometric point of view (more precisely, they form effective epimorphisms while also admitting local sections). With the notion of smooth 2-group and principal 2-bundles, we can make precise what it means to be a (central) extension of smooth 2-groups in analogy to extensions of Lie groups. Then, our first main results can be summarised as

Theorem 1.1. Let $G$ be a connected Lie group acting on a manifold $M$, and let $\mathcal{G}$ be a bundle gerbe on M. Then:

(1) There is a (non-central) extension of smooth 2-groups

$$
1 \longrightarrow \operatorname{HLBdl}^{M} \longrightarrow \operatorname{Sym}_{G}(\mathcal{G}) \longrightarrow \underline{G} \longrightarrow 1,
$$

where $\underline{G} \in \mathcal{H}$ denotes the category fibred in groupoids associated to $G$.

(2) The smooth 2-group $\operatorname{Sym}_{G}(\mathcal{G})$ acts on $\mathcal{G}$, and the action covers that of $G$ on $M$.

(3) The gerbe $\mathcal{G}$ admits a $G$-equivariant structure if and only if there exists a morphism $\underline{G} \longrightarrow \operatorname{Sym}_{G}(\mathcal{G})$ of smooth 2-groups which splits the extension (1.2).

An extension similar to (1.2) was considered in [FRS16], where symmetries of a gerbe with connection were investigated in relation with higher geometric prequantisation. Infinitesimal versions of the extension (1.2) were considered in [Col11,FRS16], where it was shown that these give rise to the standard $H$-twisted Courant algebroid on $M$, where $H$ is the 3 -form curvature of the connection on $\mathcal{G}$. These considerations have been expanded on and applied to higher versions of Kaluza-Klein reductions of string theory in [Alf20].

Our point here is that in many applications, such as nonassociativity in quantum mechanics and string theory, anomalies in quantum field theory, as well as interesting topological constructions, connections on $\mathcal{G}$ only play a secondary role: in this context, they can be seen as a tool to compute the extensions (1.2) and their associated cocycles. The key to this computability is an alternative presentation of $\operatorname{Sym}_{G}(\mathcal{G})$ in terms of a categorified descent construction.

In order to work out this construction, we introduce a novel global approach to the parallel transport of a bundle gerbe. Parallel transport for gerbes has been constructed in [SW11, SW09, SW17], but for our purposes a global, rather than local, treatment is necessary. Our construction relies heavily on the transgression-regression machine for bundle gerbes [Wal16] together with the properties of the fusion product and the connection 
on the transgression line bundle that were studied in [Wal16,BW18]. Given a connection on $\mathcal{G}$, we construct its parallel transport as a quadruple $\mathrm{pt}^{\mathcal{G}}=\left(\mathrm{p} \mathrm{t}_{1}^{\mathcal{G}}, \mathrm{pt} \mathrm{G}_{2}^{\mathcal{G}}, \mathrm{pt}{ }_{\star}^{\mathcal{G}}, \varepsilon^{\mathcal{G}}\right)$, consisting of the following data: first, there is a 1-isomorphism $\mathrm{pt}_{1}^{\mathcal{G}}: \mathrm{ev}_{0}^{*} \mathcal{G} \longrightarrow \mathrm{ev}_{1}^{*} \mathcal{G}$ over the path space $P M$ of $M$, where $\mathrm{ev}_{t}: P M \longrightarrow M$ is the evaluation of a path at $t \in[0,1]$. Second, there is a 2-isomorphism $\mathrm{pt}_{2}^{\mathcal{G}}: \mathrm{pt}_{1 \mid \gamma_{0}}^{\mathcal{G}} \longrightarrow \mathrm{p} t_{1 \mid \gamma_{1}}^{\mathcal{G}}$ for every smooth homotopy with fixed endpoints between paths $\gamma_{0}$ and $\gamma_{1}$, which depends smoothly on the paths and the homotopy. The 2-isomorphisms pt $t_{\star}^{\mathcal{G}}$ and $\varepsilon^{\mathcal{G}}$ implement the compatibility of the parallel transport with concatenation of paths and with constant paths, respectively. Furthermore, the collection $\mathrm{pt}^{\mathcal{G}}$ is required to be invariant under thin homotopies in a precise way. We show

Theorem 1.3. Every bundle gerbe with connection has a canonical parallel transport.

Using the parallel transport, we are able to write down a $\mathrm{HLBdl}^{M}$-valued Čech 1cocycle on the covering of $G$ by its space of based paths. These data are equivalently transition functions for an $\mathrm{HLBdl}^{M}$-principal 2-bundle $\mathfrak{D e s} \longrightarrow \longrightarrow \underline{G}$. We construct $\mathfrak{D e s} \mathrm{L}$ explicitly by a homotopy-coherent version of the associated bundle construction. Then we prove

Theorem 1.4. The principal 2-bundle $\mathfrak{D e S} \mathrm{L} \longrightarrow \underline{G}$ is a smooth 2-group extension of $\underline{G}$ by $\mathrm{HLBdl}^{M}$. There is a weakly commutative diagram of smooth 2-groups

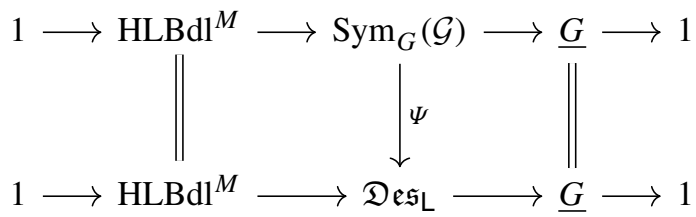

The morphism $\Psi$ is an equivalence.

In the case $M=\mathbb{R}^{d}$, where $G=\mathbb{R}_{\mathrm{t}}^{d}$ is the translation group of $\mathbb{R}^{d}$, and where $\mathcal{G}=\mathcal{I}_{B}$ is a trivial gerbe on $\mathbb{R}^{d}$ with a connection $B \in \Omega^{2}\left(\mathbb{R}^{d}\right)$ corresponding to a magnetic field, we show that the extension $\operatorname{Sym}_{\mathbb{R}_{\mathrm{t}}^{d}}(\mathcal{I}) \longrightarrow \underline{\mathbb{R}}_{\mathrm{t}}^{d}$ reproduces the 3-cocycles we obtained in [BMS19]. We achieve this by choosing a certain global section of the path fibration of $\mathbb{R}_{t}^{d}$ and implicitly pass through $\mathfrak{D e s} \mathrm{L}$ in the computation. We show that the parallel transport we defined implements nonassociative magnetic translations on the sections of the gerbe, whereas the 2-group extension $\operatorname{Sym}_{\mathbb{R}_{\mathrm{t}}^{d}}(\mathcal{I}) \longrightarrow \underline{\mathbb{R}}_{\mathrm{t}}^{d}$ allows us to understand the algebraic structure of nonassociative magnetic translations even without making any reference to sections. The latter is particularly useful in cases where there is no good notion of sections, such as when the Dixmier-Douady class of $\mathcal{G}$ is non-torsion. In particular, we study in detail the action of nonassociative magnetic translations on tori $\mathbb{T}^{d}$ and give an explicit description of $\operatorname{Sym}_{\mathbb{R}_{\mathrm{t}}^{d}}(\mathcal{G})$ for general choice of a gerbe $\mathcal{G}$ on $\mathbb{T}^{d}$.

As a further application, we show that if $\Gamma$ is a group of gauge transformations, the smooth 2-group extensions $\operatorname{Sym}_{\Gamma}(\mathcal{G}) \longrightarrow \underline{\Gamma}$ control the Faddeev-MickelssonShatashvili anomalies in quantum field theory [Fad84,FS85, Mic85]. The relation between gerbes and these anomalies has been investigated in [CM95,CM96], but only as algebraic objects, disregarding the smooth structures. The relevant bundle gerbe $\mathcal{G}$ lives on the space $\mathcal{A}$ of gauge fields and describes the obstruction to a Fock bundle descending to the orbit space $\mathcal{A} / \Gamma$. Here the extension $\operatorname{Sym}_{\Gamma}(\mathcal{G}) \longrightarrow \underline{\Gamma}$ is split, so that $\mathcal{G}$ admits 
an equivariant structure. At the same time $\mathcal{G}$ is trivialisable as a bundle gerbe, but the anomaly is precisely the obstruction to choosing a $\Gamma$-equivariant trivialisation. This allows us to understand the anomaly in a conceptual way as a higher smooth 1-cocycle on $\Gamma$.

Finally, we consider the situation where $M=G$ is a compact simply-connected Lie group, acting on itself by left multiplication, and where $\mathcal{G}$ is a bundle gerbe on $G$ whose Dixmier-Douady class generates $\mathrm{H}^{3}(G ; \mathbb{Z}) \cong \mathbb{Z}$. We motivate and propose a new smooth string 2-group model for the string group of $G$. For this, we first show that with our definition of principal 2-bundle, principal A-bundles on a manifold give rise to A-valued Čech 1-cocycles, for any smooth 2-group A. Then we call a smooth 2-group extension $\mathrm{A} \longrightarrow \mathrm{P} \longrightarrow G$ a smooth 2-group model for the string group of $G$ if $\mathrm{A}$ is equivalent to an Eilenberg-MacLane space $K(\mathbb{Z} ; 2)$ in a certain sense and the class in $\check{\mathrm{H}}^{1}(G ; \mathrm{BU}(1)) \cong \mathrm{H}^{3}(G ; \mathbb{Z})$ extracted from the 2-bundle $\mathrm{P} \longrightarrow \underline{G}$ is a generator. Using this definition of smooth string 2-group models, we show

Theorem 1.5. Let $\operatorname{Sym}_{G}(\mathcal{G})$ and $\mathfrak{D e s}$ be the smooth 2-group extensions of $\underline{G}$ by $\mathrm{HLBdl}^{G}$ constructed from $\mathcal{G}$ with respect to the left action of $G$ on itself via left multiplication. Then both $\operatorname{Sym}_{G}(\mathcal{G})$ and $\mathfrak{D} \mathfrak{e} \mathfrak{L}_{\mathrm{L}}$ are smooth 2-group models for the string group of $G$.

The remainder of this paper is organised as follows. In Sect. 2 we briefly recall some background material on diffeological spaces, bundle gerbes, and transgression. Section 3 provides a motivation of the later constructions on the level of principal bundles; many concepts become clear already at this level. In Sect. 4 we provide our definition and construction of the parallel transport associated to a bundle gerbe with connection. The construction of $\operatorname{Sym}_{G}(\mathcal{G})$ and $\mathfrak{D e s}$ takes place in Sect. 5. Here we first motivate and then introduce the necessary language of Grothendieck fibrations, smooth 2-groups, and principal 2-bundles, before defining and studying the extensions $\operatorname{Sym}_{G}(\mathcal{G})$ and $\mathfrak{D e s}$. We conclude this section by relating these extensions to equivariant structures on $\mathcal{G}$. In the remaining three sections we apply our general results: in Sect. 6 we study nonassociative magnetic translations using our parallel transport, Sect. 7 contains the discussion of chiral anomalies and the Faddeev-Mickelsson-Shatashvili anomaly, and in Sect. 8 we show that $\operatorname{Sym}_{G}(\mathcal{G})$ and $\mathfrak{D e s} L$ provide new models for the string group. We defer some technical results on categories fibred in groupoids and on principal 2-bundles to Appendix A.

\section{Preliminaries on Diffeological Spaces and Gerbes}

In this section we review some of the relevant background material related to diffeological spaces and bundle gerbes that will be used throughout this paper.

2.1. Diffeological spaces. Throughout this paper we will use diffeological spaces (see [IZ13] for an extensive introduction) to describe the smooth structure on infinitedimensional spaces such as path and mapping spaces. The idea behind diffeological spaces is to describe the smooth structure on a space $X$ by specifying the set of smooth maps from Cartesian spaces to $X$. A Cartesian space $c$ is a smooth manifold diffeomorphic to $\mathbb{R}^{n}$ for some $n \in \mathbb{N}_{0}$. We denote by Cart the category with Cartesian spaces as objects and smooth maps as morphisms.

Definition 2.1. A diffeological space is a set $X$ together with a collection of maps $c \longrightarrow$ $X$ from Cartesian spaces into $X$, called plots, such that 
(1) the composition of a plot with a smooth map between Cartesian spaces is again a plot,

(2) every map $\mathbb{R}^{0} \longrightarrow X$ is a plot, and

(3) if $f: c \longrightarrow X$ is a map such that there exists an open cover $\left\{c_{i}\right\}_{i \in I}$ of $c$ by Cartesian spaces and $f_{\mid c_{i}}$ is a plot for all $i \in I$, then $f$ is a plot.

A map $f: X \longrightarrow Y$ between diffeological spaces is smooth if it maps plots of $X$ to plots of $Y$. We denote by $\mathcal{D}$ fg the category of diffeological spaces and smooth maps. Isomorphisms in $\mathcal{D} f g$ are diffeomorphisms.

Remark 2.2. Usually plots are defined to be maps from open subsets $U$ of Cartesian spaces to $X$. Since every open subset $U$ of a Cartesian space can be covered by Cartesian spaces, both definitions are equivalent. Diffeological spaces are exactly the concrete sheaves on the site of Cartesian spaces [BH11]. This implies that the category of diffeological spaces Dfg admits all limits and colimits, and is Cartesian closed. For more background on this perspective on diffeological spaces, see also [Bun20a].

Important examples of diffeological spaces include the following.

Example 2.3. Every manifold $M$ (possibly with boundaries or corners) defines a diffeological space by declaring a map $f: c \longrightarrow M$ to be a plot if and only if $f$ is a smooth map of differentiable manifolds. This defines a fully faithful embedding of the category of smooth manifolds $\mathcal{M} f d$ into the category of diffeological spaces $\mathcal{D} f g$.

Example 2.4. Let $X$ be a diffeological space and $Y \subset X$ a subset. We can equip $Y$ with a diffeology by declaring a map $c \longrightarrow Y$ to be a plot if and only if the composition with the embedding $Y \longrightarrow X$ is a plot. This is called the subspace diffeology on $Y$.

Example 2.5. Let $X$ and $Y$ be diffeological spaces. The Cartesian product $X \times Y$ is a diffeological space by declaring a map $f: c \longrightarrow X \times Y$ to be a plot if and only if $\operatorname{pr}_{X} \circ f$ and $\operatorname{pr}_{Y} \circ f$ are plots, where $\operatorname{pr}_{X}$ and $\operatorname{pr}_{Y}$ are the respective projections of $X \times Y$ onto $X$ and $Y$. This is called the product diffeology on $X \times Y$.

Example 2.6. Let $X$ and $Y$ be diffeological spaces. The set of smooth maps $Y^{X}$ from $X$ to $Y$ becomes a diffeological space by declaring a map $f: c \longrightarrow Y^{X}$ to be a plot if and only if the map

$$
\begin{aligned}
f^{\dashv}: c \times X & \longrightarrow Y \\
(u, x) & \longmapsto f(u)(x)
\end{aligned}
$$

is smooth. This is called the mapping space diffeology on $Y^{X}$.

A smooth map $f: M \longrightarrow M^{\prime}$ between smooth manifolds is a surjective submersion if and only if it admits local sections through every point in $M$, i.e. for every point $y \in M$ there exists an open neighbourhood $U$ of $f(y)$ in $M^{\prime}$ and a smooth map $\widehat{s}: U \longrightarrow M$ such that $f \circ \widehat{s}=1_{U}$ is the identity map of $U$. Surjective submersions define a Grothendieck topology on the category of manifolds, and many (higher) geometric objects on manifolds can be constructed via sheafification with respect to this topology (see, for instance, [NS11]). On the category of diffeological spaces, a useful Grothendieck topology is induced by the subductions:

Definition 2.7. A smooth map $f: X \longrightarrow Y$ of diffeological spaces is a subduction if for all plots $\varphi: c \longrightarrow Y$ and $x \in c$ there exists an open neighbourhood $U_{x} \subset c$ of $x$ and a plot $\widehat{\varphi}_{x}: U_{x} \longrightarrow X$ such that $\varphi_{\mid U_{x}}=f \circ \widehat{\varphi}_{x}$. 
Example 2.8. Let $M$ be a connected manifold. The space of paths in $M$ with sitting instants $P M$ is the subspace of $M^{[0,1]}$ of maps which are constant in an open neighbourhood of 0 and 1 , equipped with the subspace diffeology. The evaluation maps ev $0: P M \longrightarrow M$ and $\mathrm{ev}_{1}: P M \longrightarrow M$ at 0 and 1 , respectively, are subductions.

Another source for subductions are quotient maps. Let $X$ be a diffeological space and $\sim$ an equivalence relation on $X$. Then the space $X / \sim$ becomes a diffeological space in a canonical way making the map $\pi: X \longrightarrow X / \sim$ into a subduction: a map $\varphi: c \longrightarrow X / \sim$ is a plot if and only if for all $x \in c$ there exists an open neighbourhood $U_{x} \subset c$ of $x$ and a plot $\widehat{\varphi}_{x}: U_{x} \longrightarrow X$ such that $\varphi_{\mid U_{x}}=\pi \circ \widehat{\varphi}_{x}$. Clearly all subductions are of this type for appropriate equivalence relations. Diffeological quotients behave nicely with respect to quotients of manifolds when they exist.

Proposition 2.9. Let $M$ be a manifold with a free and proper action of a Lie group $G$. Define an equivalence relation $\sim_{G}$ on $M$ by $m_{1} \sim_{G} m_{2}$ if and only if there exists $g \in G$ such that $g \cdot m_{1}=m_{2}$. Then the manifold $M / G$ and the diffeological space $M / \sim_{G}$ agree.

Proof. From [Lee13, Theorem 21.10] it follows that $\pi: M \longrightarrow M / G$ is a surjective submersion. Since every surjective submersion is a subduction, the statement follows.

Definition 2.10. Let $X$ be a diffeological space and $k \geq 0$. A $k$-form $\omega$ on $X$ consists of a family of differential forms $\omega_{\varphi} \in \Omega^{k}(c)$ indexed by the plots $\varphi: c \longrightarrow X$ of $X$ such that $\omega_{\varphi_{1}}=f^{*} \omega_{\varphi_{2}}$ for all commuting triangles

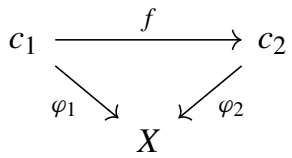

Definition 2.11 ([Wal12b, Section 3]). Let $G$ be a Lie group and $X$ a diffeological space. A principal $G$-bundle on $X$ consists of a subduction $\pi: P \longrightarrow X$ together with a fibre-preserving right action $P \times G \longrightarrow P$ such that the map

$$
\begin{aligned}
P \times G & \longrightarrow P \times_{X} P \\
(p, g) & \longmapsto(p, p \cdot g)
\end{aligned}
$$

is a diffeomorphism. A connection on a principal $G$-bundle $P$ is a 1 -form $A \in \Omega^{1}(P ; \mathfrak{g})$ satisfying

$$
\rho^{*} A=\operatorname{Ad}_{\operatorname{pr}_{G}}^{-1}\left(\operatorname{pr}_{P}^{*} A\right)+\operatorname{pr}_{G}^{*} \theta
$$

on $P \times G$, where $\rho: P \times G \longrightarrow P$ is the right $G$-action, $\theta$ is the left-invariant MaurerCartan 1-form on $G$, and $\mathrm{pr}_{P}: P \times G \longrightarrow P$ and $\mathrm{pr}_{G}: P \times G \longrightarrow G$ are the projections onto $P$ and $G$, respectively.

2.2. Bundle gerbes and transgression. Bundle gerbes are higher categorical analogues of line bundles. They provide a geometric realisation for the third cohomology group with integer coefficients. Similarly to line bundles, bundle gerbes can be equipped with connections. We briefly recall the definition of the 2-groupoid of bundle gerbes and their transgression to loop space. For details we refer to [Wal07b, Wal16, Bun17, Mur96]. 
Let $X$ be a diffeological space. We denote by $\operatorname{HLBdl}(X)\left(\operatorname{resp} . \operatorname{HLBdl}^{\nabla}(X)\right)$ the category of hermitean line bundles (resp. with connection) on $X$. Before defining bundle gerbes we need to introduce some notation: for a subduction $\pi: Y \longrightarrow X$ of diffeological spaces we denote by

$$
Y^{[n]}=\left\{\left(y_{0}, y_{1}, \ldots, y_{n-1}\right) \in Y^{\times n} \mid \pi\left(y_{0}\right)=\pi\left(y_{1}\right)=\cdots=\pi\left(y_{n-1}\right)\right\} \subset Y^{\times n}
$$

the $n$-fold iterated fibre product $Y^{[n]}=Y \times_{X} \cdots \times_{X} Y$ over $X$ equipped with the subspace diffeology. Then $Y^{[\bullet]}$ is a simplicial diffeological space corresponding to the subduction groupoid $Y \times_{X} Y \rightrightarrows Y$, and for $k<n$ and $0 \leq i_{1}<\cdots<i_{k}<n$ we define the smooth face maps

$$
\begin{aligned}
\pi_{i_{1}, \ldots, i_{k}}: Y^{[n]} & \longrightarrow Y^{[k]} \\
\left(y_{0}, y_{1}, \ldots, y_{n-1}\right) & \longmapsto\left(y_{i_{1}}, \ldots, y_{i_{k}}\right) .
\end{aligned}
$$

Definition 2.13 ([Wal16]). Let $X$ be a diffeological space. A hermitean bundle gerbe on $X$ consists of a subduction $\pi: Y \longrightarrow X$, a hermitean line bundle $L \longrightarrow Y^{[2]}$, and a unitary isomorphism $\mu: \pi_{1,2}^{*} L \otimes \pi_{0,1}^{*} L \longrightarrow \pi_{0,2}^{*} L$ of line bundles over $Y^{[3]}$, called the bundle gerbe multiplication, which is associative over $Y^{[4]}$, i.e. $\pi_{0,2,3}^{*} \mu \circ\left(\pi_{0,1,2}^{*} \mu \otimes 1\right)=$ $\pi_{0,1,3}^{*} \mu \circ\left(1 \otimes \pi_{1,2,3}^{*} \mu\right)$.

A connection on a hermitean bundle gerbe $\mathcal{G}=(\pi: Y \longrightarrow X, L, \mu)$ consists of a hermitean connection $\nabla^{L}$ on $L$ and a 2 -form $B \in \Omega^{2}(Y)$ such that

(1) the isomorphism $\mu: \pi_{1,2}^{*} L \otimes \pi_{0,1}^{*} L \longrightarrow \pi_{0,2}^{*} L$ is parallel with respect to $\nabla^{L}$, and

(2) the curvature of $\nabla^{L}$ is equal to $\mathrm{i}\left(\pi_{1}^{*} B-\pi_{0}^{*} B\right)$.

The 2-form $B$ is called a curving. The second condition implies that the closed 3-form $\mathrm{d} B=\pi^{*} H$ descends to a unique closed 3-form $H$ on $X$ with integer periods, which is called the curvature of the bundle gerbe connection $\left(\nabla^{L}, B\right)$.

Schematically, the data corresponding to a bundle gerbe can be visualised by the diagram

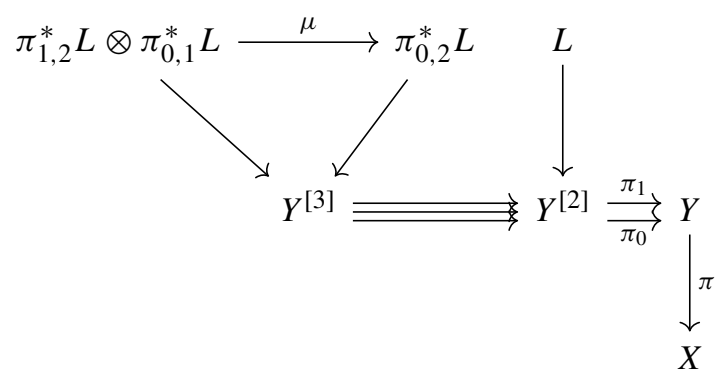

illustrating that hermitean bundle gerbes are equivalent to $\mathrm{U}(1)$-central extensions of subduction groupoids.

Example 2.14. Let $X$ be a diffeological space. The trivial hermitean bundle gerbe $\mathcal{I}$ on $X$ consists of the identity subduction $1_{X}: X \longrightarrow X$ together with the trivial hermitean line bundle $I:=X \times \mathbb{C}$ over $X^{[2]}=X$ and bundle gerbe multiplication

$$
\begin{aligned}
X \times(\mathbb{C} \otimes \mathbb{C}) & \longrightarrow X \times \mathbb{C} \\
\left(x,\left(z_{1} \otimes z_{2}\right)\right) & \longmapsto\left(x, z_{1} z_{2}\right) .
\end{aligned}
$$


For every 2-form $B \in \Omega^{2}(X)$ we can define a connection on $\mathcal{I}$ by setting $\nabla^{I}=\mathrm{d}$ and taking $B$ as the curving. We denote the resulting hermitean bundle gerbe with connection by $\mathcal{I}_{B}$. The curvature of $\mathcal{I}_{B}$ is given by $H=\mathrm{d} B$.

Hermitean bundle gerbes (resp. with connection) on a diffeological space $X$ are the objects of a symmetric monoidal bicategory which we denote by $\operatorname{BGrb}(X)$ (resp. $\left.\mathrm{BGrb}^{\nabla}(X)\right)[\mathrm{Wal07b}$.

Definition 2.15. Let $\mathcal{G}=\left(\pi: Y \longrightarrow X, L, \mu, \nabla^{L}, B\right)$ and $\mathcal{G}^{\prime}=\left(\pi^{\prime}: Y^{\prime} \longrightarrow X, L^{\prime}, \mu^{\prime}\right.$, $\left.\nabla^{L^{\prime}}, B^{\prime}\right)$ be hermitean bundle gerbes with connection on a diffeological space $X$. A 1 isomorphism $\mathcal{G} \longrightarrow \mathcal{G}^{\prime}$ of hermitean bundle gerbes (with connection) consists of a subduction $\xi: Z \longrightarrow Y \times_{X} Y^{\prime}$, a hermitean line bundle $E$ (with hermitean connection $\nabla^{E}$ ) on $Z$ and (parallel) unitary isomorphisms

$$
\alpha:\left(\left(\operatorname{pr}_{Y} \circ \xi\right)^{[2]}\right)^{*} L \otimes \xi_{1}^{*} E \longrightarrow \xi_{0}^{*} E \otimes\left(\left(\operatorname{pr}_{Y^{\prime}} \circ \xi\right)^{[2]}\right)^{*} L^{\prime}
$$

over $Z^{[2]}$ satisfying a natural set of compatibility conditions, see [Wal16] for details. We will denote such a 1 -isomorphism by $(E, \xi)\left(\right.$ resp. $\left.\left(E, \xi, \nabla^{E}\right)\right)$, or sometimes simply by E.

Remark 2.16. One can also define non-invertible 1-morphisms of bundle gerbes by using higher rank hermitean vector bundles $E$ in Definition 2.15 [Wal07b]. In that case, a 1morphism is weakly invertible if and only if the underlying hermitean vector bundle $E$ is of rank 1 [Wal07a, Proposition 2.3.4]. However, with the exception of Sect. 6, we will only consider invertible 1-morphisms of bundle gerbes in the present paper.

Definition 2.17. Let $\left(\xi_{a}: Z_{a} \longrightarrow Y \times_{X} Y^{\prime}, E_{a}, \nabla^{E_{a}}, \alpha_{a}\right)$ and $\left(\xi_{b}: Z_{b} \longrightarrow Y \times_{X}\right.$ $\left.Y^{\prime}, E_{b}, \nabla^{E_{b}}, \alpha_{b}\right)$ be 1 -isomorphisms $\mathcal{G} \longrightarrow \mathcal{G}^{\prime}$ of hermitean bundle gerbes with connection. A 2-isomorphism of bundle gerbes is an equivalence class of a subduction $\omega: W \longrightarrow Z_{a} \times_{Y \times X} Y^{\prime} Z_{b}$ and a parallel unitary isomorphism $\left(\mathrm{pr}_{Z_{a}} \circ \omega\right)^{*} E_{a} \longrightarrow$ $\left(\mathrm{pr}_{Z_{b}} \circ \omega\right)^{*} E_{b}$ satisfying a natural compatibility condition, see e.g. [Wal07b] for details and the equivalence relation.

Bundle gerbes on a diffeological space $X$ are classified by their Dixmier-Douady class in $\mathrm{H}^{3}(X ; \mathbb{Z})$, analogously to the Chern-Weil classification of line bundles by their Chern class in $\mathrm{H}^{2}(X ; \mathbb{Z})$. For a bundle gerbe with connection, the Dixmier-Douady class maps to the de Rham cohomology class of the curvature under the homomorphism $\mathrm{H}^{3}(X ; \mathbb{Z}) \longrightarrow \mathrm{H}^{3}(X ; \mathbb{R})$ induced by the inclusion of coefficient groups $\mathbb{Z} \hookrightarrow \mathbb{R}$.

Let $\mathcal{G}$ be a hermitean bundle gerbe defined over a subduction $\pi: Y \longrightarrow X$, with underlying hermitean line bundle $L \longrightarrow Y^{[2]}$. Let $\mathcal{A}: \mathcal{G} \longrightarrow \mathcal{G}$ be an endomorphism of $\mathcal{G}$, with underlying hermitean vector bundle $A$ over some subduction $\xi: Z \longrightarrow$ $Y^{[2]}$. Consider the hermitean vector bundle $L^{\vee} \otimes A$ on $Z$, where we denote the dual line bundle by $L^{\vee}$. This comes with a canonical descent isomorphism defined by the 
diagram [Wal07b, Bun17]

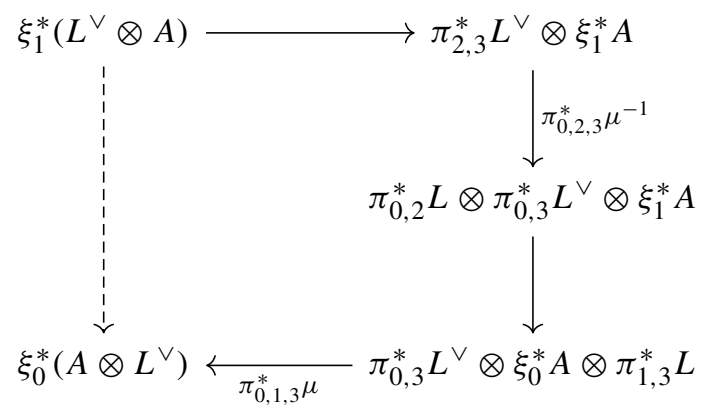

In fact, this construction establishes an equivalence of categories $\mathrm{R}: \operatorname{BGrb}(X)(\mathcal{G}, \mathcal{G}) \longrightarrow$ $\operatorname{HLBdl}(X)$.

From a hermitean bundle gerbe with connection $\mathcal{G}$ on a diffeological space $X$ we can construct the transgression line bundle $\mathcal{T} \mathcal{G}$ over the loop space $L X$ of $X$. The fibre $\mathcal{T} \mathcal{G}_{\gamma}$ over a loop $\gamma: \mathbb{S}^{1} \longrightarrow X$ consists of equivalence classes $[[\mathcal{S}], z]$ of a 2isomorphism class of a trivialisation $\mathcal{S}: \gamma^{*} \mathcal{G} \longrightarrow \mathcal{I}_{0}$ in $\mathrm{BGrb}^{\nabla}\left(\mathbb{S}^{1}\right)$ over the unit circle $\mathbb{S}^{1}$ and an element $z \in \mathbb{C}$. Two pairs $([\mathcal{S}], z)$ and $\left(\left[\mathcal{S}^{\prime}\right], z^{\prime}\right)$ are equivalent if and only if $z^{\prime}=\operatorname{hol}\left(\mathbb{S}^{1}, \operatorname{R}\left(\mathcal{S}^{\prime} \circ \mathcal{S}^{-1}\right)\right) z$. For the construction of a diffeological structure on $\mathcal{T} \mathcal{G}:=\bigsqcup_{\gamma \in L X} \mathcal{T} \mathcal{G}_{\gamma}$ we refer to [Wal16]. A connection on a line bundle over the loop space $L X$ is superficial if the holonomy around every thin loop ${ }^{1}$ is equal to 1 and thin homotopic loops ${ }^{2}$ have the same holonomy. In the situation where $X=M$ is a manifold, a superficial connection on $\mathcal{T G}$ has been constructed from the connection on $\mathcal{G}$ in [Wal16, Prop. 3.3.1]; note that in our later constructions, we will always work with bundle gerbes over manifolds. The bundle gerbe multiplication induces, for all triples of paths $\left(\gamma_{1}, \gamma_{2}, \gamma_{3}\right)$ with sitting instants and the same start and end points, a fusion product

$$
\lambda: \mathcal{T} \mathcal{G}_{\overline{\gamma_{2}} \star \gamma_{1}} \otimes \mathcal{T} \mathcal{G}_{\overline{\gamma_{3}} \star \gamma_{2}} \longrightarrow \mathcal{T} \mathcal{G}_{\overline{\gamma_{3}} \star \gamma_{1}},
$$

where $\star$ denotes the concatenation of paths and $\bar{\gamma}$ is the path $t \longmapsto \gamma(1-t)$. The fusion product depends smoothly on the paths, is parallel with respect to the superficial connection, and is associative. The connection and fusion product satisfy one further compatibility condition, related to the rotation of all loops involved by $180^{\circ}$ (see [Wal16, Definition 2.1.5]). A line bundle over $L X$ admitting all the structures discussed above is a fusion line bundle with superficial connection.

For $X=M$ a manifold, transgression extends to a functor $\mathcal{T}$ from $h B \operatorname{Grb}^{\nabla}(M)$, the 1-category obtained from $\operatorname{BGrb}^{\nabla}(M)$ by identifying isomorphic 1-morphisms, to the category of fusion line bundles with superficial connection over $L M$. The central result of [Wal16] is that $\mathcal{T}$ defines an equivalence of categories. An explicit inverse functor $\mathcal{R}$ is constructed in [Wal16] and is called regression.

\section{Group Extensions from Principal Bundles}

In this section we construct group extensions from group actions on manifolds with principal bundles. We generalise this extension to higher geometry in Sect. 5. We present

\footnotetext{
1 A loop $\Gamma \in L L X$ is thin if the adjoint map $\Gamma^{\dashv}: \mathbb{S}^{1} \times \mathbb{S}^{1} \longrightarrow X$ has at most rank 1 .

2 Two loops $\Gamma, \Gamma^{\prime} \in L L X$ are thin homotopic if there exists a homotopy $h \in P L L X$ such that the adjoint $\operatorname{map} h^{\dashv}:[0,1] \times \mathbb{S}^{1} \times \mathbb{S}^{1} \longrightarrow X$ has at most rank 2 .
} 
two perspectives on this group extension. The first one is global. The second one is local and can be formulated in terms of the parallel transport of an auxiliary connection on a principal bundle.

3.1. Global description. Let $H$ be a Lie group and $P \longrightarrow M$ a principal $H$-bundle on a manifold $M$; principal $H$-bundles on $M$ and isomorphisms form a groupoid which we denote by $\operatorname{Bun}_{H}(M)$. We consider a Lie group action

$$
\begin{aligned}
\Phi: G \times M & \longrightarrow M \\
(g, x) & \longmapsto \Phi_{g}(x)=\Phi(g, x)
\end{aligned}
$$

on the base manifold $M$, and ask whether and how this action lifts to $P$. An action of a Lie group $G$ on $M$ can equivalently be written as a smooth homomorphism of groups $\Phi: G \longrightarrow \operatorname{Diff}(M)$, where $\operatorname{Diff}(M)$ is the diffeological group of diffeomorphisms $M \longrightarrow M$. In general, the action of $G$ does not lift to $P$. Instead, we will construct a group extension

$$
1 \longrightarrow \operatorname{Gau}(P) \longrightarrow \operatorname{Sym}_{G}(P) \longrightarrow G \longrightarrow 1
$$

of $G$ by the gauge group $\operatorname{Gau}(P)$ of $P$. The group $\operatorname{Sym}_{G}(P)$ acts on the total space $P$ in a way compatible with the action of $G$ on $M$. We show that it is the universal extension of $G$ having this property.

Remark 3.1. The extension can be constructed as the pullback of the short exact sequence

$$
1 \longrightarrow \operatorname{Gau}(P) \longrightarrow \operatorname{Aut}_{G}(P) \longrightarrow \operatorname{Diff}_{P}(M) \longrightarrow 1
$$

of diffeological groups along $\Phi$, where $\operatorname{Aut}_{G}(P)$ is the group of $G$-equivariant diffeomorphisms of $P$ and $\operatorname{Diff}_{P}(M)$ is the subgroup of diffeomorphisms of $M$ which admit an equivariant lift to $P$. In the following we present a different construction which generalises directly to bundle gerbes.

We can pull back the bundle $P$ along the source and target maps of the action groupoid

$$
G \times M \underset{\mathrm{pr}_{M}}{\stackrel{\Phi}{\longrightarrow}} M
$$

We define a bundle

$$
\operatorname{Sym}_{G}(P) \stackrel{\pi}{\longrightarrow} G \quad \text { with } \operatorname{Sym}_{G}(P)_{\mid g}:=\operatorname{Bun}_{H}(M)\left(P, \Phi_{g}^{*} P\right)
$$

for all $g \in G$, where $\operatorname{Bun}_{H}(M)\left(P, \Phi_{g}^{*} P\right)$ is the collection of gauge transformations from $P$ to $\Phi_{g}^{*} P$. In order for $\operatorname{Sym}_{G}(P)$ to be a bundle over $G$, we must ensure that the fibres of $\operatorname{Sym}_{G}(P)$ are actually pairwise diffeomorphic. It might happen that a pullback bundle $\Phi_{g}^{*} P$ is no longer isomorphic to $P$ and hence the fibre over $g$ is empty. As an example, consider the action of the group $G=\mathbb{Z}$ on the 2-torus $M=\mathbb{T}^{2}$ generated by an orientation-reversing diffeomorphism $f$, and let $P \longrightarrow \mathbb{T}^{2}$ be a U(1)-bundle with nontrivial Chern class. Then $\left[f^{*} P\right]=-[P]$, and thus $\operatorname{Sym}_{\mathbb{Z}}(P)_{\mid 1}=\operatorname{Bun}_{\mathrm{U}(1)}\left(P, f^{*} P\right)=$ $\varnothing$. Hence in (3.2) we have to ensure that the fibres of $\operatorname{Sym}_{G}(P)$ are actually all nontrivial.

We restrict our attention to connected Lie groups $G$; otherwise, if $G$ is not connected, we consider only the connected component of the identity $e \in G$. We show that in this 
case the fibres are always non-trivial: we need to show that for any $g \in G$ the fibre of $\operatorname{Sym}_{G}(P) \longrightarrow G$ over $g$ is non-empty. That is, we need to show that there exists an isomorphism $P \longrightarrow \Phi_{g}^{*} P$ of $H$-bundles over $M$. Let $f_{P}: M \longrightarrow \mathrm{B} H$ be a map that classifies the bundle $P \longrightarrow M$. Then $\Phi_{g}^{*} P$ is classified by the map $f_{P} \circ \Phi_{g}: M \longrightarrow \mathrm{B} H$. Since $G$ is connected, we can find a smooth path $\gamma:[0,1] \longrightarrow G$ with $\gamma(0)=e$ and $\gamma(1)=g$. Consider the smooth map

$$
\begin{aligned}
\Phi_{\gamma}:[0,1] \times M & \longrightarrow M \\
(t, x) & \longmapsto \Phi_{\gamma(t)}(x) .
\end{aligned}
$$

We can postcompose this map by $f_{P}$ to obtain a homotopy

$$
f_{P} \circ \Phi_{\gamma}:[0,1] \times M \longrightarrow \mathrm{B} H
$$

from $f_{P}$ to $f_{P} \circ \Phi_{g}$. This shows that there exists a bundle isomorphism $P \longrightarrow \Phi_{g}^{*} P$. We note for later use that this argument generalises to $n$-gerbes $\mathcal{G}$, as these are classified by maps $f_{\mathcal{G}}: M \longrightarrow \mathrm{B}^{n+1} \mathrm{U}(1)$.

In order to equip the set $\operatorname{Sym}_{G}(P)$ with a diffeology, we note that $\operatorname{Sym}_{G}(P)$ can be identified with the subspace of the Cartesian product of the space of $H$-equivariant diffeomorphisms $P \longrightarrow P$ which cover the action of an arbitrary element $g \in G$ on $M$ with $G$, and equip $\operatorname{Sym}_{G}(P)$ with the subspace diffeology. Concretely, for $c \in \mathcal{C}$ art, a map $f: c \longrightarrow \operatorname{Sym}_{G}(P)$ is a plot if and only if the composition $\pi \circ f: c \longrightarrow G$ is smooth and the induced map $\operatorname{pr}_{M}^{*} P \longrightarrow \Phi_{f}^{*} P$ is an isomorphism in $\operatorname{Bun}_{H}(c \times M)$, where $\operatorname{pr}_{M}: c \times M \longrightarrow M$ is the projection onto $M$ and $\Phi_{f}=\Phi \circ\left(f \times 1_{M}\right)$. The automorphism group or group of gauge transformations

$$
\operatorname{Gau}(P):=\operatorname{Bun}_{H}(M)(P, P)
$$

acts simply and transitively on each fibre $\operatorname{Sym}_{G}(P)_{\mid g}$ from the right via precomposition. The set $\operatorname{Gau}(P)$ forms a diffeological group with respect to the composition of automorphisms and the smooth structure induced from the mapping space diffeology on $P^{P}$.

Proposition 3.3. $\pi: \operatorname{Sym}_{G}(P) \longrightarrow G$ is a principal Gau(P)-bundle on $G$.

Proof. We verify that the map $\pi: \operatorname{Sym}_{G}(P) \longrightarrow G$ is a subduction. Let $f: c \longrightarrow G$ be a plot. We can pick an isomorphism $\varphi_{f}: \operatorname{pr}_{M}^{*} P \longrightarrow \Phi_{f}^{*} P$ (since $c$ is contractible) and define the map

$$
\begin{aligned}
\widehat{f}: c & \longrightarrow \operatorname{Sym}_{G}(P) \\
x & \longmapsto \varphi_{f \mid\{x\} \times M} .
\end{aligned}
$$

The map $\widehat{f}$ is a smooth lift of the plot $f$, showing that $\operatorname{Sym}_{G}(P) \longrightarrow G$ is a subduction.

The map

$$
\begin{aligned}
\operatorname{Sym}_{G}(P) \times{ }_{G} \operatorname{Sym}_{G}(P) & \longrightarrow \operatorname{Sym}_{G}(P) \times \operatorname{Gau}(P) \\
\left(\varphi: P \longrightarrow \Phi_{g}^{*} P, \varphi^{\prime}: P \longrightarrow \Phi_{g}^{*} P\right) & \longmapsto\left(\varphi, \varphi^{-1} \circ \varphi^{\prime}\right)
\end{aligned}
$$

provides a smooth inverse to the $\operatorname{map}_{\operatorname{Sym}}(P) \times \operatorname{Gau}(P) \longrightarrow \operatorname{Sym}_{G}(P) \times{ }_{G} \operatorname{Sym}_{G}(P)$ from (2.12), and the result follows. 
Proposition 3.4. $\operatorname{Sym}_{G}(P)$ is a diffeological group. The principal bundle $\operatorname{Sym}_{G}(P) \longrightarrow$ $G$ is part of an extension of diffeological groups

$$
1 \longrightarrow \operatorname{Gau}(P) \longrightarrow \operatorname{Sym}_{G}(P) \longrightarrow G \longrightarrow 1
$$

Proof. To complete the proof we need to equip $\operatorname{Sym}_{G}(P)$ with a diffeological group structure such that the map $\operatorname{Sym}_{G}(P) \longrightarrow G$ becomes a morphism of diffeological groups. Consider isomorphisms $\psi: P \longrightarrow \Phi_{g}^{*} P$ and $\phi: P \longrightarrow \Phi_{g^{\prime}}^{*} P$ for $g, g^{\prime} \in G$. We set

$$
\mu(\psi, \phi):=\Phi_{g^{\prime}}^{*} \psi \circ \phi: P \longrightarrow \Phi_{g^{\prime}}^{*} P \longrightarrow \Phi_{g^{\prime}}^{*} \Phi_{g}^{*} P=\Phi_{g g^{\prime}}^{*} P
$$

This is associative by the associativity of pullbacks, the multiplication in $G$, and composition of morphisms. The inverse of an element $\psi: P \longrightarrow \Phi_{g}^{*} P$ with respect to $\mu$ is the isomorphism

$$
P=\Phi_{g^{-1}}^{*} \Phi_{g}^{*} P \stackrel{\Phi_{g^{-1}}^{*} \psi^{-1}}{\longrightarrow} \Phi_{g^{-1}}^{*} P,
$$

and the result follows from the observation that these maps are smooth.

Proposition 3.5. The group $\operatorname{Sym}_{G}(P)$ acts smoothly on $P$, lifting the action of $G$ on $M$. It is universal in the following sense: let $\widehat{G}$ be a Lie group, $\varphi: \widehat{G} \longrightarrow G$ a Lie group homomorphism and $\widehat{\psi}: \widehat{G} \times P \longrightarrow P$ an action of $\widehat{G}$ on $P$ making the diagram

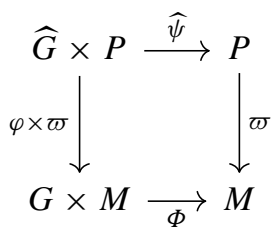

commute, where $\varpi: P \longrightarrow M$ is the bundle projection. Then there exists a unique smooth group homomorphism $\widehat{G} \longrightarrow \operatorname{Sym}_{G}(P)$ such that the diagram

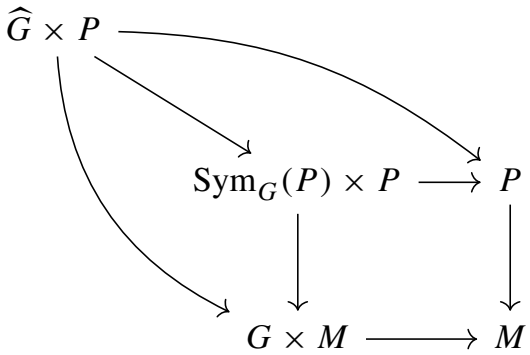

commutes.

Proof. The action is via the evaluation

$$
\begin{aligned}
\widehat{\Phi}: \operatorname{Sym}_{G}(P) \times P & \longrightarrow P \\
(\phi, p) & \longmapsto \phi(p)=\phi_{\mid \varpi(p)}(p) .
\end{aligned}
$$


The unique smooth group homomorphism in the universality statement is

$$
\begin{aligned}
& \widehat{G} \longrightarrow \operatorname{Sym}_{G}(P) \\
& \widehat{g} \longmapsto\left(\widehat{\psi}_{\widehat{g}}: P \longrightarrow \Phi_{\varphi(\widehat{g})}^{*} P\right),
\end{aligned}
$$

and the result follows.

The construction of the group $\operatorname{Sym}_{G}(P)$ is functorial in $P$, i.e. an isomorphism of bundles $\psi: P \longrightarrow P^{\prime}$ induces an isomorphism of group extensions

$$
\begin{aligned}
\widehat{\psi}: \operatorname{Sym}_{G}(P) \longrightarrow \operatorname{Sym}_{G}\left(P^{\prime}\right) \\
\left(f: P \longrightarrow g^{*} P\right) \longmapsto\left(P^{\prime} \stackrel{\psi^{-1}}{\longrightarrow} P \stackrel{f}{\longrightarrow} g^{*} P \stackrel{g^{*} \psi}{\longrightarrow} g^{*} P^{\prime}\right) .
\end{aligned}
$$

3.2. Equivariant bundles. Let $G$ be a connected Lie group, $M$ a manifold with $G$-action $\Phi: G \times M \longrightarrow M$, and $P$ a principal $H$-bundle over $M$. A $G$-equivariant structure on $P$ consists of an isomorphism $\chi: \operatorname{pr}_{M}^{*} P \longrightarrow \Phi^{*} P$ of principal bundles over $G \times M$ such that the diagram

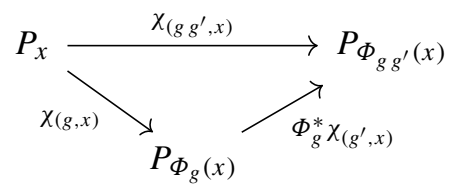

commutes for all $g, g^{\prime} \in G$ and $x \in M$. We denote by $\mathcal{E}(P)$ the set of equivariant structures on $P$. A splitting $s$ of $\pi: \operatorname{Sym}_{G}(P) \longrightarrow G$ is a smooth group homomorphism $s: G \longrightarrow \operatorname{Sym}_{G}(P)$ such that $\pi \circ s=1_{G}$. We denote the set of splittings of $\pi: \operatorname{Sym}_{G}(P) \longrightarrow G$ by $\mathcal{S}\left(G ; \operatorname{Sym}_{G}(P)\right)$.

Proposition 3.7. There is a natural bijection of sets $\Xi: \mathcal{E}(P) \longrightarrow \mathcal{S}\left(G ; \operatorname{Sym}_{G}(P)\right)$. In particular, the bundle $P$ admits an equivariant structure if and only if the extension

$$
1 \longrightarrow \operatorname{Gau}(P) \longrightarrow \operatorname{Sym}_{G}(P) \longrightarrow G \longrightarrow 1
$$

is trivial as an extension of diffeological groups.

Proof. Let $(P, \chi)$ be an equivariant bundle. We define $\Xi(P, \chi)(g): P \longrightarrow \Phi_{g}^{*} P$ to be $\chi \mid\{g\} \times M$. The inverse $\Xi^{-1}: \mathcal{S}\left(G ; \operatorname{Sym}_{G}(P)\right) \longrightarrow \mathcal{E}(P)$ can be constructed by sending a splitting $s: G \longrightarrow \operatorname{Sym}_{G}(P)$ to the isomorphism $\Xi^{-1}(s): \operatorname{pr}_{M}^{*} P \longrightarrow \Phi^{*} P$ which is given by $s(g)(x): P_{x} \longrightarrow P_{\Phi_{g}(x)}$ at $(g, x) \in G \times M$.

Let $(P, \chi)$ and $\left(P^{\prime}, \chi^{\prime}\right)$ be $G$-equivariant $H$-bundles on $M$. An isomorphism $\psi: P \longrightarrow$ $P^{\prime}$ is equivariant if the diagram

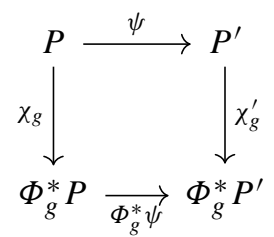


commutes for all $g \in G$. The equivariant structures on $P$ and $P^{\prime}$ can be described by smooth group homomorphisms $s_{P}: G \longrightarrow \operatorname{Sym}_{G}(P)$ and $s_{P^{\prime}}: G \longrightarrow \operatorname{Sym}_{G}\left(P^{\prime}\right)$. Since the isomorphism $\widehat{\psi}$ defined in (3.6) intertwines the action of $\operatorname{Sym}_{G}(P)$ and $\operatorname{Sym}_{G}\left(P^{\prime}\right)$ on $P$ and $P^{\prime}$, respectively, it follows that $\psi$ is equivariant if and only if $s_{P^{\prime}}=\widehat{\psi} \circ s_{P}$. Hence the smooth group extension $\operatorname{Sym}_{G}$ contains all information on equivariance.

3.3. Description via parallel transport. The extension $\operatorname{Sym}_{G}(P)$ can be described more explicitly using the parallel transport of a connection on $P$, as we will now explain. In Sect. 6 we apply this to the description of magnetic translations in quantum mechanics. We consider a principal $H$-bundle $P \longrightarrow M$. Let $P_{0} G$ denote the diffeological space of smooth paths in $G$ with sitting instants based at $e \in G$, ev $: P_{0} G \longrightarrow G$ the evaluation at the end point, $\left(P_{0} G\right)^{[2]}$ the fibre product $P_{0} G \times_{G} P_{0} G$ with respect to ev 1 , and $L M$ the space of smooth loops in $M$. We denote by $\star$ the concatenation of paths. For a path $\gamma:[0,1] \longrightarrow G$ we denote by $\bar{\gamma}$ the precomposition of $\gamma$ with

$$
\begin{aligned}
{[0,1] } & \longrightarrow[0,1] \\
t & \longmapsto 1-t .
\end{aligned}
$$

For a path $\gamma \in P_{0} G$ and a point $x \in M$, set

$$
\begin{aligned}
\gamma_{x}:[0,1] & \longrightarrow M \\
t & \longmapsto \Phi_{\gamma(t)}(x) .
\end{aligned}
$$

Endow $P$ with an arbitrary connection $A$. The $H$-bundle $P$ with connection then induces a principal $\operatorname{Gau}(P)$-bundle on $G$ as follows: we set

$$
\mathcal{L}_{G}:=\left(P_{0} G \times \operatorname{Gau}(P)\right) / \sim,
$$

where we define the equivalence relation

$$
\begin{aligned}
& (\gamma, \phi) \sim(\alpha, \operatorname{hol}(P, \alpha, \gamma) \circ \phi) \\
& \text { with } \operatorname{hol}(P, \alpha, \gamma)(x):=\operatorname{hol}\left(P,(\bar{\alpha} \star \gamma)_{x}\right) \in \operatorname{End}\left(P_{x}\right)
\end{aligned}
$$

for all $(\gamma, \alpha) \in\left(P_{0} G\right)^{[2]}$ and $x \in M$, and we interpret the holonomy of $P$ along a loop starting and ending at $x$ as an endomorphism of the fibre $P_{x}$. Note that, with this notation, we have defined a smooth map hol $(P,-):\left(P_{0} G\right)^{[2]} \longrightarrow \operatorname{Gau}(P)$. We endow $\mathcal{L}_{G}$ with the quotient diffeology.

Then the $\operatorname{Gau}(P)$-bundle $\mathcal{L}_{G} \longrightarrow G$ can be defined in terms of descent data as follows: the action $\Phi$ of $G$ on $M$ induces a smooth map

$$
\begin{aligned}
L \Phi:\left(P_{0} G\right)^{[2]} \times M & \longrightarrow L M \\
(\gamma, \alpha, x) & \longmapsto(\bar{\alpha} \star \gamma)_{x} .
\end{aligned}
$$

Explicitly,

$$
(\bar{\alpha} \star \gamma)_{x}(t)=\Phi_{(\bar{\alpha} \star \gamma)(t)}(x) \in M
$$

for all $t \in[0,1]$ and $x \in M$. The descent data for the bundle $\mathcal{L}_{G}$ consists of the subduction $P_{0} G \longrightarrow G$, the trivial bundle $P_{0} G \times \mathrm{Gau}(P) \longrightarrow P_{0} G$, and the smooth map

$$
\begin{aligned}
\mathrm{g}:\left(P_{0} G\right)^{[2]} & \longrightarrow \mathrm{Gau}(P) \\
(\gamma, \alpha) & \longmapsto \mathrm{g}(\gamma, \alpha)=\operatorname{hol}(P, \bar{\alpha} \star \gamma) .
\end{aligned}
$$


Proposition 3.9. The total space $\mathcal{L}_{G}$ is a smooth group extension

$$
1 \longrightarrow \operatorname{Gau}(P) \longrightarrow \mathcal{L}_{G} \longrightarrow G \longrightarrow 1
$$

Proof. Let $\gamma$ and $\gamma^{\prime}$ be two paths in $G$. The evaluation ev $e_{1}: P_{0} G \longrightarrow G$ is a group homomorphism with respect to the pointwise product of paths.

Let $x \in M$ be an arbitrary point. To any triple $\left(x, \gamma, \gamma^{\prime}\right)$, we can associate a map

$$
\begin{aligned}
\Delta^{2}\left(x, \gamma, \gamma^{\prime}\right):\left|\Delta^{2}\right| & \longrightarrow M \\
\left(t_{1}, t_{2}\right) & \longmapsto \Phi_{\gamma\left(t_{1}\right)}\left(\gamma_{x}^{\prime}\left(t_{2}\right)\right),
\end{aligned}
$$

where $\left|\Delta^{2}\right|$ is the standard topological 2-simplex with $\left|\Delta^{2}\right| \cong\left\{\left(t_{1}, t_{2}\right) \in \mathbb{R}^{2} \mid 0 \leq t_{2} \leq\right.$ $\left.t_{1} \leq 1\right\}$. Diagrammatically, this is a homotopy

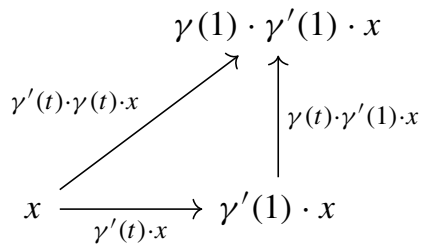

between the product path $\gamma \gamma^{\prime} \in P_{0} G$ and the concatenated path $\left(\gamma \gamma^{\prime}(1)\right) \star \gamma^{\prime} \in P_{0} G$. For $\gamma, \gamma^{\prime} \in P_{0} G$ and $\phi, \phi^{\prime} \in \operatorname{Gau}(P)$, we define

$$
\mu\left((\gamma, \phi),\left(\gamma^{\prime}, \phi^{\prime}\right)\right):=\left(\gamma \gamma^{\prime}, \mathrm{pt}_{\gamma \gamma^{\prime}}^{-1} \circ \mathrm{pt}_{\gamma\left(\gamma^{\prime}(1)\right)} \circ\left(\Phi_{\gamma^{\prime}(1)}^{*} \phi\right) \circ \mathrm{pt}_{\gamma^{\prime}} \circ \phi^{\prime}\right),
$$

where we denote by $\mathrm{pt}_{\gamma}$ the isomorphism $P \longrightarrow \Phi_{\gamma_{(1)}^{*}}^{*} P$ defined at a point $x \in M$ by the parallel transport along the path $\gamma_{x}$. This is well-defined: let $\alpha, \alpha^{\prime} \in P_{0} G$ with $\gamma(1)=\alpha(1)$ and $\gamma^{\prime}(1)=\alpha^{\prime}(1)$. Then

$$
\begin{aligned}
& \mu\left((\alpha, \operatorname{hol}(P, \alpha, \gamma) \circ \phi),\left(\alpha^{\prime}, \operatorname{hol}\left(P, \alpha^{\prime}, \gamma^{\prime}\right) \circ \phi^{\prime}\right)\right) \\
& \quad=\left(\alpha \alpha^{\prime}, \mathrm{pt}_{\alpha \alpha^{\prime}}^{-1} \circ \Phi_{\alpha^{\prime}(1)}^{*}\left(\mathrm{pt}_{\alpha} \circ \operatorname{hol}(P, \alpha, \gamma) \circ \phi^{\prime}\right) \circ \mathrm{pt}_{\alpha^{\prime}} \circ \operatorname{hol}\left(P, \alpha^{\prime}, \gamma^{\prime}\right) \circ \phi^{\prime}\right) \\
& \quad=\left(\alpha \alpha^{\prime}, \operatorname{hol}\left(P, \alpha \alpha^{\prime}, \gamma \gamma^{\prime}\right) \circ \mathrm{pt}_{\gamma \gamma^{\prime}}^{-1} \circ \Phi_{\gamma^{\prime}(1)}^{*}\left(\mathrm{pt}_{\gamma} \circ \phi^{\prime}\right) \circ \mathrm{pt}_{\gamma^{\prime}} \circ \phi^{\prime}\right) \\
& \quad=\left(\gamma \gamma^{\prime}, \mathrm{pt}_{\gamma \gamma^{\prime}}^{-1} \circ \Phi_{\gamma^{\prime}(1)}^{*}\left(\mathrm{pt}_{\gamma} \circ \phi^{\prime}\right) \circ \mathrm{pt}_{\gamma^{\prime}} \circ \phi^{\prime}\right) \\
& \quad=\mu\left((\gamma, \phi),\left(\gamma^{\prime}, \phi^{\prime}\right)\right),
\end{aligned}
$$

where we used $\Phi_{\gamma^{\prime}(1)}^{*} \mathrm{pt} t_{\gamma}=\mathrm{pt}_{\gamma\left(\gamma^{\prime}(1)\right)}$. Associativity then follows immediately from the associativity of the products in $P_{0} G$ and $\operatorname{Gau}(P)$, together with associativity of taking pullbacks. Smoothness follows from the definition of the quotient diffeology and the smooth dependence of parallel transport on the path.

Remark 3.11. For abelian structure group $H$, we can use the fact that parallel transport commutes with gauge transformations to get the simplified expression

$$
\mu\left((\gamma, \phi),\left(\gamma^{\prime}, \phi^{\prime}\right)\right)=\left(\gamma \gamma^{\prime}, \operatorname{hol}\left(P, \partial\left|\Delta^{2}\right|\right)\left(\Phi_{\gamma^{\prime}(1)}^{*} \phi\right) \circ \phi^{\prime}\right)
$$

for the multiplication (3.10). 
Remark 3.12. If $G$ is abelian, then the multiplicative structure yields isomorphisms

$$
\mathcal{L}_{G \mid g} \times \mathcal{L}_{G \mid g^{\prime}} \longrightarrow \mathcal{L}_{G \mid g g^{\prime}}=\mathcal{L}_{G \mid g^{\prime} g} \longrightarrow \mathcal{L}_{G \mid g^{\prime}} \times \mathcal{L}_{G \mid g}
$$

for all $g, g^{\prime} \in G$. That is, the group extension $\mathcal{L}_{G}$ spoils the commutativity of $G$, since its fibres multiply commutatively only up to coherent isomorphism.

We summarise the connection to the construction from Sect. 3.1 in

Proposition 3.13. Let $G$ be a connected Lie group, and let $P \longrightarrow M$ be a principal $H$-bundle on a manifold $M$ with smooth $G$-action. The map

$$
\begin{aligned}
\Gamma: \mathcal{L}_{G} & \longrightarrow \operatorname{Sym}_{G}(P) \\
{[(\gamma, \phi)] } & \longmapsto\left(\mathrm{pt}_{\gamma} \circ \phi: P \longrightarrow \Phi_{\gamma(1)}^{*} P\right)
\end{aligned}
$$

is an isomorphism of diffeological group extensions of $G$.

Proof. The map is well-defined: consider two representatives $(\gamma, \phi)$ and $(\alpha, \operatorname{hol}(P, \alpha, \gamma) \circ$ $\phi$ ) of the same equivalence class in $\mathcal{L}_{G}$, and calculate

$$
\mathrm{p} t_{\alpha} \circ \operatorname{hol}(P, \alpha, \gamma) \circ \phi=\mathrm{pt}_{\alpha} \circ \mathrm{pt} \mathrm{t}_{\bar{\alpha}} \circ \mathrm{p} t_{\gamma} \circ \phi=\mathrm{p} t_{\gamma} \circ \phi
$$

The map is bijective, because two gauge transformations $P \longrightarrow \Phi_{g}^{*} P$ differ by exactly one gauge transformation of $P$. It also follows directly from the definition that $\Gamma$ is a morphism of extensions. We check that $\Gamma$ is a group homomorphism: for $[(\gamma, \phi)],\left[\left(\gamma^{\prime}, \phi^{\prime}\right)\right] \in$ $\mathcal{L}_{G}$ we compute

$$
\begin{aligned}
\mu\left(\Gamma(\gamma, \phi), \Gamma\left(\gamma^{\prime}, \phi^{\prime}\right)\right) & =\mu\left(\mathrm{pt}_{\gamma} \circ \phi, \mathrm{pt}_{\gamma^{\prime}} \circ \phi^{\prime}\right) \\
& =\Phi_{\gamma^{\prime}(1)}^{*}\left(\mathrm{pt}_{\gamma} \circ \phi\right) \circ \mathrm{pt}_{\gamma^{\prime}} \circ \phi^{\prime} \\
& =\Gamma\left(\gamma \gamma^{\prime}, \mathrm{pt}_{\gamma \gamma^{\prime}}^{-1} \circ \mathrm{pt}_{\gamma\left(\gamma^{\prime}(1)\right)} \circ\left(\Phi_{\gamma^{\prime}(1)}^{*} \phi\right) \circ \mathrm{pt}_{\gamma^{\prime}} \circ \phi^{\prime}\right) \\
& =\Gamma\left(\mu\left((\gamma, \phi),\left(\gamma^{\prime}, \phi\right)\right)\right) .
\end{aligned}
$$

Finally, we verify that $\Gamma$ is smooth. Let $f: c \longrightarrow \mathcal{L}_{G}$ be a plot admitting a lift $\widehat{f}: c \longrightarrow P_{0} G \times \operatorname{Gau}(P)$. We denote the components of $\widehat{f}$ by $\widehat{f}_{\gamma}$ and $\widehat{f}_{\mathrm{Gau}}(P)$. It is enough to show that

$$
\begin{aligned}
M^{*} P & \longrightarrow \Phi_{\hat{f}_{\gamma}}^{*} P \\
P_{x} \ni p & \longmapsto \operatorname{pt}_{\widehat{f}_{\gamma}(u)}\left(\widehat{f}_{\operatorname{Gau}(P)}(u)(p)\right) \in P_{\Phi_{\widehat{f}_{\gamma}(u)(1)}(x)}
\end{aligned}
$$

is a gauge transformation. This follows from the smoothness of parallel transport (recalled in Sect. 4.1 below).

Corollary 3.14. The action $\Phi: G \times M \longrightarrow M$ lifts to an action

$$
\widehat{\Phi}: \mathcal{L}_{G} \times P \longrightarrow P
$$

which covers the action of $G$ on $M$. 


\section{A Global Approach to Parallel Transport for Bundle Gerbes}

In Sect. 3 we have constructed two diffeological groups, $\operatorname{Sym}_{G}(P)$ and $\mathcal{L}_{G}$, which extend $G$ and control the existence of $G$-equivariant structures on a principal bundle $P$ over $M$. The key to constructing $\mathcal{L}_{G}$, as well as to comparing the groups $\operatorname{Sym}_{G}(P)$ and $\mathcal{L}_{G}$ (see Sect. 3.3), was the parallel transport on the principal bundle $P$.

If one replaces the principal bundle $P$ by a bundle gerbe $\mathcal{G}$ on $M$, there exist categorified versions of both these constructions which will be given in Sect. 5. However, in order to write down the categorification of $\mathcal{L}_{G}$ we need a notion of parallel transport for $\mathcal{G}$. In this section we give a definition of parallel transport for $\mathcal{G}$ suited for our purposes and explicitly construct such a parallel transport from any connection on $\mathcal{G}$. Our construction relies heavily on Waldorf's transgression-regression machine [Wal16].

There is a different approach to the parallel transport on a bundle gerbe developed by Schreiber and Waldorf [SW09,SW11,SW17]. It relies on their technology of transport functors and is based on local constructions, which are then glued to global objects. In [Wal18], this has been extended to a canonical assignment of a parallel transport (in terms of a transport 2-functor) to any principal 2-bundle with connection whose structure group is a Lie 2-group.

Here, in contrast, we directly define and construct a global version of parallel transport suitable for our purposes. As our main goal in this paper is the construction of categorified smooth group extensions, we leave it for future work to prove in detail that our notion of parallel transport for $\mathcal{G}$ agrees with that of Schreiber and Waldorf, and instead focus on building the necessary input for the constructions in Sect. 5.

4.1. A path space approach to parallel transport on line bundles. Before we give our definition and construction of the parallel transport for bundle gerbes, we recast the parallel transport on line bundles from a global perspective. Our notion of parallel transport for bundle gerbes will then be a categorification of this picture. Let $M$ be a connected smooth manifold, and fix a base point $x \in M$; if $M$ is not connected, we restrict to its connected components individually. We denote by $P M$ the diffeological space of smooth paths with sitting instants in $M$ and by $P_{0} M$ the subspace of paths starting at $x$. Let $L$ be a line bundle on $M$ with connection. The smoothness of the parallel transport on $L$ can be encoded as follows: for $t \in[0,1]$, denote by ev $t: P M \longrightarrow M, \gamma \longmapsto \gamma(t)$, the evaluation at $t$. Parallel transport on $L$ is in particular an isomorphism

$$
\begin{gathered}
\mathrm{pt}^{L}: \mathrm{ev}_{0}^{*} L \longrightarrow \mathrm{ev}_{1}^{*} L \\
L_{\gamma(0)}=\mathrm{ev}_{0}^{*} L_{\gamma} \ni \ell \longmapsto \mathrm{pt}_{\gamma}^{L}(\ell) \in L_{\gamma(1)}=\mathrm{ev}_{1}^{*} L_{\gamma}
\end{gathered}
$$

of line bundles over $P M$.

There is a different way to construct this isomorphism using descent. Via transgression and regression [Wal12b] we can construct a bundle $\mathcal{R} \mathcal{T}(L)$, which is isomorphic to $L$, 
from the descent data

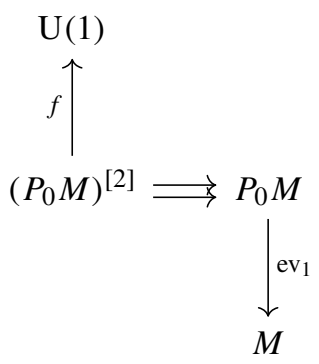

with respect to the path fibration. Here $f$ is constructed as in Sect. 3.3 from the holonomy of $L$. The total space of the line bundle $\mathcal{R} \mathcal{T}(L)$ consists of equivalence classes of pairs $(\gamma, \zeta) \in P_{0} M \times \mathbb{C}$, where the equivalence relation reads as $\left(\gamma_{1}, \zeta\right) \sim\left(\gamma_{2}, f\left(\gamma_{1}, \gamma_{2}\right) \zeta\right)$ for $\left(\gamma_{1}, \gamma_{2}\right) \in\left(P_{0} M\right)^{[2]}$ and $\zeta \in \mathbb{C}$. An isomorphism $g_{\chi}: \mathcal{R} \mathcal{T}(L) \longrightarrow L$ can be constructed by picking a trivialisation $\chi: \mathbb{C} \longrightarrow L_{x}$ of the fibre of $L$ over the base point $x \in M$ and defining

$$
g_{\chi}([\gamma, \zeta]):=\mathrm{pt}_{\gamma}^{L}(\chi(\zeta)) .
$$

The pullbacks $\operatorname{ev}_{0}^{*} \mathcal{R} \mathcal{T}(L)$ and $\operatorname{ev}_{1}^{*} \mathcal{R} \mathcal{T}(L)$ are thus described in terms of descent data with respect to the covers $\operatorname{ev}_{0}^{*} P_{0} M \cong P_{0} M \times_{M} P M \longrightarrow M$ and $\operatorname{ev}_{1}^{*} P_{0} M \cong$ $P M \times{ }_{M} P_{0} M \longrightarrow M$, respectively. In order to construct the isomorphism pt ${ }^{\mathcal{T}(L)}$ explicitly we use the space (see Fig. 1)

$$
P_{\partial \Delta^{2}} M:=\mathrm{ev}_{0}^{*} P_{0} M \times P M \mathrm{ev}_{1}^{*} P_{0} M \cong P_{0} M \times_{M} P M \times_{M} P_{0} M,
$$

which fits into the diagram

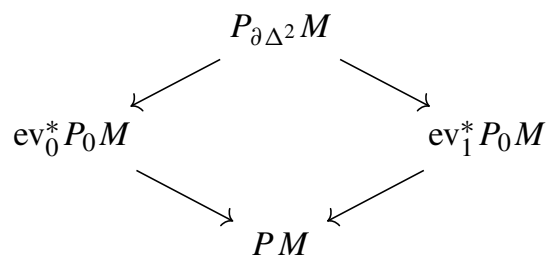

An isomorphism from $\mathrm{ev}_{0}^{*} \mathcal{R} \mathcal{T}(L)$ to $\mathrm{ev}_{1}^{*} \mathcal{R} \mathcal{T}(L)$ can be described by a function $P_{\partial \Delta^{2}} M \longrightarrow \mathrm{U}(1)$ which is compatible with the descent data. There is a canonical choice for such a function given by

$$
P_{\partial \Delta^{2}} M \longrightarrow L M \stackrel{\text { hol }}{\longrightarrow} \mathrm{U}(1) .
$$

Concretely, the induced map is

$$
\begin{aligned}
& \mathrm{pt}^{\mathcal{R} \mathcal{T}(L)}: \mathrm{ev}_{0}^{*} \mathcal{R} \mathcal{T}(L) \longrightarrow \mathrm{ev}_{1}^{*} \mathcal{R} \mathcal{T}(L)
\end{aligned}
$$

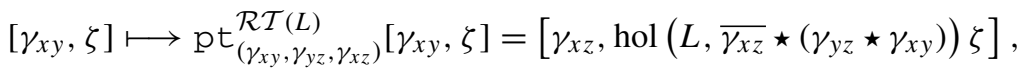

where $x$ is the fixed base point of $M$ while $y, z \in M$ are arbitrary points, and $\gamma_{a b}$ denotes a path from $a$ to $b$ for $a, b \in\{x, y, z\}$. The holonomy appearing here agrees 

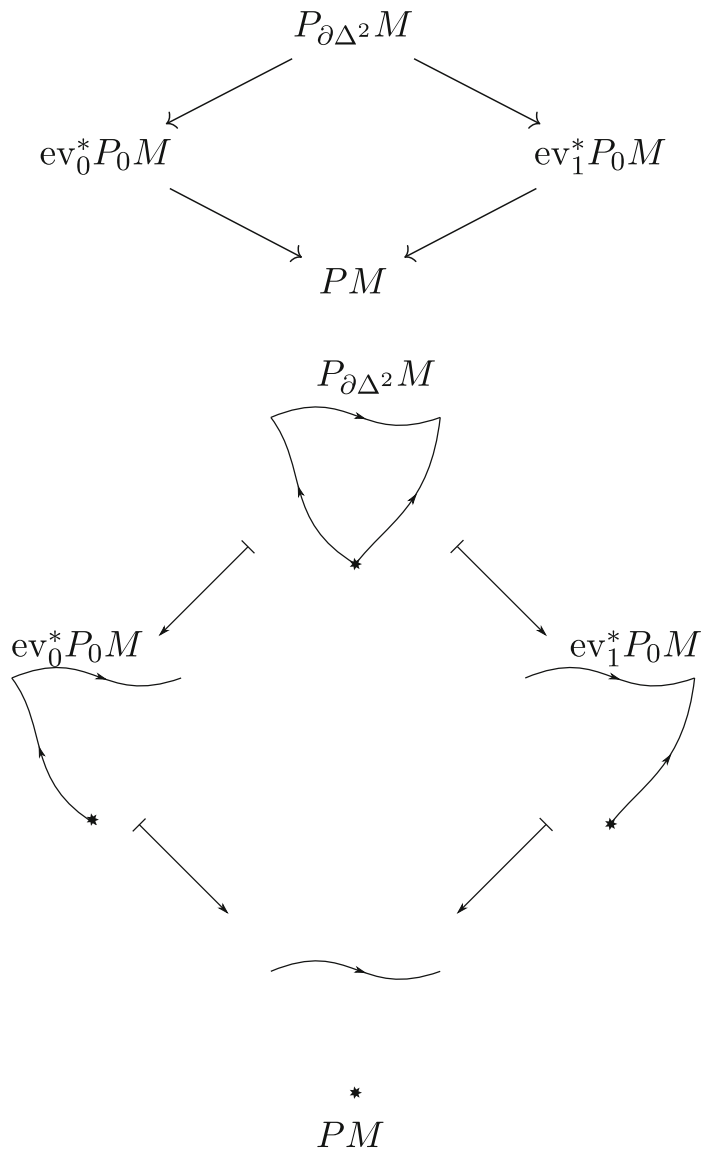

Fig. 1. Elements in the spaces $P_{\partial \Delta^{2}} M$, ev ${ }_{0}^{*} P_{0} M$ and ev ${ }_{1}^{*} P_{0} M$

with $\operatorname{hol}\left(L,\left(\overline{\gamma_{x z}} \star \gamma_{y z}\right) \star \gamma_{x y}\right)$. The construction is independent of all choices involved. Now a straightforward computation shows that the diagram

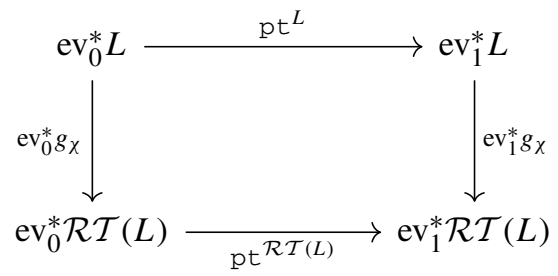

commutes. This shows that we can construct the parallel transport on $L$ completely in terms of the descent data with respect to the path fibration. For bundle gerbes, the analogue of $\mathrm{pt}^{L}$ is difficult to define directly, but an analogous approach via descent data on $P_{0} M$ allows us to solve this problem. 
4.2. Global definition of parallel transport on bundle gerbes. As before, let $M$ be a manifold, and let $\mathcal{G} \in \operatorname{BGrb}(M)$ be a bundle gerbe on $M$. A parallel transport on $\mathcal{G}$ should in particular be a 1-isomorphism

$$
\mathrm{pt}_{1}^{\mathcal{G}}: \mathrm{ev}_{0}^{*} \mathcal{G} \longrightarrow \mathrm{ev}_{1}^{*} \mathcal{G}
$$

in $\operatorname{BGrb}(P M)$ with a 2-isomorphism

$$
\mathrm{c}^{*} \mathrm{pt} \mathrm{G}_{1}^{\mathcal{G}} \cong 1_{\mathcal{G}},
$$

where c: $M \longrightarrow P M$ is the embedding of $M$ into $P M$ as constant paths. Note that the parallel transport is, in general, an isomorphism of gerbes without connections. The same is true for bundles: the parallel transport on a vector bundle with connection respects the connection if and only if the connection is flat.

To proceed further, we need some definitions. Let $i, n \in \mathbb{N}$ with $1 \leq i \leq n$. For each $s=\left(s_{1}, \ldots, s_{n-1}\right) \in[0,1]^{n-1}$, define a smooth map

$$
\begin{aligned}
\iota_{i ; s}^{n}:[0,1] & \longrightarrow[0,1]^{n} \\
t & \longmapsto\left(s_{1}, \ldots, s_{i-1}, t, s_{i}, \ldots, s_{n-1}\right) .
\end{aligned}
$$

Consider the diffeological spaces $P^{n} M$ which are defined by the sets of all maps

$$
\Sigma:[0,1]^{n} \longrightarrow M
$$

satisfying the following property: for all $i=1, \ldots, n$, there exists $\epsilon_{i}>0$ such the map $\Sigma \circ \iota_{i ; s}^{n}:[0,1] \longrightarrow M$ is locally constant on $\left[0, \epsilon_{i}\right) \sqcup\left(1-\epsilon_{i}, 1\right]$. Note that in a plot of $P^{n} M$, the $\epsilon_{i}$ do not have to be constant over the domain of the plot. The space $P^{n} M$ describes $n$-cubes in $M$ with sitting instants in all directions perpendicular to the faces of $[0,1]^{n}$; that is, $P^{n} M$ describes iterated smooth homotopies of paths with sitting instants in $M$.

We also consider the subspaces $P_{*}^{n} M$ of the diffeological spaces $P^{n} M$ consisting of maps $\Sigma \in P^{n} M$ satisfying the following property: for all $s \in[0,1]^{n-1}$, and for each $j=1, \ldots, n-1$ such that $s_{j} \in\{0,1\}$, the map $\Sigma \circ \iota_{i ; s}^{n}$ is constant for all $i>j$. The space $P_{*}^{n} M$ describes iterated smooth homotopies with fixed endpoints in $M$. For example, $P_{*} M=P M$ is the space of paths with sitting instants, $P_{*}^{2} M$ consists of maps $\Sigma \in P^{2} M$ such that

$$
\Sigma(0, t)=\Sigma(0,0) \quad \text { and } \quad \Sigma(1, t)=\Sigma(1,0)
$$

for all $t \in[0,1]$ and so is the space of homotopies of paths with fixed endpoints in $M$, and an element in $P_{*}^{3} M$ is a family of fixed-ends homotopies between two fixed paths in $M$. We say that an element $\Sigma$ in $P_{*}^{n} M$ or in $P^{n} M$ is thin if its differential $\Sigma_{*}$ has non-maximal $\operatorname{ranks} \operatorname{rk}\left(\Sigma_{* \mid s}\right)<n$ for all $s \in[0,1]^{n}$.

Let $s=\left(s_{1}, \ldots, s_{k}\right) \in[0,1]^{k}$ and $n=k+l$. For $0 \leq i_{1}<\cdots<i_{l} \leq n$, we define a map

$$
\iota_{i_{1}, \ldots, i_{l} ; s}^{n}:[0,1]^{l} \longrightarrow[0,1]^{n}
$$

which inserts the coordinates of $t=\left(t_{1}, \ldots, t_{l}\right) \in[0,1]^{l}$ into the $k$-tuple $s$ such that

$$
\left(\iota_{i_{1}, \ldots, i_{l} ; s}^{n}(t)\right)_{j}=t_{j}
$$


for every $j \in\left\{i_{1}, \ldots, i_{l}\right\}$. The maps $\iota_{i_{1}, \ldots, i_{l} ; s}^{n}:[0,1]^{l} \longrightarrow[0,1]^{n}$ induce maps

$$
\iota_{i_{1}, \ldots, i_{l} ; s}^{n *}: P^{n} M \longrightarrow P^{l} M
$$

which map $P_{*}^{n} M$ to $P_{*}^{l} M$.

For the parallel transport of a bundle gerbe, there should also be a 2-isomorphism

$$
\mathrm{pt}_{2}^{\mathcal{G}}:\left(\iota_{1 ; 0}^{2 *}\right)^{*} \mathrm{pt} \mathrm{G}_{1}^{\mathcal{G}} \longrightarrow\left(\iota_{1 ; 1}^{2 *}\right)^{*} \mathrm{pt} \mathrm{G}_{1}^{\mathcal{G}}
$$

in $\operatorname{BGrb}\left(P_{*}^{2} M\right)$. In other words, any map $\Sigma \in P_{*}^{2} M$ is in particular a smooth map $[0,1]^{2} \longrightarrow M$ from the square to $M$. This map is constant on the vertical edges of the square. Pulling back the isomorphism $p t_{1}^{\mathcal{G}}$ to the horizontal edges of the square gives two 1-morphisms $\mathcal{G}_{\Sigma(0,0)} \longrightarrow \mathcal{G}_{\Sigma(1,0)}$, and the 2-morphism pt $\mathrm{G}_{2}^{\mathcal{G}}$ relates these. The data $\left(\mathrm{pt} \mathrm{G}_{1}^{\mathcal{G}}, \mathrm{pt} \mathrm{G}_{2}^{\mathcal{G}}\right.$ ) are required to satisfy the following two properties, which are motivated by [BW19, BW18, Wal16]:

(1) For any two thin maps $\Sigma, \Sigma^{\prime} \in P_{*}^{2} M$ with $\Sigma \circ \iota_{1 ; s}^{2}=\Sigma^{\prime} \circ \iota_{1 ; s}^{2}$ for $s=0$, 1, there is an equality

$$
\mathrm{pt}_{2 \mid \Sigma}^{\mathcal{G}}=\mathrm{pt}_{2 \mid \Sigma^{\prime}}^{\mathcal{G}}
$$

That is, the 2-morphism $p t_{2}^{\mathcal{G}}$ evaluated on any pair of fixed-ends thin homotopies between any two given paths in $M$ gives the same result.

(2) We further demand that for any thin map $h \in P_{*}^{3} M$, there is an equality

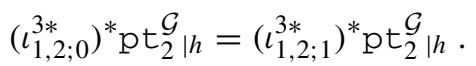

As we will be using $P_{*}^{n} M$ mostly for $n=0,1,2$, we adopt the convention to write $\gamma_{2} \star \gamma_{1}$ for the concatenation of smooth paths in $M$, and if $\Sigma, \Sigma^{\prime} \in P_{*}^{2} M$ are homotopies $\Sigma: \gamma \longrightarrow \gamma^{\prime}$ and $\Sigma^{\prime}: \gamma^{\prime} \longrightarrow \gamma^{\prime \prime}$, we write $\Sigma^{\prime} \star_{2} \Sigma: \gamma \longrightarrow \gamma^{\prime \prime}$ for their vertical concatenation. If $\Xi: \alpha \longrightarrow \alpha^{\prime}$ is a further homotopy in $P_{*}^{2} M$ such that the starting point of $\alpha$ is the endpoint of $\gamma$, then we write $\Xi \star \Sigma: \alpha \star \gamma \longrightarrow \alpha^{\prime} \star \gamma^{\prime}$ for the horizontal concatenation of the homotopies. We will also often use the term 'composition' instead of 'concatenation'.

Definition 4.3. Let $M$ be a smooth manifold. A parallel transport on a bundle gerbe $\mathcal{G} \in \operatorname{BGrb}(M)$ is a quadruple $\mathrm{pt}^{\mathcal{G}}=\left(\mathrm{pt}_{1}^{\mathcal{G}}, \mathrm{pt}{ }_{2}^{\mathcal{G}}, \mathrm{pt}_{\star}^{\mathcal{G}}, \varepsilon^{\mathcal{G}}\right)$ of

(1) a 1-isomorphism

$$
\mathrm{pt}_{1}^{\mathcal{G}}: \mathrm{ev}_{0}^{*} \mathcal{G} \longrightarrow \mathrm{ev}_{1}^{*} \mathcal{G}
$$

of bundle gerbes over $P M$,

(2) a 2-isomorphism

$$
p t_{2}^{\mathcal{G}}:\left(\iota_{1 ; 0}^{2 *}\right)^{*} \mathrm{pt}_{1}^{\mathcal{G}} \longrightarrow\left(\iota_{1 ; 1}^{2 *}\right)^{*} \mathrm{pt}_{1}^{\mathcal{G}}
$$

in $\operatorname{BGrb}\left(P_{*}^{2} M\right)$, 
(3) a 2-isomorphism

$$
\mathrm{pt}_{\star}^{\mathcal{G}}: \mathrm{pr}_{1}^{*} \mathrm{pt}_{1}^{\mathcal{G}} \circ \mathrm{pr}_{2}^{*} \mathrm{pt}_{1}^{\mathcal{G}} \longrightarrow(\cdot \star \cdot)^{*} \mathrm{pt}_{1}^{\mathcal{G}}
$$

over $P M \times_{M} P M$, where $\mathrm{pr}_{1}$ and $\mathrm{pr}_{2}$ are the respective projections of $P M \times_{M} P M$ to the first and second factors, and

(4) a 2-isomorphism

$$
\varepsilon^{\mathcal{G}}: \mathrm{c}^{*} \mathrm{pt}_{1}^{\mathcal{G}} \longrightarrow 1_{\mathcal{G}}
$$

over $M$, where c: $M \longrightarrow P M$ is the inclusion of $M$ as the space of constant paths.

These data are required to satisfy properties (4.1) and (4.2). Due to property (4.1), there is a canonical 2-isomorphism

$$
\mathrm{pt}_{1 \mid\left(\gamma_{3} \star \gamma_{2}\right) \star \gamma_{1}}^{\mathcal{G}} \cong \mathrm{pt}_{1 \mid \gamma_{3} \star\left(\gamma_{2} \star \gamma_{1}\right)}^{\mathcal{G}}
$$

for every $\left(\gamma_{1}, \gamma_{2}, \gamma_{3}\right) \in P M \times_{M} P M \times_{M} P M$, and we demand that $t_{\star}^{\mathcal{G}}$ is coherently associative with respect to this isomorphism. The morphism pt ${ }_{\star}^{\mathcal{G}}$ also needs to be compatible with the unitors in $\operatorname{BGrb}(P M)$ and sit in a commutative diagram

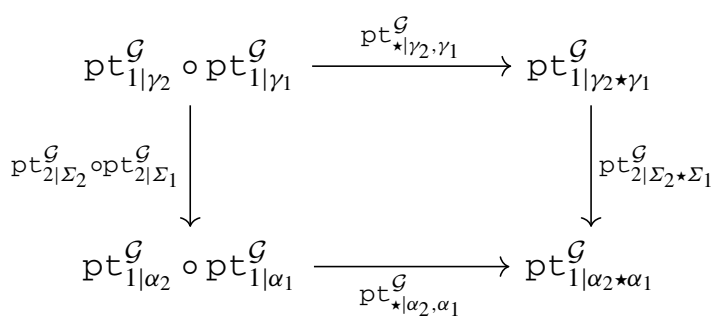

for all $x, y, z \in M$, all paths $\gamma_{1}, \alpha_{1}$ from $x$ to $y$, all paths $\gamma_{2}, \alpha_{2}$ from $y$ to $z$ in $M$, and for all fixed-ends homotopies $\Sigma_{i}: \gamma_{i} \longrightarrow \alpha_{i}$. Furthermore, $\mathrm{pt}_{2}^{\mathcal{G}}$ has to respect vertical composition and satisfy the interchange law

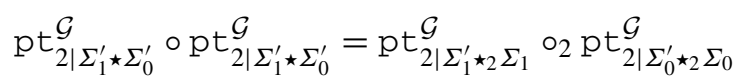

for all points $x_{0}, x_{1}, x_{2} \in M$, all paths $\alpha_{i}, \beta_{i}, \gamma_{i}$ from $x_{i}$ to $x_{i+1}$, and for all fixed-ends homotopies $\Sigma_{i}: \alpha_{i} \longrightarrow \beta_{i}$ and $\Sigma_{i}^{\prime}: \beta_{i} \longrightarrow \gamma_{i}$ with $i=0,1$.

Remark 4.6. The associativity condition in detail reads as follows: for every concatenable triple $\left(\gamma_{1}, \gamma_{2}, \gamma_{3}\right)$ of paths in $M$ there is a commutative diagram

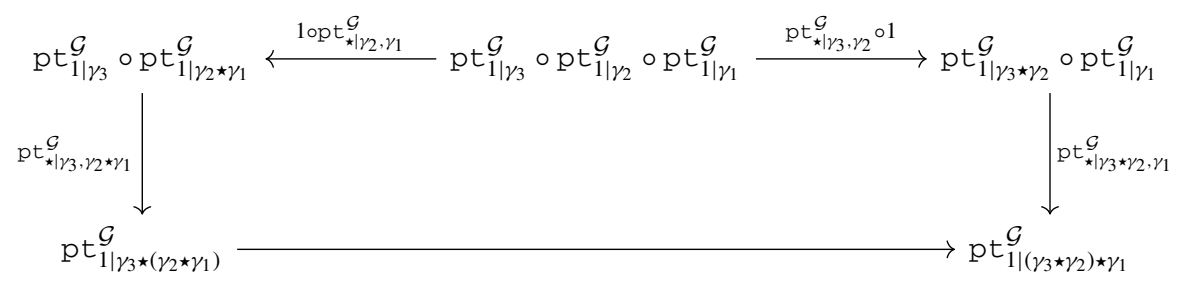

in $\operatorname{BGrb}\left(P M \times{ }_{M} P M\right)$, where the bottom arrow is the canonical 2-isomorphism obtained via (4.1) from any reparameterisation of $[0,1]$ that yields a homotopy $\gamma_{3} \star\left(\gamma_{2} \star \gamma_{1}\right) \sim$ $\left(\gamma_{3} \star \gamma_{2}\right) \star \gamma_{1}$. 
Remark 4.7. By property (4.2), our definition factors through the path 2-groupoid of $M$ as defined by Schreiber and Waldorf [SW11,SW17]. Given a manifold $M$, they construct a 2-groupoid internal to diffeological spaces, whose level sets are essentially $M, P M$ and the quotient $P_{*}^{2} M / \sim$ of $P_{*}^{2} M$ by thin homotopies. Note that in this quotient they also implement condition (4.1).

In contrast to the case of parallel transport on vector bundles, we can define morphisms between parallel transports on a given bundle gerbe.

Definition 4.8. Let $\mathcal{G} \in \operatorname{BGrb}(M)$ be a bundle gerbe on $M$. Let $t^{\mathcal{G}}=\left(\mathrm{pt}_{1}^{\mathcal{G}}, \mathrm{p} t_{2}^{\mathcal{G}}, \mathrm{pt}{ }_{\star}^{\mathcal{G}}\right.$, $\left.{ }_{\varepsilon^{\mathcal{G}}}\right)$ and $\mathrm{pt}^{\prime \mathcal{G}}=\left(\mathrm{pt}_{1}^{\prime} \mathcal{G}, \mathrm{pt}_{2}^{\prime} \mathcal{G}, \mathrm{pt}_{\star}^{\prime} \mathcal{G}, \varepsilon^{\prime \mathcal{G}}\right)$ be two choices of parallel transport on $\mathcal{G}$. A morphism $\mathrm{pt}^{\mathcal{G}} \longrightarrow \mathrm{pt}^{\prime \mathcal{G}}$ of parallel transports on $\mathcal{G}$ is a 2-isomorphism $\psi: \mathrm{pt}_{1}^{\mathcal{G}} \longrightarrow$ $\mathrm{pt}_{1}^{\prime} \mathcal{G}$ in $\operatorname{BGrb}(P M)$ that intertwines the 2-isomorphism $\mathrm{pt}_{2}^{\mathcal{G}}$ with $\mathrm{pt}_{2}^{\prime} \mathcal{G}$, the 2-isomorphism $\mathrm{pt}_{\star}^{\mathcal{G}}$ with $\mathrm{pt}_{\star}^{\prime \mathcal{G}}$, and the 2-isomorphism $\varepsilon^{\mathcal{G}}$ with $\varepsilon^{\prime \mathcal{G}}$. This defines a groupoid $\mathrm{PT}(\mathcal{G})$ of parallel transports on $\mathcal{G}$.

This notion of morphism of parallel transports is not an analogue of a gauge transformation, since it does not necessarily come from an automorphism of the bundle gerbe $\mathcal{G}$.

4.3. Construction of the parallel transport. We now proceed to show that every bundle gerbe with connection on a manifold $M$ has a canonical parallel transport. Let $M$ be a connected manifold, and fix a base point $x \in M$; otherwise, if $M$ is not connected, we treat the connected components of $M$ separately. By results of Waldorf [Wal16], any bundle gerbe $\mathcal{G} \in \mathrm{BGrb}^{\nabla}(M)$ is isomorphic to a bundle gerbe $\mathcal{G}^{\prime} \in \mathrm{BGrb}^{\nabla}(M)$ that is defined over the diffeological path fibration $P_{0} M \longrightarrow M$. Given a choice of base point $x \in M$, Waldorf constructs a bundle gerbe $\mathcal{G}^{\prime}=\mathcal{R} \mathcal{T}(\mathcal{G})$ as the regression of the transgression line bundle of $\mathcal{G}$, together with a natural 1-isomorphism $\mathcal{A}_{\mathcal{G}}: \mathcal{G} \longrightarrow$ $\mathcal{G}^{\prime}$ in the homotopy category of $\operatorname{BGrb}^{\nabla}(M)$; that is, $\mathcal{A}_{\mathcal{G}}$ is determined only up to 2isomorphism. (We remark, however, that the natural 1-isomorphism $\mathcal{A}_{\mathcal{G}}$ from [Wal16] is determined canonically once we fix a preimage of the base point $x \in M$ under the surjective submersion $\pi: Y \longrightarrow M$ underlying the bundle gerbe $\mathcal{G}$.)

Consider the bundle gerbe $\mathcal{G}^{\prime}=\mathcal{R} \mathcal{T}(\mathcal{G}) \in \operatorname{BGrb}^{\nabla}(M)$ with connection on $M$, defined with respect to the path fibration $\pi: P_{0} M \longrightarrow M$. Its line bundle $L$ is the pullback of the transgression line bundle $\mathcal{T} \mathcal{G} \longrightarrow L M$ along the map

$$
\begin{aligned}
\left(P_{0} M\right)^{[2]} & \longrightarrow L M \\
\left(\alpha, \alpha^{\prime}\right) & \longmapsto \overline{\alpha^{\prime}} \star \alpha .
\end{aligned}
$$

By a slight abuse of notation, we also denote this pullback by $\mathcal{T} \mathcal{G} \longrightarrow\left(P_{0} M\right)^{[2]}$.

Construction of $\mathrm{pt}_{1}^{\mathcal{G}^{\prime}}$ We would like to construct a 1-isomorphism

$$
\mathrm{pt}_{1}^{\mathcal{G}^{\prime}}: \mathrm{ev}_{0}^{*} \mathcal{G}^{\prime} \longrightarrow \mathrm{ev}_{1}^{*} \mathcal{G}^{\prime}
$$

in $\operatorname{BGrb}(P M)$. For $t=0,1$, the bundle gerbe $\mathrm{ev}_{t}^{*} \mathcal{G}^{\prime}$ is defined over the subduction $\mathrm{ev}_{t}^{*} P_{0} M \longrightarrow P M$. There are canonical isomorphisms of diffeological spaces

$$
\mathrm{ev}_{0}^{*} P_{0} M \cong P_{0} M \times_{M} P M \quad \text { and } \quad \mathrm{ev}_{1}^{*} P_{0} M \cong P M \times_{M} P_{0} M .
$$


Recall from Sect. 4.1 the space

$$
P_{\partial \Delta^{2}} M:=\operatorname{ev}_{0}^{*} P_{0} M \times{ }_{P M} \mathrm{ev}_{1}^{*} P_{0} M \cong P_{0} M \times_{M} P M \times_{M} P_{0} M .
$$

A point in the total space $P_{\partial \Delta^{2}} M$ is a triple $\left(\alpha_{0}, \gamma, \alpha_{1}\right)$ of a path $\gamma \in P M$ and based paths $\alpha_{t} \in P_{0} M$ such that $\gamma(t)=\alpha_{t}(1)$ for $t=0$, 1 . Any 1 -morphism ev ${ }_{0}^{*} \mathcal{G}^{\prime} \longrightarrow \mathrm{ev}_{1}^{*} \mathcal{G}^{\prime}$ is defined over (possibly a refinement of) the subduction $\xi: P_{\partial \Delta^{2}} M \longrightarrow P M$.

There is a smooth map, i.e. a morphism of diffeological spaces

$$
\begin{aligned}
& \text { s: } P_{\partial \Delta^{2}} M \longrightarrow L M \\
& \left(\alpha_{0}, \gamma, \alpha_{1}\right) \longmapsto \overline{\alpha_{1}} \star\left(\gamma \star \alpha_{0}\right) .
\end{aligned}
$$

There is also the smooth map

$$
\begin{aligned}
& \tilde{\mathrm{s}}: P_{\partial \Delta^{2}} M \longrightarrow L M \\
& \left(\alpha_{0}, \gamma, \alpha_{1}\right) \longmapsto\left(\overline{\alpha_{1}} \star \gamma\right) \star \alpha_{0} .
\end{aligned}
$$

The maps $\mathbf{S}$ and $\tilde{\mathbf{s}}$ are smoothly homotopic via precomposition by a homotopy $h$ of piecewise smooth homeomorphisms $[0,1] \longrightarrow[0,1]$; these fail to be smooth exactly at those points of the interval where the concatenations happen, but at these points all three paths have sitting instants, so that at each time the homotopy maps to $L M$, as desired. For each triple of paths $\left(\alpha_{0}, \gamma, \alpha_{1}\right)$, this results in a thin homotopy in $L M$ from $\overline{\alpha_{1}} \star\left(\gamma \star \alpha_{0}\right)$ to $\left(\overline{\alpha_{1}} \star \gamma\right) \star \alpha_{0}$. By the superficiality of the parallel transport pt ${ }^{\mathcal{T} \mathcal{G}}$ on the transgression line bundle [Wal16, Definition 2.2.1] (see also the end of Sect. 2.2), we thus obtain a canonical isomorphism

$$
r: \mathrm{s}^{*} \mathcal{T} \mathcal{G} \longrightarrow \tilde{\mathrm{s}}^{*} \mathcal{T} \mathcal{G}
$$

in $\operatorname{HLBdl}^{\nabla}\left(P_{\partial \Delta^{2}} M\right)$. The fact that this isomorphism preserves connections is a direct consequence of [Wal16, Lemma 2.3.3]. Since pt ${ }^{\mathcal{T} \mathcal{G}}$ is thin-invariant, it follows that the morphism $r$ is defined independently of the choice of homotopy $h$.

We define a morphism $\mathrm{pt}_{1}^{\mathcal{G}^{\prime}}: \mathrm{ev}_{0}^{*} \mathcal{G}^{\prime} \longrightarrow \mathrm{ev}_{1}^{*} \mathcal{G}^{\prime}$ as follows: its underlying line bundle is the line bundle $\mathrm{s}^{*} \mathcal{T} \mathcal{G} \longrightarrow P_{\partial \Delta^{2}} M$. To turn this into a morphism of bundle gerbes, we need to provide an isomorphism of line bundles

$$
\beta: \operatorname{pr}_{0}^{*} \mathcal{T} \mathcal{G} \otimes \xi_{1}^{*} s^{*} \mathcal{T} \mathcal{G} \longrightarrow \xi_{0}^{*} s^{*} \mathcal{T} \mathcal{G} \otimes \operatorname{pr}_{1}^{*} \mathcal{T} \mathcal{G}
$$

over $\left(P_{\partial \Delta^{2}} M\right)^{[2]}$. Let us unravel this: the fibre product $\left(P_{\partial \Delta^{2}} M\right)^{[2]}=P_{\partial \Delta^{2}} M \times{ }_{P M}$ $P_{\partial \Delta^{2}} M$ consists of pairs $\left(\left(\alpha_{0}, \gamma, \alpha_{1}\right),\left(\alpha_{0}^{\prime}, \gamma, \alpha_{1}^{\prime}\right)\right)$ where $\left(\alpha_{0}, \gamma, \alpha_{1}\right)$ and $\left(\alpha_{0}^{\prime}, \gamma, \alpha_{1}^{\prime}\right)$ are elements of $P_{\partial \Delta^{2}} M$. For $t=0,1$, there are the projection maps

$$
\begin{aligned}
\operatorname{pr}_{t}:\left(P_{\partial \Delta^{2}} M\right)^{[2]} & \longrightarrow\left(P_{0} M\right)^{[2]} \\
\left(\left(\alpha_{0}, \gamma, \alpha_{1}\right),\left(\alpha_{0}^{\prime}, \gamma, \alpha_{1}^{\prime}\right)\right) & \longmapsto\left(\alpha_{t}, \alpha_{t}^{\prime}\right) .
\end{aligned}
$$

Thus

$$
\begin{aligned}
& \left(\operatorname{pr}_{0}^{*} \mathcal{T} \mathcal{G} \otimes \xi_{1}^{*} s^{*} \mathcal{T} \mathcal{G}\right)_{\left(\left(\alpha_{0}, \gamma, \alpha_{1}\right),\left(\alpha_{0}^{\prime}, \gamma, \alpha_{1}^{\prime}\right)\right)}=\mathcal{T} \mathcal{G}_{\overline{\alpha_{0}^{\prime}} \star \alpha_{0}} \otimes \mathcal{T} \mathcal{G}_{\overline{\alpha_{1}^{\prime}} \star\left(\gamma \star \alpha_{0}^{\prime}\right)}, \\
& \left(\xi_{0}^{*} s^{*} \mathcal{T} \mathcal{G} \otimes \operatorname{pr}_{1}^{*} \mathcal{T} \mathcal{G}\right)_{\left(\left(\alpha_{0}, \gamma, \alpha_{1}\right),\left(\alpha_{0}^{\prime}, \gamma, \alpha_{1}^{\prime}\right)\right)}=\mathcal{T} \mathcal{G}_{\overline{\alpha_{1}} \star\left(\gamma \star \alpha_{0}\right)} \otimes \mathcal{T} \mathcal{G}_{\overline{\alpha_{1}^{\prime}} \star \alpha_{1}}
\end{aligned}
$$

Let $\lambda: \pi_{0,1}^{*} \mathcal{T} \mathcal{G} \otimes \pi_{1,2}^{*} \mathcal{T} \mathcal{G} \longrightarrow \pi_{0,2}^{*} \mathcal{T} \mathcal{G}$ denote the fusion product of the transgression line bundle $\mathcal{T} \mathcal{G}$ over $\left(P_{0} M\right)^{[3]}$ (see [Wal16, Section 4.2]), which provides the bundle gerbe 
multiplication on $\mathcal{G}^{\prime}$. At a point $\left(\alpha_{0}, \alpha_{1}, \alpha_{2}\right) \in\left(P_{0} M\right)^{[3]}$ the fusion product consists of unitary isomorphisms

$$
\lambda_{\alpha_{0}, \alpha_{1}, \alpha_{2}}: \mathcal{T} \mathcal{G}_{\overline{\alpha_{1}} \star \alpha_{0}} \otimes \mathcal{T} \mathcal{G}_{\overline{\alpha_{2}} \star \alpha_{1}} \longrightarrow \mathcal{T} \mathcal{G}_{\overline{\alpha_{2}} \star \alpha_{0}} .
$$

The diffeological space $\left(P_{\partial \Delta^{2}} M\right)^{[2]}$ comes with smooth maps

$$
\begin{aligned}
p_{0}:\left(P_{\partial \Delta^{2}} M\right)^{[2]} & \longrightarrow\left(P_{0} M\right)^{[3]} \\
\left(\left(\alpha_{0}, \gamma, \alpha_{1}\right),\left(\alpha_{0}^{\prime}, \gamma, \alpha_{1}^{\prime}\right)\right) & \longmapsto\left(\alpha_{0}, \alpha_{0}^{\prime}, \bar{\gamma} \star \alpha_{1}^{\prime}\right)
\end{aligned}
$$

and

$$
\begin{aligned}
p_{1}:\left(P_{\partial \Delta^{2} M}\right)^{[2]} & \longrightarrow\left(P_{0} M\right)^{[3]} \\
\left(\left(\alpha_{0}, \gamma, \alpha_{1}\right),\left(\alpha_{0}^{\prime}, \gamma, \alpha_{1}^{\prime}\right)\right) & \longmapsto\left(\gamma \star \alpha_{0}, \alpha_{1}^{\prime}, \alpha_{1}\right) .
\end{aligned}
$$

We set

$$
\beta:=p_{1}^{*} \lambda^{-1} \circ r^{-1} \circ p_{0}^{*} \lambda \circ(1 \otimes r) .
$$

Explicitly, at a point $\left(\left(\alpha_{0}, \gamma, \alpha_{1}\right),\left(\alpha_{0}^{\prime}, \gamma, \alpha_{1}^{\prime}\right)\right) \in\left(P_{\partial \Delta^{2}} M\right)^{[2]}$, this is the isomorphism defined by the diagram

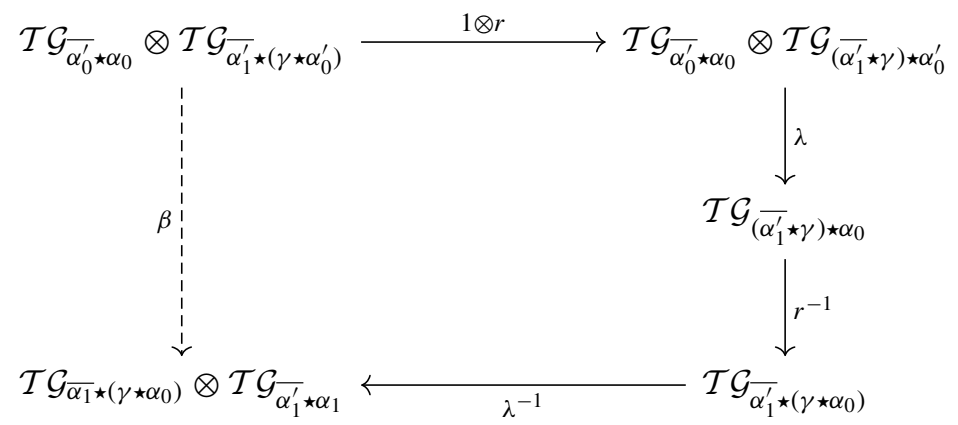

This morphism is compatible with the bundle gerbe multiplication on $\mathcal{G}^{\prime}$ : consider an arbitrary point

$$
\left(\left(\alpha_{0}, \gamma, \alpha_{1}\right),\left(\alpha_{0}^{\prime}, \gamma, \alpha_{1}^{\prime}\right),\left(\alpha_{0}^{\prime \prime}, \gamma, \alpha_{1}^{\prime \prime}\right)\right) \in\left(P_{\partial \Delta^{2}} M\right)^{[3]} .
$$

Then there is a commutative diagram

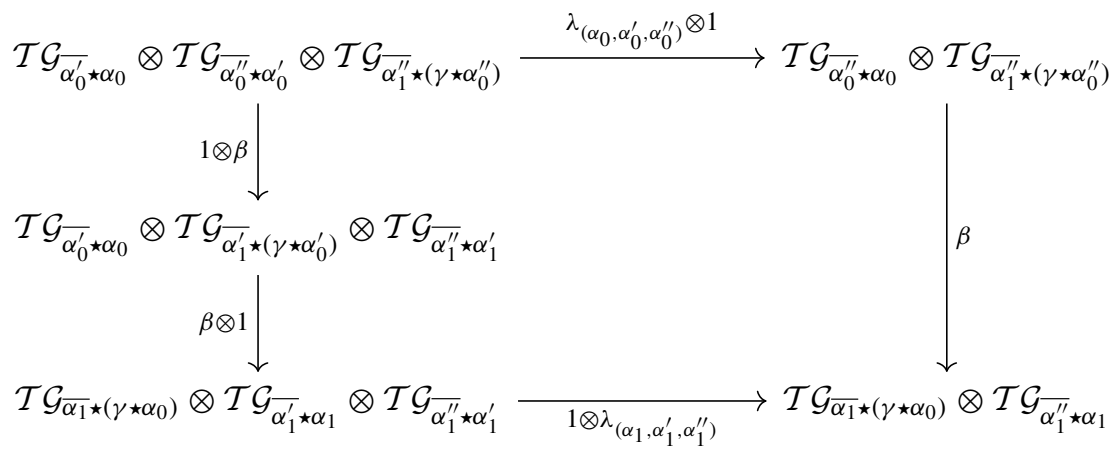

The commutativity follows from the associativity of the fusion product $\lambda$ and the fact that it respects the connection on $\mathcal{T} \mathcal{G}$ [Wal16] so that, in particular, $\lambda$ is compatible with the morphism $r$. 
Construction of $\mathrm{pt}_{2}^{\mathcal{G}^{\prime}}$ Next we construct the 2-isomorphism

$$
\mathrm{pt}_{2}^{\mathcal{G}^{\prime}}:\left(\iota_{1 ; 0}^{2 *}\right)^{*} \mathrm{pt} \mathrm{\mathcal {G }}_{1}^{\prime} \longrightarrow\left(\iota_{1,1}^{2 *}\right)^{*} \mathrm{pt} \mathrm{\mathcal {G }}_{1}^{\prime}
$$

in $\operatorname{BGrb}\left(P_{*}^{2} M\right)$ that is part of the parallel transport data for $\mathcal{G}^{\prime}$. For this, we recall that the fibre of the hermitean line bundle $\mathcal{T G}$ at a loop $\gamma$ is constructed from pairs $([\mathcal{S}], z)$ of a 2-isomorphism class $[\mathcal{S}]$ of trivialisations $\mathcal{S}: \gamma^{*} \mathcal{G} \longrightarrow \mathcal{I}_{0}$ in $\mathrm{BGrb}^{\nabla}\left(\mathbb{S}^{1}\right)$ and a complex number $z \in \mathbb{C}$. The complex line $\mathcal{T} \mathcal{G}_{\gamma}$ is the set of equivalence classes of such pairs under the equivalence relation

$$
([\mathcal{S}], z) \sim\left(\left[\mathcal{S}^{\prime}\right], \operatorname{hol}\left(\mathbb{S}^{1}, \mathrm{R}\left(\mathcal{S}^{\prime} \circ \mathcal{S}^{-1}\right)\right) z\right),
$$

where for a manifold $M$, the functor $\mathrm{R}: \operatorname{BGrb}^{\nabla}(M)\left(\mathcal{I}_{B}, \mathcal{I}_{B^{\prime}}\right) \longrightarrow \operatorname{HLBdl}^{\nabla}(M)$ for $B, B^{\prime} \in \Omega^{2}(M)$ is essentially descent for line bundles; for details, see [Bun17, BSS 18 , Wal07b] (see also Sect. 2.2).

Let $M^{\mathbb{D}^{2}}$ be the diffeological space of smooth maps from the unit disk $\mathbb{D}^{2}$ to $M$. Let

$$
\begin{aligned}
\partial: M^{\mathbb{D}^{2}} & \longrightarrow L M \\
f & \longmapsto f_{\mid \mathbb{S} 1}
\end{aligned}
$$

denote the smooth map induced by restriction to the boundary of the unit disk. The hermitean line bundle $\partial^{*} \mathcal{T} \mathcal{G}$ on $M^{\mathbb{D}^{2}}$ has a canonical trivialisation which is defined as follows: for a smooth map $f: \mathbb{D}^{2} \longrightarrow M$, choose a trivialisation $\mathcal{S}: f^{*} \mathcal{G} \longrightarrow \mathcal{I}_{B}$ for some $B \in \Omega^{2}\left(\mathbb{D}^{2}\right)$. Define a unitary isomorphism of hermitean complex lines

$$
\begin{aligned}
\sigma_{f}: \mathbb{C} & \longrightarrow\left(\partial^{*} \mathcal{T} \mathcal{G}\right)_{f} \\
z & \longmapsto \sigma_{f}(z):=\left[\left[\mathcal{S}_{\mid \mathbb{S} 1}\right], \exp \left(-\mathrm{i} \int_{\mathbb{D}^{2}} B\right) z\right] .
\end{aligned}
$$

This isomorphism is defined independently of the choice of $\mathcal{S}$ : let $\mathcal{S}^{\prime}: f^{*} \mathcal{G} \longrightarrow \mathcal{I}_{B^{\prime}}$ be another trivialisation. Then the line bundle $\mathrm{R}\left(\mathcal{S}^{\prime} \circ \mathcal{S}^{-1}\right)$ has curvature $B^{\prime}-B$, which implies that

$$
\begin{aligned}
\sigma_{f}(z) & :=\left[\left[\mathcal{S}_{\mid \mathbb{S}^{1}}\right], \exp \left(-\mathrm{i} \int_{\mathbb{D}^{2}} B\right) z\right] \\
& =\left[\left[\mathcal{S}_{\mid \mathbb{S}^{1}}\right], \exp \left(-\mathrm{i} \int_{\mathbb{D}^{2}} B^{\prime}\right) \operatorname{hol}\left(\mathbb{S}^{1}, \mathrm{R}\left(\mathcal{S}^{\prime} \circ \mathcal{S}^{-1}\right)\right) z\right] \\
& =\left[\left[\mathcal{S}_{\mid \mathbb{S}^{1}}^{\prime}\right], \exp \left(-\mathrm{i} \int_{\mathbb{D}^{2}} B^{\prime}\right) z\right]
\end{aligned}
$$

This construction works equally well if we replace the 'round' unit disk $\mathbb{D}^{2}$ by the unit square $[0,1]^{2}$, as long as we consider maps $f:[0,1]^{2} \longrightarrow M$ whose restrictions to $\partial[0,1]^{2}$ have sitting instants at the corners. By the construction of the fusion product $\lambda$ on $\mathcal{T} \mathcal{G}$, the section $\sigma$ is compatible with fusion,

$$
\sigma_{f^{\prime} \star 2} f=\lambda\left(\sigma_{f^{\prime}}, \sigma_{f}\right)
$$

for all disks $f, f^{\prime}:[0,1]^{2} \longrightarrow M$ that can be concatenated vertically. (This is merely the statement that the integral over $[0,1]^{2}$ decomposes as the sum $\int_{[0,1]^{2}}=\int_{[0,1] \times\left[0, \frac{1}{2}\right]}$ $\left.+\int_{[0,1] \times\left[\frac{1}{2}, 1\right]} \cdot\right)$ 


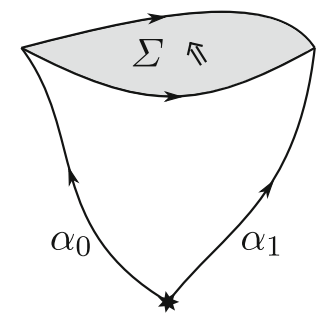

Fig. 2. An element of the space $\widehat{Y}$

Now consider the following setup: let $\Sigma:[0,1]^{2} \longrightarrow M$ be an element in $P_{*}^{2} M$, presenting a fixed-end homotopy from a path $\gamma$ to a path $\gamma^{\prime}$ in $M$. We want to compare the 1-isomorphisms $\left(\iota_{1 ; 0}^{2 *}\right)^{*} \mathrm{pt} \mathrm{\mathcal {G }}_{1}^{\prime}$ and $\left(\iota_{1 ; 1}^{2 *}\right)^{*} \mathrm{pt} \mathcal{G}_{1}^{\prime}$ of bundle gerbes over $P_{*}^{2} M$. The source bundle gerbes of both these morphisms have subductions

$$
Y_{0}:=\left(\iota_{1 ; 0}^{2 *}\right)^{*} \operatorname{ev}_{0}^{*} P_{0} M=\operatorname{ev}_{(0,0)}^{*} P_{0} M=\mathrm{ev}_{(1,0)}^{*} P_{0} M \longrightarrow P_{*}^{2} M,
$$

while the target bundle gerbes live over

$$
Y_{1}:=\left(\iota_{1 ; 0}^{2 *}\right)^{*} \operatorname{ev}_{1}^{*} P_{0} M=\operatorname{ev}_{(0,1)}^{*} P_{0} M=\operatorname{ev}_{(1,1)}^{*} P_{0} M \longrightarrow P_{*}^{2} M .
$$

The fibre product $\widehat{Y}:=Y_{0} \times{ }_{P_{*}^{2} M} Y_{1}$ is the space of triples $\left(\alpha_{0}, \Sigma, \alpha_{1}\right)$ of based paths $\alpha_{0}, \alpha_{1} \in P_{0} M$ and fixed-ends homotopies $\Sigma \in P_{*}^{2} M$ between arbitrary paths in $M$ such that $\alpha_{t}(1)=\Sigma(0, t)$ for $t=0,1$ (see Fig. 2).

The 1-isomorphism $\left(\iota_{1 ; i}^{2 *}\right)^{*} \mathrm{pt}_{1}^{\mathcal{G}^{\prime}}$, for $i=0,1$, is defined over the subduction

$$
Z_{i}:=\left(\iota_{1 ; i}^{2 *}\right)^{*} P_{\partial \Delta^{2}} M \longrightarrow \widehat{Y},
$$

which is actually an isomorphism. Consequently, the 2-isomorphism $p t_{2}^{\mathcal{G}^{\prime}}$ should be defined with respect to the subduction

$$
\widehat{Z}:=Z_{0} \times \widehat{Y} Z_{1} \longrightarrow \widehat{Y},
$$

which again is an isomorphism. Its elements are triples $\left(\alpha_{0}, \Sigma, \alpha_{1}\right)$ as above. Set $\gamma_{t}:=$ $\Sigma \circ \iota_{1 ; t}^{2}$ for $t=0,1$, and let $x=\gamma_{t}(0)$ and $y=\gamma_{s}(1)$ for $t, s=0,1$.

At a point $\left(\alpha_{0}, \Sigma, \alpha_{1}\right)$, the morphism of hermitean line bundles over $\widehat{Z}$ that defines $\mathrm{pt}{ }_{2}^{\mathcal{G}^{\prime}}$ is given by the morphism

$$
\mathrm{pt}_{2 \mid\left(\alpha_{0}, \Sigma, \alpha_{1}\right)}^{\mathcal{G}^{\prime}}: \mathcal{T} \mathcal{G}_{\overline{\alpha_{1}} \star\left(\gamma_{0} \star \alpha_{0}\right)} \longrightarrow \mathcal{T} \mathcal{G}_{\overline{\alpha_{1}} \star\left(\gamma_{1} \star \alpha_{0}\right)}
$$

of complex lines obtained as follows:

(1) Using a smooth family of rotations of $\mathbb{S}^{1}$, apply parallel transport on $\mathcal{T} \mathcal{G}$ to obtain an isomorphism

$$
\left.\psi_{1}: \mathcal{T} \mathcal{G}_{\overline{\alpha_{1}} \star\left(\gamma_{0} \star \alpha_{0}\right)} \longrightarrow \mathcal{T} \mathcal{G}_{\left(\gamma_{0} \star \alpha_{0}\right) \star \overline{\alpha_{1}}} \longrightarrow \mathcal{T} \mathcal{G}_{\gamma_{0} \star\left(\alpha_{0} \star \overline{\alpha_{1}}\right.}\right)
$$

This is achieved by parallel transport along a thin path in $L M$. Hence, since the parallel transport on $\mathcal{T} \mathcal{G}$ is superficial, this isomorphism is independent of the choice of a smooth family of rotations. 
(2) Use the canonical section $\sigma_{\Sigma}(1) \in \mathcal{T} \mathcal{G}_{\partial \Sigma}$ from (4.12) to obtain an isomorphism

$$
\left.\psi_{2}: \mathcal{T} \mathcal{G}_{\gamma_{0} \star\left(\alpha_{0} \star \overline{\alpha_{1}}\right.}\right) \longrightarrow \mathcal{T} \mathcal{G}_{\gamma_{0} \star\left(\alpha_{0} \star \overline{\alpha_{1}}\right)} \otimes \mathcal{T} \mathcal{G}_{\partial \Sigma}
$$

(3) The boundary loop $\partial \Sigma$ is smoothly and thinly homotopic (via reparameterisations) to $\left(\left(\operatorname{id}_{y} \star \gamma_{1}\right) \star \mathrm{id}_{x}\right) \star \overline{\gamma_{0}}$, where $\mathrm{id}_{x}$ is the constant path at the point $x \in M$. This loop is, in turn, thinly homotopic to $\gamma_{1} \star \overline{\gamma_{0}}$. We thus obtain a canonical isomorphism

$$
\psi_{3}: \mathcal{T} \mathcal{G}_{\gamma_{0} \star\left(\alpha_{0} \star \overline{\alpha_{1}}\right)} \otimes \mathcal{T} \mathcal{G}_{\partial \Sigma} \longrightarrow \mathcal{T} \mathcal{G}_{\gamma_{0} \star\left(\alpha_{0} \star \overline{\alpha_{1}}\right)} \otimes \mathcal{T} \mathcal{G}_{\gamma_{1} \star \overline{\gamma_{0}}}
$$

(4) The fusion product on $\mathcal{T} \mathcal{G}$ yields an isomorphism

$$
\psi_{4}: \mathcal{T} \mathcal{G}_{\gamma_{0} \star\left(\alpha_{0} \star \overline{\alpha_{1}}\right)} \otimes \mathcal{T} \mathcal{G}_{\gamma_{1} \star \overline{\gamma_{0}}} \longrightarrow \mathcal{T} \mathcal{G}_{\gamma_{1} \star\left(\alpha_{0} \star \overline{\alpha_{1}}\right)}
$$

(5) Finally, we again use parallel transport along a path in $L M$ that arises from a smooth family of rotations to obtain a canonical isomorphism

$$
\psi_{5}: \mathcal{T} \mathcal{G}_{\gamma_{1} \star\left(\alpha_{0} \star \overline{\alpha_{1}}\right)} \longrightarrow \mathcal{T} \mathcal{G}_{\overline{\alpha_{1}} \star\left(\gamma_{1} \star \alpha_{0}\right)}
$$

We then define

$$
\operatorname{pt}_{2 \mid\left(\alpha_{0}, \Sigma, \alpha_{1}\right)}^{\mathcal{G}^{\prime}}:=\psi_{5} \circ \psi_{4} \circ \psi_{3} \circ \psi_{2} \circ \psi_{1}
$$

This is compatible with vertical composition in $P_{*}^{2} M$ : let $\Sigma, \Sigma^{\prime} \in P_{*}^{2} M$ be two maps $[0,1]^{2} \longrightarrow M$ that can be concatenated vertically. Since the connection on $\mathcal{T} \mathcal{G}$ is superficial and compatible with the fusion product, we can replace the morphism $\psi_{1}$ by

$$
\left.\tilde{\psi}_{1}: \mathcal{T} \mathcal{G}_{\overline{\alpha_{1}} \star\left(l_{1 ; 0}^{2 *} \Sigma \star \alpha_{0}\right)} \longrightarrow \mathcal{T} \mathcal{G}_{l_{1 ; 0}^{2 *} \Sigma \star\left(\alpha_{0} \star \overline{\alpha_{1}}\right.}\right) .
$$

Applying the fusion product with $\partial\left(\Sigma^{\prime} \star_{2} \Sigma\right)$ yields an isomorphism

$$
\varphi: \mathcal{T G}_{l_{1 ; 0}^{2 *} \Sigma \star\left(\alpha_{0} \star \overline{\alpha_{1}}\right)} \longrightarrow \mathcal{T} \mathcal{G}_{l_{1 ; 1}^{2 *} \Sigma^{\prime} \star\left(\alpha_{0} \star \overline{\alpha_{1}}\right)} .
$$

Combining the fact that the fusion product $\lambda$ is associative and compatible with the parallel transport on $\mathcal{T G}$, that the parallel transport on $\mathcal{T} \mathcal{G}$ is superficial (in particular, parallel transport along thin paths is independent of the choice of thin path), and that the section $\sigma$ from (4.12) is compatible with $\lambda$, it follows that $p t_{2}^{\mathcal{G}^{\prime}}$ respects vertical concatenation.

Since all morphisms involved in the construction of $\mathrm{pt}_{2}^{\mathcal{G}^{\prime}}$ are smooth, it follows that $p t_{2}^{\mathcal{G}^{\prime}}$ is in fact a smooth morphism of bundle gerbes as desired.

Construction of $\mathrm{pt}_{\star}^{\mathcal{G}^{\prime}}$ The 2-isomorphism

$$
\mathrm{pt}_{\star}^{\mathcal{G}^{\prime}}: \mathrm{pr}_{1}^{*} \mathrm{pt}_{1}^{\mathcal{G}^{\prime}} \circ \mathrm{pr}_{2}^{*} \mathrm{pt}_{1}^{\mathcal{G}^{\prime}} \longrightarrow(\cdot \star \cdot)^{*} \mathrm{pt}_{1}^{\mathcal{G}^{\prime}}
$$

is directly constructed from the fusion product $\lambda$ on the transgression line bundle $\mathcal{T} \mathcal{G}$. Define $q: P M \times_{M} P M \longrightarrow M$ by $\left(\gamma, \gamma^{\prime}\right) \longmapsto \gamma(0)=\gamma^{\prime}(1)$. The morphism pt $t_{\star}^{\mathcal{G}^{\prime}}$ is defined over the subduction

$$
Q_{1}:=\operatorname{pr}_{1}^{*} P_{\partial \Delta^{2}} M \times{ }_{q^{*}} P_{0} M \operatorname{pr}_{2}^{*} P_{\partial \Delta^{2}} M \longrightarrow P M \times_{M} P M .
$$


Given a point $\left(\left(\alpha_{0}, \gamma, \alpha_{1}\right),\left(\alpha_{1}, \gamma^{\prime}, \alpha_{2}\right)\right) \in Q_{1}$, the morphism $\mathrm{pt}_{\star}^{\mathcal{G}^{\prime}}$ is given by the diagram

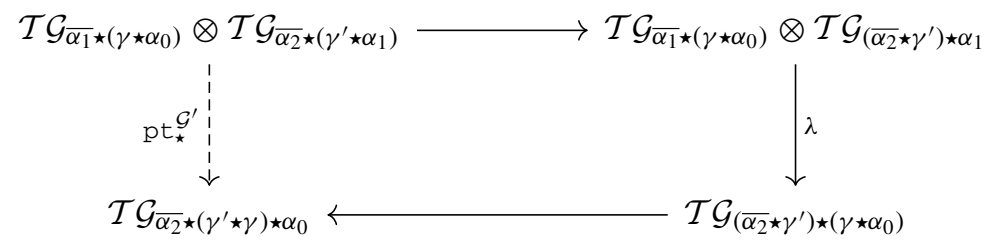

where the horizontal morphisms are induced by smooth families of reparameterisations. The compatibility of this morphism with the morphism $\beta$ from (4.11) follows again from the superficiality of the connection on $\mathcal{T} \mathcal{G}$ and the associativity of the fusion product $\lambda$.

The compatibility of $p t_{\star}^{\mathcal{G}^{\prime}}$ with $p t_{2}^{\mathcal{G}^{\prime}}$ as in (4.4) is seen analogously to how we proved the compatibility of $\mathrm{pt}_{2}^{\mathcal{G}^{\prime}}$ with vertical concatenation of homotopies. The interchange law (4.5) is satisfied by the associativity of $\lambda$, its compatibility with the parallel transport on $\mathcal{T} \mathcal{G}$ and with the section $\sigma$ from (4.12), as well as the superficiality of the connection on $\mathcal{T} \mathcal{G}$.

Construction of $\varepsilon^{\mathcal{G}^{\prime}}$ Finally, the 2-isomorphism<smiles>[CH]=[C+][Te][Te]</smiles>

is obtained directly from the superficial connection on $\mathcal{T} \mathcal{G}$ : it is defined over the space of triples $\left(\alpha, \mathrm{id}_{x}, \alpha\right) \in P_{\partial \Delta^{2}} M$, and all paths of the form $\bar{\alpha} \star \alpha$ are canonically contractible by thin homotopies.

All necessary coherences in Definition 4.3 then follow from the superficiality of the parallel transport on $\mathcal{T} \mathcal{G}$, the associativity of the fusion product $\lambda$ and its compatibility with the section $\sigma$, and the fact that the parallel transport on $\mathcal{T} \mathcal{G}$ is compatible with the fusion product. Thus we have

Theorem 4.13. Let $\mathcal{G} \in \mathrm{BGrb}^{\nabla}(M)$ be a bundle gerbe with connection on $M$, and let $\mathcal{G}^{\prime}:=\mathcal{R} \mathcal{T}(\mathcal{G}) \in \operatorname{BGrb}^{\nabla}(M)$ be the regression of the transgression of $\mathcal{G}$. Then the quadruple $\mathrm{pt}^{\mathcal{G}^{\prime}}=\left(\mathrm{p} t_{1}^{\mathcal{G}^{\prime}}, \mathrm{p} t_{2}^{\mathcal{G}^{\prime}}, \mathrm{pt}{ }_{\star}^{\mathcal{G}^{\prime}}, \varepsilon^{\mathcal{G}^{\prime}}\right)$ defines a parallel transport on the bundle gerbe $\mathcal{G}^{\prime}$.

Transfer to arbitrary bundle gerbes In [Wal16], Waldorf shows that the functors $\mathcal{T}$ and $\mathcal{R}$ come with a canonical natural isomorphism

$$
\mathcal{A}: 1 \longrightarrow \mathcal{R} \circ \mathcal{T}
$$

as endofunctors of the homotopy 1-category $\mathrm{hBGrb}^{\nabla}(M)$. Given a bundle gerbe $\mathcal{G} \in$ $\operatorname{BGrb}^{\nabla}(M)$, we thus get a 2 -isomorphism class of 1-isomorphisms $\mathcal{G} \longrightarrow \mathcal{G}^{\prime}=\mathcal{R} \mathcal{T}(\mathcal{G})$. Let $\mathcal{A}_{\mathcal{G}}: \mathcal{G} \longrightarrow \mathcal{G}^{\prime}$ be a representative for this class.

Let $\mathcal{B}: \mathcal{G} \longrightarrow \mathcal{G}^{\prime}$ be a 1-isomorphism with adjoint inverse $\mathcal{B}^{-1}$, i.e. a weak inverse $\mathcal{B}^{-1}$ together with 2-isomorphisms $\epsilon_{\mathcal{B}}: 1_{\mathcal{G}} \longrightarrow \mathcal{B}^{-1} \circ \mathcal{B}$ and $\delta_{\mathcal{B}}: \mathcal{B} \circ \mathcal{B}^{-1} \longrightarrow 1_{\mathcal{G}^{\prime}}$ 
that satisfy the triangle identities. We can use it to define a 1 -isomorphism $p t_{1}^{\mathcal{G}, \mathcal{B}}$ as the composition

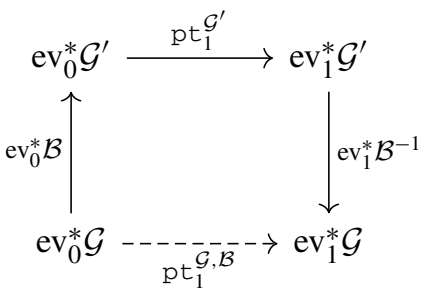

We define 2-isomorphisms

$$
\begin{aligned}
& \mathrm{pt}_{2}^{\mathcal{G}, \mathcal{B}}:=1_{\mathcal{B}^{-1}} \circ \mathrm{pt}_{2}^{\mathcal{G}^{\prime}} \circ 1_{\mathcal{B}}, \\
& p t_{\star}^{\mathcal{G}, \mathcal{B}}:=1_{\mathcal{B}^{-1}} \circ\left(\mathrm{pt}_{\star}^{\mathcal{G}^{\prime}} \circ_{2}\left(1_{p t_{1}^{\mathcal{G}^{\prime}}} \circ \delta_{\mathcal{B}} \circ 1_{p t_{1}^{\mathcal{G}^{\prime}}}\right)\right) \circ 1_{\mathcal{B}}, \\
& \varepsilon^{\mathcal{G}, \mathcal{B}}:=\epsilon_{\mathcal{B}} \circ_{2}\left(1_{\mathcal{B}^{-1}} \circ \varepsilon^{\mathcal{G}^{\prime}} \circ 1_{\mathcal{B}}\right),
\end{aligned}
$$

where we have omitted pullbacks. From these definitions we readily see

Proposition 4.14. The quadruple $\mathrm{p} t^{\mathcal{G}, \mathcal{B}}=\left(\mathrm{p} t_{1}^{\mathcal{G}, \mathcal{B}}, \mathrm{p} t_{2}^{\mathcal{G}, \mathcal{B}}, \mathrm{p} t_{\star}^{\mathcal{G}, \mathcal{B}},{ }_{\varepsilon}^{\mathcal{G}, \mathcal{B}}\right)$ defines an object in $\mathrm{PT}(\mathcal{G})$.

For $\psi: \mathcal{B} \longrightarrow \mathcal{B}^{\prime}$ a 2-isomorphism of 1-isomorphisms $\mathcal{B}, \mathcal{B}^{\prime}: \mathcal{G}^{\prime} \longrightarrow \mathcal{G}$, we obtain a 2-isomorphism

$$
\psi^{(-1)} \circ 1_{p t_{1}^{\mathcal{G}^{\prime}}} \circ \psi: p t_{1}^{\mathcal{G}, \mathcal{B}} \longrightarrow p t_{1}^{\mathcal{G}, \mathcal{B}^{\prime}}
$$

Here $\psi^{(-1)}$ denotes the 2-isomorphism obtained from $\psi$ by taking the inverse with respect to horizontal composition. Again it follows from the definitions that this defines an isomorphism

$$
\widehat{\psi}: \mathrm{p} t^{\mathcal{G}, \mathcal{B}} \longrightarrow \mathrm{p} t^{\mathcal{G}, \mathcal{B}^{\prime}}
$$

in the category $\mathrm{PT}(\mathcal{G})$. If $\psi^{\prime}: \mathcal{B} \longrightarrow \mathcal{B}^{\prime}$ is another (parallel unitary) 2-isomorphism, then $\psi$ and $\psi^{\prime}$ differ by multiplication with a locally constant U(1)-valued function $f_{\psi, \psi^{\prime}}$ on $M$. Since horizontal inverses of 2-isomorphisms have dual underlying line bundles [Wal07b], the morphisms $\psi^{(-1)}$ and $\psi^{\prime(-1)}$ differ by the locally constant U(1)valued function $f_{\psi^{(-1)}, \psi^{\prime(-1)}}=\left(f_{\psi, \psi^{\prime}}\right)^{-1}$. Consequently, we deduce from (4.15) that

$$
\widehat{\psi^{\prime}}=\widehat{\psi} .
$$

That is, for any pair of parallel unitary 1 -isomorphisms $\mathcal{B}, \mathcal{B}^{\prime}: \mathcal{G} \longrightarrow \mathcal{G}^{\prime}$ for which there exists some parallel unitary 2 -isomorphism $\mathcal{B} \longrightarrow \mathcal{B}^{\prime}$, we obtain a unique isomorphism $\mathrm{pt}^{\mathcal{G}, \mathcal{B}} \longrightarrow \mathrm{pt}^{\mathcal{G}, \mathcal{B}^{\prime}}$

Let $\left[\left[\mathcal{A}_{\mathcal{G}}\right]\right]$ denote the full subgroupoid of $\operatorname{BGrb}^{\nabla}(M)\left(\mathcal{G}, \mathcal{G}^{\prime}\right)$ on those 1-isomorphisms $\mathcal{G} \longrightarrow \mathcal{G}^{\prime}$ that are isomorphic to Waldorf's 1-isomorphism $\mathcal{A}_{\mathcal{G}}$. Our constructions define a functor

$$
\mathrm{pt}^{\mathcal{G},(\cdot)}:\left[\left[\mathcal{A}_{\mathcal{G}}\right]\right] \longrightarrow \mathrm{PT}(\mathcal{G}) \text {. }
$$


This functor factors through a groupoid $\left[\left[\mathcal{A}_{\mathcal{G}}\right]\right]_{*}$ with the same objects as $\left[\left[\mathcal{A}_{\mathcal{G}}\right]\right]$ and a unique isomorphism between any two objects. In particular, every object in $\left[\left[\mathcal{A}_{\mathcal{G}}\right]\right]_{*}$ is final and the canonical functor $\left[\left[\mathcal{A}_{\mathcal{G}}\right]\right] \longrightarrow\left[\left[\mathcal{A}_{\mathcal{G}}\right]\right]_{*}$ is a final functor. It follows that, for any category $\mathcal{C}$, any functor $\mathcal{F}:\left[\left[\mathcal{A}_{\mathcal{G}}\right]\right] \longrightarrow \mathcal{C}$ that factors through $\left[\left[\mathcal{A}_{\mathcal{G}}\right]\right]_{*}$ has a colimit, which is represented by $\mathcal{F}(\mathcal{B})$ for any object $\mathcal{B} \in\left[\left[\mathcal{A}_{\mathcal{G}}\right]\right]$.

Definition 4.16. Let $\mathcal{G} \in \operatorname{BGrb}^{\nabla}(M)$ be a bundle gerbe with connection on $M$. The parallel transport of $\mathcal{G}$ is

$$
\mathrm{pt}^{\mathcal{G}}:=\operatorname{colim}\left(\mathrm{pt}^{\mathcal{G},(\cdot)}:\left[\left[\mathcal{A}_{\mathcal{G}}\right]\right] \longrightarrow \mathrm{PT}(\mathcal{G})\right) .
$$

4.4. The transgression line bundle as a holonomy. Let $\mathcal{G} \in \mathrm{BGrb}^{\nabla}(M)$ be a bundle gerbe with connection on $M$ and write $\mathcal{G}^{\prime}=\mathcal{R} \mathcal{T}(\mathcal{G})$. We will now determine the holonomy of the parallel transport on $\mathcal{G}^{\prime}$. For this, consider the diffeological space $L_{*} M$ of smooth maps $\mathbb{S}^{1} \longrightarrow M$ that have a sitting instant at $1 \in \mathbb{S}^{1}$. In other words, $L_{*} M$ is the pullback

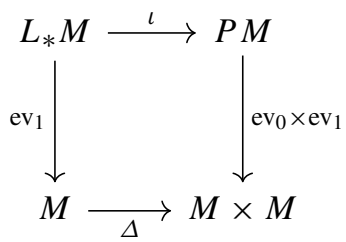

in $\mathcal{D}$ fg, where $\iota$ denotes the inclusion map and $\Delta$ is the diagonal embedding. The pullback $\iota^{*} \mathrm{pt} \mathcal{G}_{1}^{\prime}$ is an automorphism of $\mathrm{ev}_{1}^{*} \mathcal{G}^{\prime}$, which we understand as the holonomy of pt $\mathcal{G}^{\prime}$. It is defined over the subduction $\iota^{*} P_{\partial \Delta^{2}} M \longrightarrow L_{*} M$. Recall from Sect. 2.2 that a 1-automorphism of a bundle gerbe defines a line bundle via descent. Thus the holonomy $\iota^{*} \mathrm{pt}{ }_{1}^{\mathcal{G}^{\prime}}$ gives rise to a descended line bundle $\operatorname{hol}(\mathcal{G}) \in \operatorname{HLBdl}\left(L_{*} M\right)$. Our goal is to understand this descended line bundle more explicitly.

Let $\widehat{\mathrm{ev}_{1}}: \mathrm{ev}_{1}^{*} P_{0} M \longrightarrow P_{0} M$ be the morphism induced by the pullback

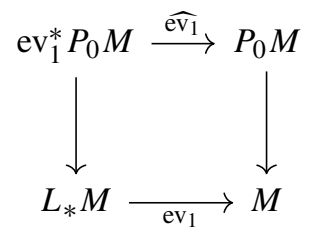

in $\mathcal{D}$ fg. The hermitean line bundle (with connection) underlying the bundle gerbe ev $\mathcal{G}^{\prime}$ is the pullback bundle

$$
L:=\widehat{\mathrm{ev}}_{1}^{[2] *} \mathcal{T} \mathcal{G} \longrightarrow\left(\mathrm{ev}_{1}^{*} P_{0} M\right)^{[2]} \cong \iota^{*} P_{\partial \Delta^{2}} M
$$

We now apply the construction from the diagram (2.18) that produces a line bundle $\mathrm{R}(\mathcal{A})$ from an automorphism $\mathcal{A}$ of a bundle gerbe: the tensor product bundle $L^{\vee} \otimes \mathrm{p} t_{1}^{\mathcal{G}^{\prime}}$ on $\iota^{*} P_{\partial \Delta^{2}} M$ has fibres

$$
\left(L^{\vee} \otimes \mathrm{pt}_{1}^{\mathcal{G}^{\prime}}\right)_{\left(\alpha_{0}, \gamma, \alpha_{1}\right)}=\mathcal{T} \mathcal{G}_{\overline{\alpha_{1}} \star \alpha_{0}}^{\vee} \otimes \mathcal{T} \mathcal{G}_{\overline{\alpha_{1}} \star\left(\gamma \star \alpha_{0}\right)} \cong \mathcal{T} \mathcal{G}_{\overline{\alpha_{0}} \star \alpha_{1}} \otimes \mathcal{T} \mathcal{G}_{\overline{\alpha_{1}} \star\left(\gamma \star \alpha_{0}\right)}
$$


Now the thin-invariant parallel transport and the fusion product on $\mathcal{T G}$ yield an isomorphism

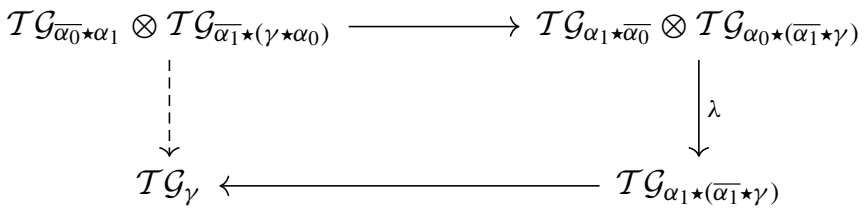

By the associativity of $\lambda$, this is an isomorphism of descent data for line bundles on $L_{*} M$. This shows

Proposition 4.18. The morphism (4.17) yields an isomorphism of hermitean line bundles

$$
\operatorname{hol}(\mathcal{G}):=\mathrm{R}\left(\iota^{*} \mathrm{p} t_{1}^{\mathcal{G}^{\prime}}\right) \stackrel{\cong}{\longrightarrow} \mathcal{T} \mathcal{G}_{\mid L_{*} M}
$$

over $L_{*} M$.

Thus the parallel transport $\mathrm{t}^{\mathcal{G}^{\prime}}$ reproduces the transgression line bundle $\mathcal{T} \mathcal{G}$ as its holonomy.

Remark 4.19. For a generic bundle gerbe $\mathcal{G}$ with parallel transport pt ${ }^{\mathcal{G}}$, the morphism pt $\frac{\mathcal{G}}{2}$ induces a parallel transport on $\operatorname{hol}(\mathcal{G})$. It should be possible to construct from this a fusion line bundle with connection on $L M$ in the sense of [Wal16], which then regresses to a bundle gerbe with connection on $M$. Its underlying bundle gerbe should be canonically isomorphic to $\mathcal{G}$ (up to 2-isomorphism), and that should allow the reconstruction of the connection on $\mathcal{G}$ from its parallel transport in our sense. However, this would go beyond the scope of this paper, and since for our applications in Sects. 5 and 6 having an explicit construction for $p t^{\mathcal{G}^{\prime}}$ is sufficient, we leave this reconstruction of the connection on $\mathcal{G}$ for future work.

\section{2-Group Extensions from Bundle Gerbes}

Let $G$ be a connected Lie group with a smooth group action on a manifold $M$. In Sect. 3 we saw how a principal bundle $P \longrightarrow M$ gives rise to a group extension $\operatorname{Sym}_{G}(P) \longrightarrow G$ which encodes all information about equivariant structures on $P$. We were able to give two equivalent constructions for $\operatorname{Sym}_{G}(P)$, one as a subgroup of $\operatorname{Diff}(P)$, and one as descent data associated to the path fibration $P_{0} G \longrightarrow G$ and a parallel transport on $P$.

In this section we study the analogous situation for a bundle gerbe $\mathcal{G} \in \operatorname{BGrb}(M)$ instead of a principal bundle $P \in \operatorname{Bun}_{H}(M)$. There are two main differences to the situation in Sect. 3: equivariant structures on $\mathcal{G}$ form a groupoid rather than a set, and they do not assemble into a topological or smooth space. We thus cannot expect a universal extension $\operatorname{Sym}_{G}(\mathcal{G}) \longrightarrow G$ as diffeological groups. A good framework to describe this extension is that of group objects in categories fibred in groupoids over Cart, where the fibration encodes the smooth structure. After carefully setting up this framework, we give two constructions of $\operatorname{Sym}_{G}(\mathcal{G})$, in analogy to the two constructions of $\operatorname{Sym}_{G}(P)$ in Sect. 3. We conclude this section by showing that, again, the extension $\operatorname{Sym}_{G}(\mathcal{G}) \longrightarrow G$ encodes all information about equivariant structures on $\mathcal{G}$. 
5.1. Smooth groupoids and symmetries of gerbes. Let $\mathcal{G} \in \mathrm{BGrb}(M)$ be a bundle gerbe on $M$. Let $\Phi: G \times M \longrightarrow M$ be an action of a connected Lie (or diffeological) group $G$ on $M$. Let Cart denote the category of smooth manifolds that are diffeomorphic to $\mathbb{R}^{n}$ for some $n \in \mathbb{N}_{0}$. The morphisms in Cart are the smooth maps between these manifolds.

We can view $M$ and $G$ as presheaves on Cart by setting

$$
M(c)=C^{\infty}(c, M) \quad \text { and } \quad G(c)=C^{\infty}(c, G) .
$$

By adding identity morphisms, we can canonically enhance the presheaf $G$ to a (pre)stack on Cart, i.e. a (pre)sheaf of groupoids, which we still denote by $G$. Given a section $f \in G(c)$, i.e. a smooth map $f: c \longrightarrow G$, we can define a map

$$
\Phi_{f}: c \times M \stackrel{f \times 1_{M}}{\longrightarrow} G \times M \stackrel{\Phi}{\longrightarrow} M .
$$

We can then assign to $f$ the groupoid

$$
\operatorname{Sym}_{G}^{\mathrm{PSh}}(\mathcal{G})(f):=\operatorname{BGrb}(c \times M)\left(\operatorname{pr}_{M}^{*} \mathcal{G}, \Phi_{f}^{*} \mathcal{G}\right),
$$

where $\operatorname{pr}_{M}: c \times M \longrightarrow M$ is the projection. The groupoid $\operatorname{Sym}_{G}^{\mathrm{PSh}}(\mathcal{G})(f)$ is nonempty: since the map $f: c \longrightarrow G$ is homotopic to the constant map at the identity in $G$, it follows that $\operatorname{pr}_{M}^{*} \mathcal{G}$ and $\Phi_{f}^{*} \mathcal{G}$ have the same Dixmier-Douady class as gerbes on $c \times M$, so that there exists an isomorphism $\operatorname{pr}_{M}^{*} \mathcal{G} \longrightarrow \Phi_{f}^{*} \mathcal{G}$.

The assignment $f \longmapsto \operatorname{Sym}_{G}^{\text {PSh }}(\mathcal{G})(f)$ is evidently not a presheaf of groupoids on Cart since it depends not only on the object $c$, but also on a choice of a smooth map $f: c \longrightarrow G$. We can reformulate this in the following way: let $\underline{G}$ denote the category with objects the smooth maps $f: c \longrightarrow G$, where $c \in$ Cart is any Cartesian space. The morphisms

$$
\varphi:(f: c \longrightarrow G) \longrightarrow\left(f^{\prime}: c^{\prime} \longrightarrow G\right)
$$

in $\underline{G}$ are commutative triangles of smooth maps

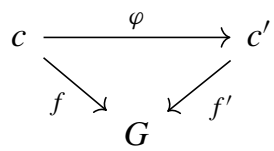

Then $\operatorname{Sym}_{G}^{\text {PSh }}(\mathcal{G})$ is a presheaf of groupoids on $\underline{G}$ : to an object $f: c \longrightarrow G$ in $\underline{G}$ we assign the groupoid $\operatorname{Sym}_{G}^{\mathrm{PSh}}(\mathcal{G})(f)$, while to a morphism $\varphi: f \longrightarrow f^{\prime}$ we assign the pullback functor

$$
\operatorname{Sym}_{G}^{\mathrm{PSh}}(\mathcal{G})(\varphi):=\left(\varphi \times 1_{M}\right)^{*}: \operatorname{Sym}_{G}^{\mathrm{PSh}}(\mathcal{G})\left(f^{\prime}\right) \longrightarrow \operatorname{Sym}_{G}^{\mathrm{PSh}}(\mathcal{G})(f) .
$$

By a slight abuse of notation, we will denote the functor $\operatorname{Sym}_{G}^{\mathrm{PSh}}(\mathcal{G})(\varphi)$ by $\varphi^{*}$. Explicitly, given a 1-isomorphism $A: \operatorname{pr}_{M}^{*} \mathcal{G} \longrightarrow \Phi_{f^{\prime}}^{*} \mathcal{G}$ over $c^{\prime}$, it is defined by the commutative diagram

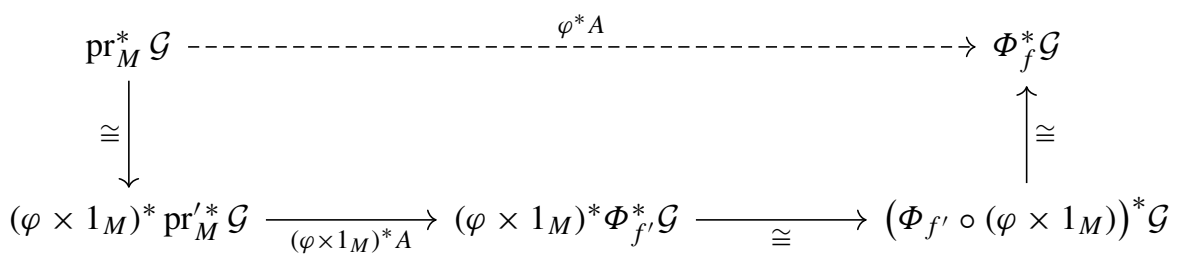


By construction of the 2-category of bundle gerbes, this defines a pseudofunctor

$$
\operatorname{Sym}_{G}^{\mathrm{PSh}}(\mathcal{G}): \underline{G}^{\mathrm{op}} \longrightarrow \operatorname{Grpd},
$$

where Grpd is the 2-category of groupoids, functors, and natural transformations.

Remark 5.1. The assignment $f \longmapsto \operatorname{Sym}_{G}^{\text {PSh }}(\mathcal{G})(f)$ is not a strict presheaf of groupoids on $\underline{G}$, as it is only pseudofunctorial [Moe02]. There are several ways to technically treat such pseudo-presheaves of groupoids:

(1) Encode the coherence morphisms by viewing pseudo-presheaves of groupoids as coherent simplicial presheaves, i.e. as simplicial functors $\mathfrak{C} \circ \mathrm{N}_{\Delta}(\underline{G})^{\text {op }} \longrightarrow \operatorname{Set}_{\Delta}$ in the notation of [Lur09].

(2) Use a strictification procedure to translate pseudo-presheaves of groupoids into presheaves of groupoids [Hol08].

(3) Use the Grothendieck construction, or straightening, to translate pseudo-presheaves of groupoids into categories fibred in groupoids over $\underline{G}$ [Vis05, Lur09].

We will follow the third approach here because the transition between the parameterising categories $\underline{G}$ and Cart becomes particularly easy in that framework.

We will frequently make use of the Grothendieck construction to pass from Grpdvalued pseudo-functors to categories fibred in groupoids; for background on the Grothendieck construction and fibred categories we refer to [Vis05,Lur09]. We will, however, describe the resulting fibred categories explicitly. For example, the canonical projection functor $\mathrm{pr}: \underline{G} \longrightarrow$ Cart is the category fibred in groupoids obtained by applying the Grothendieck construction to the (pseudo)functor $c \longmapsto G(c)$, where $G(c)$ is regarded as a groupoid with only identity arrows.

Definition 5.2. A functor $\pi: \mathcal{D} \longrightarrow \mathcal{C}$ between categories is a Grothendieck fibration in groupoids, or makes $\mathcal{D}$ into a category fibred in groupoids over $\mathcal{C}$, if it satisfies the properties:

(1) For every object $d \in \mathcal{D}$ and for every morphism $f: c \longrightarrow \pi(d)$ in $\mathcal{C}$, there exists a morphism $\widehat{f}: \widehat{c} \longrightarrow d$ in $\mathcal{D}$ with $\pi(\widehat{f})=f$.

(2) For every pair of diagrams
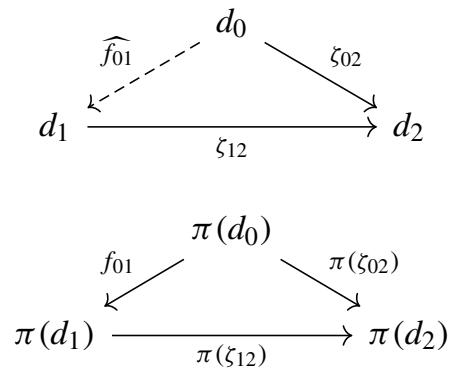

in $\mathcal{D}$ and $\mathcal{C}$, respectively, there exists a unique lift $\widehat{f_{01}}$ of $f_{01}$ that makes the upper triangle commute.

The first requirement resembles a path-lifting condition. The second requirement can be viewed as a relative horn-filling property: given any $\Lambda_{2}^{2}$-horn $\sigma$ in $\mathcal{D}$ and a filling of $\pi(\sigma)$ in $\mathcal{C}$ to a 2-simplex, there exists a unique filling of $\sigma$ to a 2 -simplex in $\mathcal{D}$ 
that lifts the 2-simplex in $\mathcal{C}$. Alternatively, consider an arbitrary functor $\pi: \mathcal{D} \longrightarrow \mathcal{C}$ between categories and a morphism $\zeta_{12}: d_{1} \longrightarrow d_{2}$ in $\mathcal{D}$. If for every pair of solid arrow diagrams as in (5.3) the dashed arrow exists such that the upper triangle commutes and such that $\pi\left(\widehat{f_{01}}\right)=f_{01}$, one says that $\zeta_{12}$ is $\pi$-Cartesian. In particular, if $\pi$ is a Grothendieck fibration in groupoids, then property (2) of Definition 5.2 is equivalent to saying that every morphism in $\mathcal{D}$ is $\pi$-Cartesian. If $\pi: \mathcal{D} \longrightarrow \mathcal{C}$ is a Grothendieck fibration in groupoids and $c \in \mathcal{C}$, we denote by $\mathcal{D}_{\mid c}=\pi^{-1}(c)$ the fibre over $c$, which is the groupoid with objects $d \in \mathcal{D}$ such that $\pi(d)=c$ and morphisms $\widehat{f}: d \longrightarrow d^{\prime}$ such that $\pi(\widehat{f})=1_{c}$.

Definition 5.4. A category fibred in groupoids over Cart is a smooth groupoid. Let $\mathcal{H}$ denote the strict 2-category of smooth groupoids. Its objects are smooth groupoids, its morphisms are (strictly) commutative diagrams of functors

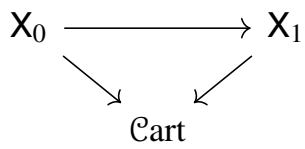

and its 2-morphisms are natural transformations that project to the identity. We denote by $\underline{\mathcal{H}}(\mathrm{X}, \mathrm{Z})$ the groupoid of functors $\mathrm{X} \longrightarrow \mathrm{Z}$ that project to the identity on Cart.

Example 5.5. Let $M$ be a smooth manifold. An important example of a smooth groupoid is given by the Grothendieck fibration $\mathrm{HLBdl}^{M} \longrightarrow$ Cart, whose objects are pairs $(c, L)$ of a Cartesian space $c \in$ Cart and a hermitean line bundle $L \longrightarrow c \times M$, and whose morphisms $(c, L) \longrightarrow\left(c^{\prime}, L^{\prime}\right)$ are pairs $(\varphi, \psi)$ of a smooth map $\varphi: c \longrightarrow c^{\prime}$ and an isomorphism $\psi: L \longrightarrow\left(\varphi \times 1_{M}\right)^{*} L^{\prime}$ of hermitean line bundles on $c \times M$. One can interpret $\mathrm{HLBdl}^{M}$ as describing smooth families of hermitean line bundles on $M$. For $M=*$, we write HLBdl* $=$ : HLBdl.

Definition 5.6. Let $p: \operatorname{Sym}_{G}(\mathcal{G}) \longrightarrow \underline{G}$ denote the category fibred in groupoids obtained by applying the Grothendieck construction to the pseudofunctor $\operatorname{Sym}_{G}^{\mathrm{PSh}}(\mathcal{G}): \underline{G}^{\mathrm{op}} \longrightarrow$ Grpd. Explicitly, the category $\operatorname{Sym}_{G}(\mathcal{G})$ consists of:

- Objects : pairs $(f, A)$, where $f \in \underline{G}$ is a smooth map $f: c \longrightarrow G$ and $A: \operatorname{pr}_{M}^{*} \mathcal{G} \longrightarrow$ $\Phi_{f}^{* \mathcal{G}}$ is a 1 -isomorphism of bundle gerbes over $c \times M$.

- Morphisms : a morphism $\left(f_{0}, A_{0}\right) \longrightarrow\left(f_{1}, A_{1}\right)$ is a pair $(\varphi, \psi)$ of a morphism $\varphi: f_{0} \longrightarrow f_{1}$ in $\underline{G}$ and a 2-isomorphism $\psi: A_{0} \longrightarrow \varphi^{*} A_{1}$ in $\operatorname{Sym}_{G}^{\mathrm{PSh}}(\mathcal{G})\left(f_{0}\right)$.

The functor $p: \operatorname{Sym}_{G}(\mathcal{G}) \longrightarrow \underline{G}$ is automatically a fibration in groupoids, since it arises as the Grothendieck construction of a pseudo-presheaf of groupoids. Since Grothendieck fibrations are stable under composition [Vis05], the composite functor

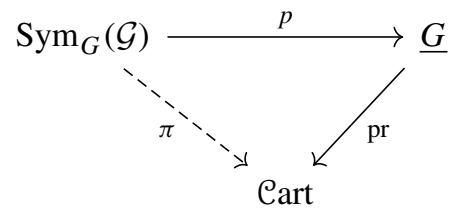

makes $\operatorname{Sym}_{G}(\mathcal{G})$ into a smooth groupoid. 
5.2. Smooth 2-groups. We would now like to establish that $\operatorname{Sym}_{G}(\mathcal{G})$ is not just a smooth groupoid, but can also be regarded as a higher group in some sense. That is, we would like to find on $\operatorname{Sym}_{G}(\mathcal{G})$ the same type of structure as we found on the bundle $\operatorname{Sym}_{G}(P) \longrightarrow$ $G$ in Sect. 3.1. Here, however, we are working inside the ambient 2-category $\mathcal{H}$, and so we will need to make precise what we mean by a group in $\mathcal{H}$. The notion of a group object in a 2-category goes back to [BL04]. The following definitions are taken from [SP11] which are strongly based on [BL04]. Let $\mathcal{C}$ be a 2-category with finite products; in particular, it has a terminal object $*$. Examples are the 2-categories $\mathcal{G r p d}$ and $\mathcal{H}$.

Definition 5.7. ([BL04]) A monoid object in $\mathcal{C}$ is a sextuple $(\mathrm{H}, \mathrm{u}, \otimes, \mathrm{a}, \mathrm{I}, \mathrm{r})$ of

- an object $\mathrm{H} \in \mathcal{C}$,

- 1-morphisms $\mathrm{u}: * \longrightarrow \mathrm{H}$ and $\otimes: \mathrm{H} \times \mathrm{H} \longrightarrow \mathrm{H}$, and

- 2-isomorphisms

$$
\begin{aligned}
& a: \otimes \circ\left(\otimes \times 1_{\mathrm{H}}\right) \longrightarrow \otimes \circ\left(1_{\mathrm{H}} \times \otimes\right), \\
& \quad \mathrm{I}: \otimes \circ\left(\mathrm{u} \times 1_{\mathrm{H}}\right) \longrightarrow 1_{\mathrm{H}}, \\
& \mathrm{r}: \otimes \circ\left(1_{\mathrm{H}} \times \mathrm{u}\right) \longrightarrow 1_{\mathrm{H}} .
\end{aligned}
$$

These data are required to satisfy a pentagon and a triangle identity; see [SP11, Definition 41].

An abelian monoid object comes with an additional 2-isomorphism $\beta: \otimes \circ \tau \longrightarrow \otimes$ satisfying the coherence conditions in [SP11, Definition 47], where $\tau: \mathrm{H} \times \mathrm{H} \longrightarrow \mathrm{H} \times \mathrm{H}$ is the interchange of factors.

Definition 5.8. A 1-morphism of monoid objects $(H, u, \otimes, a, l, r) \longrightarrow\left(H^{\prime}, u^{\prime}, \otimes^{\prime}, a^{\prime}\right.$, $\left.\mathrm{I}^{\prime}, \mathrm{r}^{\prime}\right)$ in $\mathrm{C}$ consists of a triple $\left(F_{1}, F_{\otimes}, F_{\mathrm{u}}\right)$ of

- a 1-morphism $F_{1}: \mathrm{H} \longrightarrow \mathrm{H}^{\prime}$ and

- 2-isomorphisms $F_{\otimes}: \otimes^{\prime} \circ\left(F_{1} \times F_{1}\right) \longrightarrow F_{1} \circ \otimes$ and $F_{\mathrm{u}}: \mathrm{u}^{\prime} \longrightarrow F_{1} \circ \mathrm{u}$.

These are required to satisfy the coherence conditions in [SP11, Definition 42].

Morphisms of abelian monoid objects satisfy an additional compatibility condition for the symmetries $\beta$ and $\beta^{\prime}$, which can be found in [SP11, Definition 48].

Definition 5.9. ([SP11, Definition 43]) A 2-morphism $\left(F_{1}, F_{\otimes}, F_{\mathrm{u}}\right) \longrightarrow\left(E_{1}, E_{\otimes}, E_{\mathrm{u}}\right)$ of monoid objects in $\mathcal{C}$ is a 2-morphism $\theta: F_{1} \longrightarrow E_{1}$ such that the diagrams
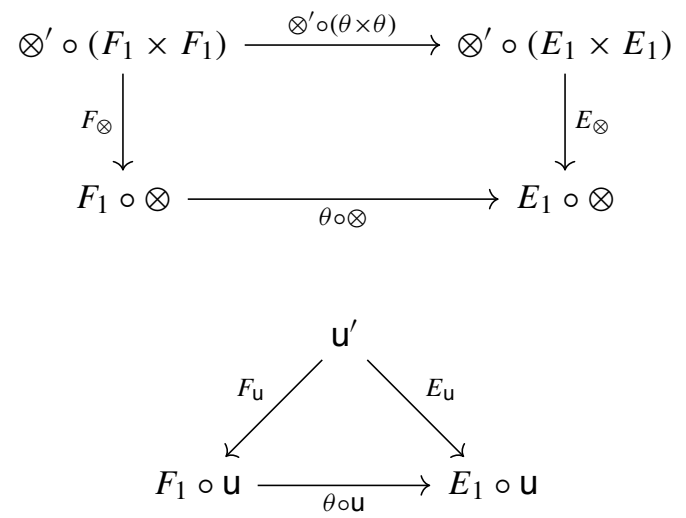

commute. 2-morphisms of abelian monoid objects are 2-morphisms of the underlying monoid objects. 
Example 5.10. A monoid object in the 2-category Cat of categories is precisely a monoidal category. Similarly, 1-morphisms and 2-morphisms between monoid objects in Cat are precisely the monoidal functors and the monoidal natural transformations, respectively. The abelian monoids in Cat are precisely the symmetric monoidal categories.

Definition 5.11 [[SP11, Definition 41]]. A group object in $\mathcal{C}$ is a monoid object $(\mathrm{H}, \mathrm{u}, \otimes$, a, $I, r)$ in $\mathcal{C}$ such that the 1-morphism

$$
\left(\otimes, \mathrm{pr}_{1}\right): \mathrm{H} \times \mathrm{H} \longrightarrow \mathrm{H} \times \mathrm{H}
$$

is (weakly) invertible. An abelian group object in $\mathcal{C}$ is an abelian monoid object whose underlying monoid object is a group object.

For $\mathcal{C}$ a 2-category with finite products, we denote the 2-category of group objects in ¿ by $2 \mathcal{G r p}(\mathcal{C})$.

Definition 5.12. The 2-category of 2-groups is $2 \mathcal{G r p}(\mathcal{G r p d})$. The 2-category of smooth 2-groups is $2 \mathcal{G r p}(\mathcal{H})$.

Both these 2-categories are enriched in groupoids. Let us examine Definition 5.12 a little more closely. Consider two objects $\pi_{\mathrm{C}}: \mathrm{C} \longrightarrow$ Cart and $\pi_{\mathrm{D}}: \mathrm{D} \longrightarrow$ Cart in $\mathcal{H}$. The product in $\mathcal{H}$ is given by the pullback in Cat:

$$
\left(C \stackrel{\pi_{C}}{\longrightarrow} \text { Cart }\right) \times\left(D \stackrel{\pi_{D}}{\longrightarrow} \text { Cart }\right)=(C \times \text { eart } D \longrightarrow \text { Cart }) .
$$

Explicitly, the category $\mathrm{C} \times$ Cart $\mathrm{D}$ has

- Objects : pairs $(c \in \mathrm{C}, d \in \mathrm{D})$ such that $\pi_{\mathrm{C}}(c)=\pi_{\mathrm{D}}(d)$.

- Morphisms : pairs $(\phi, \psi)$ of morphisms $\phi$ in C and $\psi$ in D such that $\pi_{\mathrm{C}}(\phi)=$ $\pi_{\mathrm{D}}(\psi)$.

A monoid structure on $\mathrm{C} \in \mathcal{H}$ thus allows us to multiply pairs of objects in the same fibre and pairs of morphisms that lie over the same morphism in Cart.

Example 5.13. The tensor product of line bundles turns the presheaf of groupoids of hermitean line bundles with connection $\mathrm{HLBdl}^{\nabla} \longrightarrow$ Cart into an abelian group object in $\mathcal{H}$. Similarly, for any manifold $M$ it also turns the internal hom $\left(\mathrm{HLBdl}^{\nabla}\right)^{M}$ into an abelian group object in $\mathcal{H}$.

5.3. Smooth principal 2-bundles. We shall now establish our precise notion of an extension of smooth 2-groups.

Definition 5.14. Let $\mathcal{C}$ be a 2-category with finite products, let $\left(\mathrm{H}, \mathrm{u}, \otimes_{\mathrm{H}}, \mathrm{a}, \mathrm{I}, \mathrm{r}\right)$ be a monoid object in $\mathcal{C}$, and let $\mathrm{C} \in \mathcal{C}$. A right action of $\mathrm{H}$ on $\mathrm{C}$ is a morphism $\otimes: \mathrm{C} \times \mathrm{H} \longrightarrow$ $\mathrm{C}$ in $\mathrm{C}$, together with 2-morphisms $\alpha$ and $u$ in $\mathrm{C}$ that witness the commutativity of the diagrams

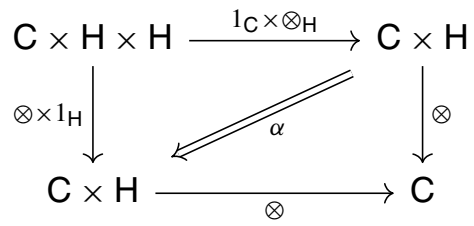

and

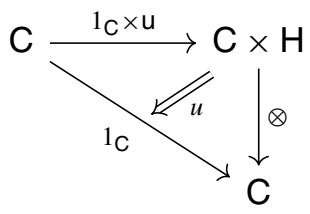

and that are coherent with respect to the 2-isomorphism a, I and r. Left actions are defined analogously. 
Example 5.15. The standard example for an action of a monoid object is that of a module category $\mathrm{C}$ over a monoidal category $\mathrm{H}$ in $\mathrm{C}=\mathrm{Cat}$.

Definition 5.16. Let $\mathcal{C}$ be a category. Suppose there are categories fibred in groupoids $\pi_{i}: \mathrm{D}_{i} \longrightarrow \mathcal{C}$, for $i=0,1$, and $\pi_{\mathrm{E}}: \mathrm{E} \longrightarrow \mathcal{C}$ over $\mathcal{C}$, and suppose there is a diagram

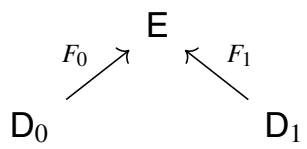

of categories fibred in groupoids over $\mathrm{C}$. The homotopy pullback $D_{0} \times \mathrm{D}_{1}^{\mathrm{h}}$ is the category with

- Objects : triples $\left(d_{0}, \eta, d_{1}\right)$, where $d_{i} \in \mathrm{D}_{i}$ and $\eta: F_{0}\left(d_{0}\right) \longrightarrow F_{1}\left(d_{1}\right)$ is an isomorphism in $\mathrm{E}$ that projects to the identity under $\pi_{\mathrm{E}}$.

- Morphisms : a morphism $\left(d_{0}, \eta, d_{1}\right) \longrightarrow\left(d_{0}^{\prime}, \eta^{\prime}, d_{1}^{\prime}\right)$ is a pair $\left(\psi_{0}, \psi_{1}\right)$ of morphisms $\psi_{i}: d_{i} \longrightarrow d_{i}^{\prime}$ such that the diagram

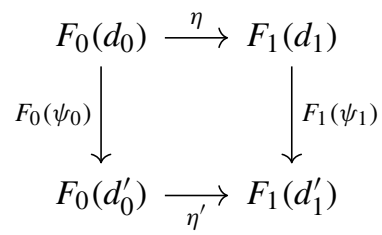

commutes in $\mathrm{E}$.

This comes with a canonical functor

$$
\begin{aligned}
\pi_{\mathrm{h}}: \mathrm{D}_{0} \times \times_{\mathrm{E}}^{\mathrm{h}} \mathrm{D}_{1} & \longrightarrow \mathcal{C} \\
\left(d_{0}, \eta, d_{1}\right) & \longmapsto \pi_{0}\left(d_{0}\right)=\pi_{1}\left(d_{1}\right) \\
\left(\psi_{0}, \psi_{1}\right) & \longmapsto \pi_{0}\left(\psi_{0}\right)=\pi_{1}\left(\psi_{1}\right),
\end{aligned}
$$

which, as we show in Appendix A, is a Grothendieck fibration in groupoids.

Definition 5.17. Let $\mathrm{H}$ be a smooth 2-group, and let $\mathrm{X} \in \mathcal{H}$ be any smooth groupoid. An $\mathrm{H}$-principal 2-bundle on $\mathrm{X}$ is an object $\mathrm{P} \in \mathcal{H}$ with a morphism $\pi: \mathrm{P} \longrightarrow \mathrm{X}$, a right action $(\otimes, \alpha)$ of $\mathrm{H}$ on $\mathrm{P}$ and a 2 -isomorphism

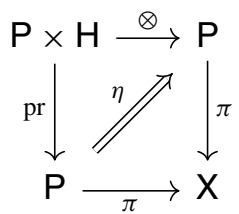

such that

(1) the functor $\pi: P \longrightarrow X$ is an essentially surjective Grothendieck fibration, 
(2) the action $(\otimes, \alpha)$ of $\mathrm{H}$ on $\mathrm{P}$ and the 2-isomorphism $\eta$ are compatible in the sense that the diagram

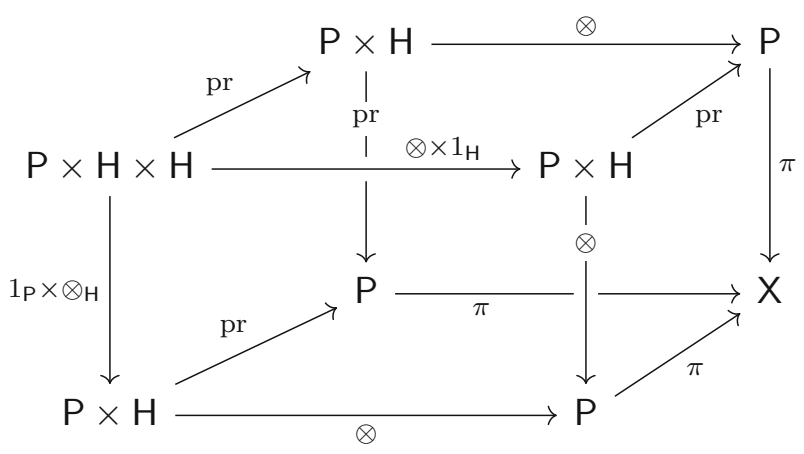

is coherent, where the front face carries the 2-isomorphism $\alpha$ that is part of the action of $\mathrm{H}$ on $\mathrm{P}$, the back, right-hand, and bottom faces carry the 2-isomorphism $\eta$, and the left-hand face commutes strictly,

(3) the composition $\mathrm{P} \times \mathrm{H} \longrightarrow \mathrm{P} \times{ }_{x} \mathrm{P} \times \mathrm{H} \longrightarrow \mathrm{P} \times{ }_{\times}^{h} \mathrm{P}$ is an equivalence, where the first functor is induced by the diagonal functor $\mathrm{P} \stackrel{\mathrm{P}}{\longrightarrow} \times \mathrm{P}$.

The first condition can be understood as demanding that $P \longrightarrow X$ has local sections (see Lemma A.1 from Appendix A). The second condition implements the property that the $\mathrm{H}$-action preserves the projection to $\mathrm{X}$ up to coherent homotopy. The third condition says that the $\mathrm{H}$-action is principal. Note that upon choosing an inverse to the equivalence $\mathrm{P} \times{ }_{X} \mathrm{P} \hookrightarrow \mathrm{P} \times{ }_{X}^{h} \mathrm{P}$, one could equivalently formulate condition (3) using strict pullbacks alone (again by Lemma A.1 from Appendix A).

In order to understand the notion of an extension of smooth 2-groups, we first need to define the kernel of a morphism of smooth 2-groups. Naively, the kernel could easily be defined as a fibre over $u$, but the resulting category will not generally be fibred in groupoids over Cart. As it turns out, the homotopy pullback does satisfy this property.

Definition 5.18. Let $p: \mathrm{H} \longrightarrow \mathrm{G}$ be a morphism of smooth 2-groups in $\mathcal{H}$. Its kernel $\operatorname{ker}^{\mathrm{h}}(p)$ is the homotopy pullback

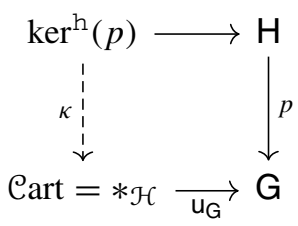

Explicitly, $\operatorname{ker}^{\mathrm{h}}(p)$ is given by

$$
\operatorname{ker}^{\mathrm{h}}(p):=* \mathcal{H} \times{ }_{\mathrm{G}}^{\mathrm{h}} \mathrm{H} .
$$

Using Definition 5.16 we can equivalently describe it as the category with objects given by pairs $(h, \eta)$ of an object $h \in \mathrm{H}$ and an isomorphism $\eta: p(h) \longrightarrow \mathrm{U}_{\mathrm{G}}\left(\pi_{\mathrm{H}}(h)\right)$ in $\mathrm{G}$. Its morphisms $\left(h_{0}, \eta_{0}\right) \longrightarrow\left(h_{1}, \eta_{1}\right)$ are given by morphisms $\zeta: h_{0} \longrightarrow h_{1}$ such that $\eta_{1} \circ p(\zeta)=u_{\mathrm{G}}\left(\pi_{\mathrm{H}}(\zeta)\right) \circ \eta_{0}$. We readily observe that the restrictions of the structure morphisms $\otimes_{\mathrm{H}}, \mathrm{a}_{\mathrm{H}}, \mathrm{l}_{\mathrm{H}}$ and $\mathrm{r}_{\mathrm{H}}$, together with the morphism $\mathrm{u}_{\mathrm{H}}$, turn $\operatorname{ker}^{\mathrm{h}}(p)$ into a 
smooth 2-group. It should also be possible to turn the strict kernel $\operatorname{ker}(p)$ into a smooth 2-group in this case, using an inverse to the equivalence $\operatorname{ker}(p) \hookrightarrow \operatorname{ker}^{\mathrm{h}}(p)$ (compare Lemma A.2 from Appendix A), but the homotopy-kernel $\operatorname{ker}^{\mathrm{h}}(p)$ carries a canonical 2-group structure, and using $\operatorname{ker}(p)$ instead of $\operatorname{ker}^{\mathrm{h}}(p)$ would make Construction 5.21 below rather cumbersome.

Lemma 5.19. In the setting of Definition 5.18, the functor $\kappa$ is a Grothendieck fibration in groupoids.

Proof. This follows directly by Lemma A.2 (1) from Appendix A.

Let $\operatorname{ker}(p)$ denote the strict pullback of the diagram Cart $\stackrel{\mathrm{u}_{\mathrm{G}}}{\longrightarrow} \mathrm{G} \stackrel{p}{\longleftarrow} \mathrm{H}$. Explicitly, it is the category with objects $h \in \mathrm{H}$ such that $p(h)=\mathrm{u}_{\mathrm{G}}\left(\pi_{\mathrm{H}}(h)\right)$ and morphisms $\zeta: h_{0} \longrightarrow h_{1}$ such that $p(\zeta)=\mathrm{u}_{\mathrm{G}}\left(\pi_{\mathrm{H}}(\zeta)\right)$. The functor $\operatorname{ker}(p) \longrightarrow$ Cart is not a Grothendieck fibration in groupoids in general. However, if $p: \mathrm{H} \longrightarrow \mathrm{G}$ is a Grothendieck fibration in groupoids, then so are the functors $\operatorname{ker}^{\mathrm{h}}(p) \longrightarrow$ Cart and $\operatorname{ker}(p) \longrightarrow$ Cart, and the canonical inclusion $\operatorname{ker}(p) \hookrightarrow \operatorname{ker}^{\mathrm{h}}(p)$ is an equivalence. The next definition is loosely modelled on [SP11, Definition 75].

Definition 5.20. Let $\mathrm{A}$ and $\mathrm{G}$ be smooth 2-groups. An extension of $\mathrm{G}$ by $\mathrm{A}$ is a pair $(F, p)$ of a morphism of smooth 2-groups $p: \mathrm{H} \longrightarrow \mathrm{G}$ that turns $\mathrm{H}$ into a $\operatorname{ker}^{\mathrm{h}}(p)$-principal 2-bundle over $\mathrm{G}$, and an equivalence of smooth 2-groups $F: \mathrm{A} \longrightarrow \operatorname{ker}^{\mathrm{h}}(p)$.

By Lemma A.2 from Appendix A, we could equivalently require $p$ to turn $\mathrm{H}$ into a $\operatorname{ker}(p)$-principal 2-bundle, but then we would need to use the non-canonical 2-group structure on $\operatorname{ker}(p)$. This essentially amounts to choosing an inverse for the equivalence $\operatorname{ker}(p) \hookrightarrow \operatorname{ker}^{\mathrm{h}}(p)$.

Our goal now is to define when an extension of smooth 2-groups is central. Again, we follow the ideas of [SP11], where the criterion for an extension of $G$ by $A$ to be central is formulated using a functor $G \longrightarrow \operatorname{Aut}(A)$ from $G$ into the automorphisms of $A$ as a 2-group; the smooth structure does not matter here. In [SP11], this functor is obtained from abstract arguments.

Construction 5.21. In our formalism, we can understand this construction as follows: consider smooth 2-groups $\mathrm{G}$ and $\mathrm{A}$, where $\mathrm{A}$ is abelian, and let $(F, p)$ be a smooth 2group extension of $\mathrm{G}$ by $\mathrm{A}$, with morphism $p: \mathrm{H} \longrightarrow \mathrm{G}$. Then $\mathrm{A}$ is abelian if and only if $\operatorname{ker}^{\mathrm{h}}(p)$ is abelian, which is true if and only if $\operatorname{ker}(p)$ is abelian (since the 2-group structure induces Picard groupoid structures on the fibres of these smooth 2-groups, where $F$ induces monoidal equivalences). Fix an arbitrary Cartesian space $c \in$ Cart. Let $\operatorname{Aut}\left(\operatorname{ker}^{\mathrm{h}}(p)_{\mid c}\right)$ denote the Picard groupoid of monoidal autoequivalences of the fibre $\operatorname{ker}^{\mathrm{h}}(p)_{\mid c}$ of $\operatorname{ker}^{\mathrm{h}}(p)$ over $c$. Note that we do not claim that the Picard groupoids Aut $\left(\operatorname{ker}^{\mathrm{h}}(p)_{\mid c}\right)$ assemble into a smooth 2-group (though it might be possible to achieve this). We claim that there is a functor $\mathrm{G}_{\mid c} \longrightarrow \operatorname{Aut}\left(\operatorname{ker}^{\mathrm{h}}(p)_{\mid c}\right)$ which is canonical up to unique natural isomorphism.

Let $(\cdot)^{\vee}: \mathrm{H}_{\mid c} \longrightarrow \mathrm{H}_{\mid c}$ denote a choice of functorial inverse in $\mathrm{H}_{\mid c}$. This can always be enhanced to a functorial choice of adjoint inverse, i.e. a functor $k \longmapsto\left(k \vee, \mathrm{ev}_{k}, \operatorname{coev}_{k}\right)$ that maps $k$ to a triple of a dual object $k^{\vee}$, and duality morphisms (which are isomorphisms in this case) $\mathrm{ev}_{k}: k \otimes_{\mathrm{H}} k^{\vee} \longrightarrow \mathrm{u}_{\mathrm{H}}(c)$ and $\operatorname{coev}_{k}: \mathrm{u}_{\mathrm{H}}(c) \longrightarrow k^{\vee} \otimes_{\mathrm{H}} k$ which satisfy the triangle identities. The functor $(\cdot)^{\vee}$ acts on morphisms $\psi: k \longrightarrow k^{\prime}$ by taking the dual of $\psi^{-1}$ with respect to the chosen duality data on $k$ and $k^{\vee}$. This enhancement can be achieved by choosing an adjoint inverse for the equivalence of categories $\left(\otimes_{\mathrm{H}}, \mathrm{pr}_{1}\right)_{\mid c}: \mathrm{H}_{\mid c} \times \mathrm{H}_{\mid c} \longrightarrow \mathrm{H}_{\mid c} \times \mathrm{H}_{\mid c}$ (which is always possible). 
To an object $k \in \mathrm{H}_{\mid c}$ we associate the functor

$$
\begin{aligned}
\operatorname{Ad}_{k}: \operatorname{ker}^{\mathrm{h}}(p)_{\mid c} & \longrightarrow \operatorname{ker}^{\mathrm{h}}(p)_{\mid c} \\
(h, \varphi) & \longmapsto\left(k \otimes_{\mathrm{H}} h \otimes_{\mathrm{H}} k^{\vee}, \varphi_{k}\right) \\
\left(\zeta:\left(h_{0}, \varphi_{0}\right) \longrightarrow\left(h_{1}, \varphi_{1}\right)\right) & \longmapsto 1_{k} \otimes_{\mathrm{H}} \zeta \otimes_{\mathrm{H}} 1_{k^{\vee}},
\end{aligned}
$$

where the morphism $\varphi_{k}$ is the composition

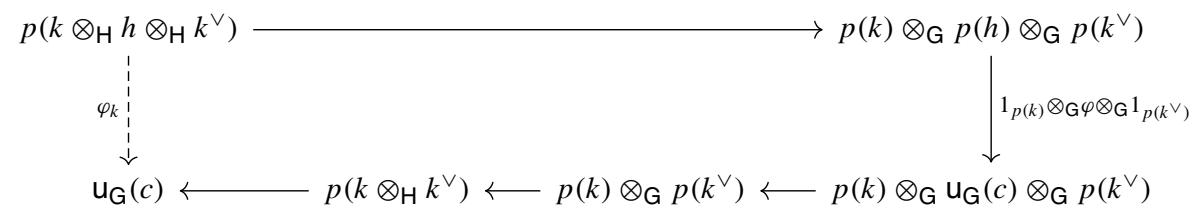

Given another object $k^{\prime} \in \mathrm{H}_{\mid c}$ such that $p(k)=p\left(k^{\prime}\right)$ in $\mathrm{G}$, the principality condition implies that there exists an object $(b, \beta) \in \operatorname{ker}^{\mathrm{h}}(p)$ and an isomorphism $\psi: k^{\prime} \longrightarrow$ $k \otimes_{\mathrm{H}} b$. Since $\operatorname{ker}^{\mathrm{h}}(p)$ is abelian, this induces an isomorphism

$$
\alpha_{k, k^{\prime}}:\left(1_{k} \times \mathrm{ev}_{b} \times 1_{k^{\vee}}\right) \circ\left(\psi \times 1_{h} \times \psi^{\vee}\right): \operatorname{Ad}_{k^{\prime}}(h) \longrightarrow \operatorname{Ad}_{k}(h) .
$$

By the functoriality of $(\cdot)^{\vee}$, any other choice of $(b, \beta)$ and $\psi$ yields the same isomorphism $\operatorname{Ad}_{k^{\prime}}(h) \longrightarrow \operatorname{Ad}_{k}(h)$ in this way. Furthermore, this isomorphism is natural in $k$ and $h$ by the functoriality of $\otimes_{\mathrm{H}}$ and $(\cdot)^{\vee}$. That is, the pair $(\mathrm{Ad}, \alpha)$ defines an object

$$
(\operatorname{Ad}, \alpha)_{c} \in \operatorname{holim}^{\operatorname{Grpd}} \underline{2 \operatorname{Grp}}\left(\mathrm{H}_{\mid c}^{[\bullet]}, \operatorname{Aut}\left(\operatorname{ker}^{\mathrm{h}}(p)_{\mid c}\right)\right),
$$

where $\mathrm{H}_{\mid c}^{[\bullet]} \longrightarrow \mathrm{G}_{\mid c}$ denotes the Čech nerve of the functor $p_{\mid c}$. As we show in the proof of Proposition A.3 in Appendix A, any choice of preimages of the objects $g \in \mathrm{G}_{\mid c}$ under $p_{\mid c}$ now induces a functor $\mathrm{G}_{\mid c} \longrightarrow \operatorname{Aut}\left(\operatorname{ker}^{\mathrm{h}}(p)_{\mid c}\right)$ from these data. Moreover, any other choice of such preimages will induce a canonical natural isomorphism of functors. Hence we obtain a well-defined isomorphism class of functors, which we denote by

$$
[\operatorname{Ad}, \alpha]_{c} \in \pi_{0}\left(\underline{\operatorname{2Grp}}\left(\mathrm{G}_{\mid c}, \operatorname{Aut}\left(\operatorname{ker}^{\mathrm{h}}(p)_{\mid c}\right)\right)\right) .
$$

This class allows us to state when a smooth 2-group extension is central.

Definition 5.22. Let $(F, p)$ be an extension of a smooth 2-group $\mathrm{G}$ by a smooth 2-group A. Then $(F, p)$ is central if $\mathrm{A}$ is abelian, and for every $c \in$ Cart the isomorphism class $[\mathrm{Ad}, \alpha]_{c}$ agrees with the isomorphism class of the trivial 2-group morphism $\mathrm{G}_{\mid c} \longrightarrow$ $\operatorname{Aut}\left(\operatorname{ker}^{\mathrm{h}}(p)_{\mid c}\right)$.

5.4. Global description of the 2-group extension. We shall now apply the general considerations of Sects. 5.2 and 5.3 to the smooth groupoid $\operatorname{Sym}_{G}(\mathcal{G})$ constructed in Sect. 5.1.

Theorem 5.23. The functor $\pi: \operatorname{Sym}_{G}(\mathcal{G}) \longrightarrow$ Cart is a smooth 2-group. 
Proof. First we show that $\operatorname{Sym}_{G}(\mathcal{G})$ carries the structure of a monoid object in $\mathcal{H}$. The terminal object $* \mathcal{H} \in \mathcal{H}$ is the identity functor $1_{\text {Cart }}$ : Cart $\longrightarrow$ Cart. We start by defining the 1-morphism $\mathrm{u}: * \mathcal{H} \longrightarrow \operatorname{Sym}_{G}(\mathcal{G})$; it is the functor that assigns to every object $c \in$ Cart the object $\left(e_{c}, \operatorname{pr}_{M}^{*} 1_{\mathcal{G}}\right) \in \operatorname{Sym}_{G}(\mathcal{G})$, where $e_{c}: c \longrightarrow G$ is the constant map at the identity object $e \in G$.

Next we define the 1-morphism

$$
\otimes: \operatorname{Sym}_{G}(\mathcal{G}) \times \text { eart } \operatorname{Sym}_{G}(\mathcal{G}) \longrightarrow \operatorname{Sym}_{G}(\mathcal{G})
$$

in the following way: consider two arbitrary objects $\left(f_{0}, A_{0}\right),\left(f_{1}, A_{1}\right) \in \operatorname{Sym}_{G}(\mathcal{G})$ in the same fibre of $\pi: \operatorname{Sym}_{G}(\mathcal{G}) \longrightarrow$ Cart, i.e. $f_{0}, f_{1}$ are defined over the same object $c \in$ Cart. We define the map $\left(1, \Phi_{f_{0}}\right)$ as the composition

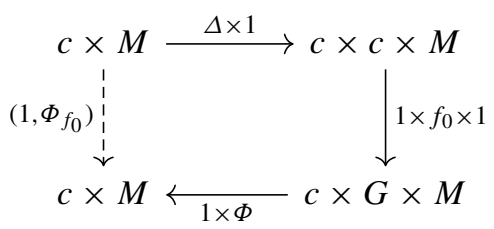

Observe that

$$
\begin{gathered}
\operatorname{pr}_{M} \circ\left(1, \Phi_{f_{0}}\right)=\Phi_{f_{0}}, \quad \Phi_{f_{1}} \circ\left(1, \Phi_{f_{0}}\right)=\Phi_{f_{1} f_{0}} \\
\quad \text { and } \quad\left(1, \Phi_{f_{1}}\right) \circ\left(1, \Phi_{f_{0}}\right)=\left(1, \Phi_{f_{1} f_{0}}\right),
\end{gathered}
$$

where the second and third identities use the fact that $\Phi$ is a group action. Thus we can form the 1-morphism

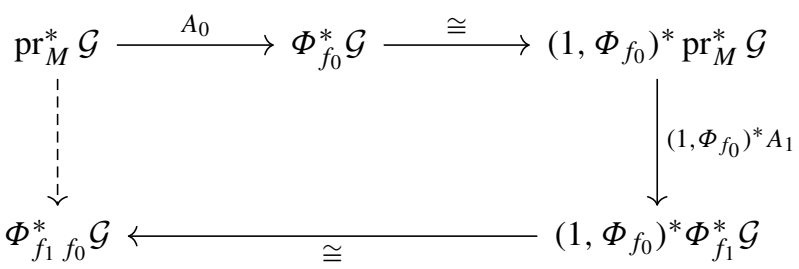

The solid unlabelled arrows are canonical isomorphisms that stem from the fact that BGrb is a (pre)sheaf of 2-categories on the category of manifolds $\mathcal{M f d}$ [Wal07b, NS11]. By a slight abuse of notation, we denote the composite morphism by $\left(1, \Phi_{f_{0}}\right)^{*} A_{1} \circ A_{0}$. Then we set

$$
\left(f_{1}, A_{1}\right) \otimes\left(f_{0}, A_{0}\right):=\left(f_{1} f_{0},\left(1, \Phi_{f_{0}}\right)^{*} A_{1} \circ A_{0}\right),
$$

and analogously on 2-isomorphisms. The associator and unitors are readily obtained from those in the sheaf of 2-categories BGrb. The coherence conditions in BGrb imply that $\operatorname{Sym}_{G}(\mathcal{G})$, endowed with the multiplication and coherence morphisms defined here, is a monoid object in $\mathcal{H}$.

Now we show that $\operatorname{Sym}_{G}(\mathcal{G})$ is in fact a group object in $\mathcal{H}$. Set

$$
(f, A)^{-1}:=\left(f^{-1},\left(1, \Phi_{f^{-1}}\right)^{*} A^{-1}\right)
$$

and analogously on morphisms, where $f^{-1}$ denotes the composition of the map $f: c \longrightarrow$ $G$ with the inversion map in the group $G$. It follows from the properties (5.24) of $\Phi_{(\cdot)}$ 
that this provides a functorial (two-sided) inverse object with respect to the 1-morphism $\otimes$, and hence shows that the morphism

$$
\left(\otimes, \operatorname{pr}_{1}\right): \operatorname{Sym}_{G}(\mathcal{G}) \times \operatorname{Cart}_{\operatorname{Cym}_{G}}(\mathcal{G}) \longrightarrow \operatorname{Sym}_{G}(\mathcal{G}) \times \operatorname{eart}_{\operatorname{Sym}}(\mathcal{G})
$$

is an equivalence in $\mathcal{H}$ (where the product is taken in $\mathcal{H}$ ). Thus $\operatorname{Sym}_{G}(\mathcal{G}) \longrightarrow$ Cart is indeed a group object in $\mathcal{H}$.

Theorem 5.27. There is a smooth 2-group extension

$$
1 \longrightarrow \operatorname{HLBdl}^{M} \stackrel{\iota}{\longrightarrow} \operatorname{Sym}_{G}(\mathcal{G}) \stackrel{p}{\longrightarrow} \underline{G} \longrightarrow 1
$$

where we abbreviate $* \mathcal{H}=$ Cart by 1 .

Proof. The projection functor pr: $\underline{G} \longrightarrow$ Cart is a smooth 2 -group via $\otimes_{G}: \underline{G} \times$ Cart $\underline{G} \longrightarrow \underline{G}$ defined by

$$
\left(f_{1}: c \longrightarrow G\right) \otimes_{G}\left(f_{0}: c \longrightarrow G\right)=\left(f_{1} f_{0}: c \longrightarrow G\right) .
$$

It is evident from (5.25) that $p: \operatorname{Sym}_{G}(\mathcal{G}) \longrightarrow \underline{G}$ is a morphism of smooth 2-groups. It is a Grothendieck fibration in groupoids by construction, and it is surjective on objects since $G$ is connected (as we have argued at the beginning of this section).

Next we define the morphism $\iota: \operatorname{HLBdl}^{M} \longrightarrow \operatorname{Sym}_{G}(\mathcal{G})$ in $\mathcal{H}$. Over a Cartesian space $c \in$ Cart, it is simply the canonical inclusion

$$
\operatorname{HLBdl}(c \times M) \longrightarrow \operatorname{BGrb}(c \times M)\left(\operatorname{pr}_{M}^{*} \mathcal{G}, \Phi_{e_{c}}^{*} \mathcal{G}\right)=\operatorname{BGrb}(c \times M)\left(\operatorname{pr}_{M}^{*} \mathcal{G}, \operatorname{pr}_{M}^{*} \mathcal{G}\right)
$$

Here $e_{c}: c \longrightarrow G$ is the constant map at the unit element of $G$. Since the inclusion of line bundles into morphisms of bundle gerbes strictly maps the tensor product to the composition [Wal07b, Bun17], we readily find that $\iota$ is a morphism of smooth 2-groups.

To see that (5.28) is an extension of smooth 2-groups, we first show that the inclusion $\iota$ is an equivalence $\mathrm{HLBdl}^{M} \longrightarrow \operatorname{ker}^{\mathrm{h}}(p)$. By Lemma A.2 from Appendix A and the fact that $p: \operatorname{Sym}_{G}(\mathcal{G}) \longrightarrow \underline{G}$ is a Grothendieck fibration in groupoids, it follows that the canonical inclusion $\operatorname{ker}(\bar{p}) \hookrightarrow \operatorname{ker}^{\mathrm{h}}(p)$ is an equivalence. Consequently, it suffices to show that $\iota$ induces an equivalence $\operatorname{HLBdl}^{M} \longrightarrow \operatorname{ker}(p)$. Over an object $c \in$ Cart, the fibre of $\operatorname{ker}(p)$ consists of the automorphism groupoid of $\operatorname{pr}_{M}^{*} \mathcal{G} \in \mathrm{BGrb}(c \times M)$. It is well-known [Wal07b] that the inclusion $\operatorname{HLBdl}(c \times M) \longrightarrow \mathrm{BGrb}(c \times M)$ given by $L \longmapsto L \otimes 1_{\mathrm{pr}_{M}^{*} \mathcal{G}}$ is an equivalence of groupoids.

To see that the functor $p: \operatorname{Sym}_{G}(\mathcal{G}) \longrightarrow \underline{G}$ is an $\mathrm{HLBdl}^{M}$-principal 2-bundle (see Definition 5.17), it now suffices to show that the functor

$$
\begin{aligned}
(1, \alpha): \operatorname{Sym}_{G}(\mathcal{G}) \times \operatorname{Cart}_{\operatorname{aLBdl}} \operatorname{HL}^{M} & \longrightarrow \operatorname{Sym}_{G}(\mathcal{G}) \times_{\underline{G}} \operatorname{Sym}_{G}(\mathcal{G}) \\
((f, A), L) & \longmapsto((f, A),(f, A \otimes L))
\end{aligned}
$$

is an equivalence in $\mathcal{H}$, where we have written out the product in $\mathcal{H}$ as the fibre product over Cart. Observe that by the equivalence $\operatorname{HLBdl}^{M} \longrightarrow \operatorname{ker}^{\mathrm{h}}(p)$, it is enough to consider the action of $\mathrm{HLBdl}^{M}$, and since $\underline{G}$ has discrete fibres, i.e. the fibres have no non-identity morphisms, there is an identity $\operatorname{Sym}_{G}(\mathcal{G}) \times{ }_{G}^{\mathrm{h}} \operatorname{Sym}_{G}(\mathcal{G})=\operatorname{Sym}_{G}(\mathcal{G}) \times_{\underline{G}}$ $\operatorname{Sym}_{G}(\mathcal{G})$, and hence we can work with the strict pullback instead of the homotopy pullback. 
Since both sides are fibred over Cart, it suffices to show that this functor is an equivalence on all fibres [Vis05, Proposition 3.36]. Thus we fix an object $c \in$ Cart. We need to check that the functor

$$
\begin{aligned}
(1, \alpha)_{\mid c}: \operatorname{Sym}_{G}(\mathcal{G})_{\mid c} \times \operatorname{HLBdl}(c \times M) & \longrightarrow \operatorname{Sym}_{G}(\mathcal{G})_{\mid c} \times_{\underline{G}_{\mid c}} \operatorname{Sym}_{G}(\mathcal{G})_{\mid c} \\
((f, A), L) & \longmapsto((f, A),(f, A \otimes L))
\end{aligned}
$$

is an equivalence. By construction, both sides decompose into coproducts

$$
\begin{aligned}
& \operatorname{Sym}_{G}(\mathcal{G})_{\mid c} \times \operatorname{HLBdl}(c \times M) \\
& \quad=\coprod_{f: c \longrightarrow G} \operatorname{BGrb}(c \times M)\left(\operatorname{pr}_{M}^{*} \mathcal{G}, \Phi_{f}^{*} \mathcal{G}\right) \times \operatorname{HLBdl}(c \times M)
\end{aligned}
$$

and

$$
\begin{aligned}
& \operatorname{Sym}_{G}(\mathcal{G})_{\mid c} \times_{\underline{G}_{\mid c}} \operatorname{Sym}_{G}(\mathcal{G})_{\mid c} \\
& =\coprod_{f: c \longrightarrow G} \operatorname{BGrb}(c \times M)\left(\operatorname{pr}_{M}^{*} \mathcal{G}, \Phi_{f}^{*} \mathcal{G}\right) \times \operatorname{BGrb}(c \times M)\left(\operatorname{pr}_{M}^{*} \mathcal{G}, \Phi_{f}^{*} \mathcal{G}\right),
\end{aligned}
$$

so the functor $(1, \alpha)_{\mid c}$ decomposes into functors

$$
\begin{aligned}
(1, \alpha)_{\mid f}: \operatorname{BGrb}(c \times M) & \left(\operatorname{pr}_{M}^{*} \mathcal{G}, \Phi_{f}^{*} \mathcal{G}\right) \times \operatorname{HLBdl}(c \times M) \\
& \longrightarrow \operatorname{BGrb}(c \times M)\left(\operatorname{pr}_{M}^{*} \mathcal{G}, \Phi_{f}^{*} \mathcal{G}\right) \times \operatorname{BGrb}(c \times M)\left(\operatorname{pr}_{M}^{*} \mathcal{G}, \Phi_{f}^{*} \mathcal{G}\right)
\end{aligned}
$$

This functor acts as the identity on the first factor and as the standard action of line bundles on isomorphisms of bundle gerbes in the second factor. Thus $(1, \alpha)_{\mid f}$ is an equivalence since on any manifold $X$, the category of 1-isomorphisms between any given bundle gerbes is a torsor category over $\operatorname{HLBdl}(X)$ with respect to this action [Wal07b].

Proposition 5.29. If $G$ acts non-trivially on $M$, then the extension (5.28) is not central.

Proof. This follows readily from the explicit forms (5.25) and (5.26) of the product and the inverse in $\operatorname{Sym}_{G}(\mathcal{G})$, together with the fact that composition of morphisms of bundle gerbes is compatible with tensoring by line bundles. Explicitly, given $(f, A) \in$ $\operatorname{Sym}_{G}(\mathcal{G})_{\mid c}$ and $L \in \operatorname{HLBdl}(c \times M)$ we find

$$
(f, A) \otimes \iota(L) \otimes(f, A)^{-1} \cong\left(e_{c}, \Phi_{f}^{*} L\right)=\iota\left(\Phi_{f}^{*} L\right) .
$$

Hence $(\operatorname{Ad}, \alpha)_{c}(f)(L) \cong \Phi_{f}^{*} L$. Observe that since $\underline{G}$ has discrete fibres, we have $\operatorname{ker}^{\mathrm{h}}(p)=\operatorname{ker}(p)$, and by the equivalence $\operatorname{HLBdl}^{M} \longrightarrow \operatorname{ker}(p)$ it is sufficient to consider the adjoint action on the smooth 2-group $\mathrm{HLBdl}^{M}$ here.

Let $c=*$, so that the data $f$ corresponds to an element $g \in G$. Assume that the extension (5.28) is central. Then, by Construction 5.21 and Definition 5.22, there is an isomorphism $\varphi:(\operatorname{Ad}, \alpha)_{*} \longrightarrow 1_{\mathrm{HLBdl}(M)}$ of morphisms of 2-groups $G \longrightarrow \operatorname{Aut}(\operatorname{HLBdl}(M))$. Let $I \in \operatorname{HLBdl}(M)$ denote the trivial line bundle, and let $\psi: I \longrightarrow I$ be any automorphism, i.e. any $\mathrm{U}(1)$-valued function on $M$. The naturality of $\varphi$ then implies, in particular, that the diagram

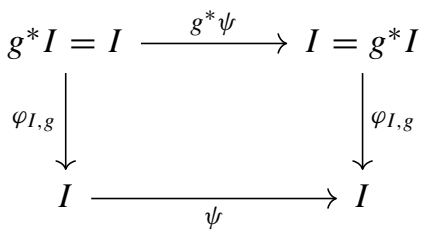


commutes. But this is equivalent to the identity $\psi=g^{*} \psi$ for any $g \in G$ and $\psi: M \longrightarrow$ $\mathrm{U}(1)$, which is a contradiction if the $G$-action on $M$ is non-trivial.

We now obtain an action of $\operatorname{Sym}_{G}(\mathcal{G})$ on $\mathcal{G}$ in the following sense: let $\widehat{\mathcal{G}} \in \mathcal{H}$ denote the category fibred in groupoids over Cart which is defined as follows. Consider the presheaf of groupoids on $\underline{M}$ that assigns to $f: c \longrightarrow M$ the category $\operatorname{BGrb}(c)\left(\mathcal{I}, f^{*} \mathcal{G}\right)$. Then $q: \widehat{\mathcal{G}} \longrightarrow \underline{M}$ is obtained by applying the Grothendieck construction to this presheaf. The action of $\operatorname{Sym}_{G}(\mathcal{G})$ on $\mathcal{G}$ is then the morphism of categories over $\underline{G \times M} \cong$ $\underline{G} \times \underline{M}$ obtained through the diagram

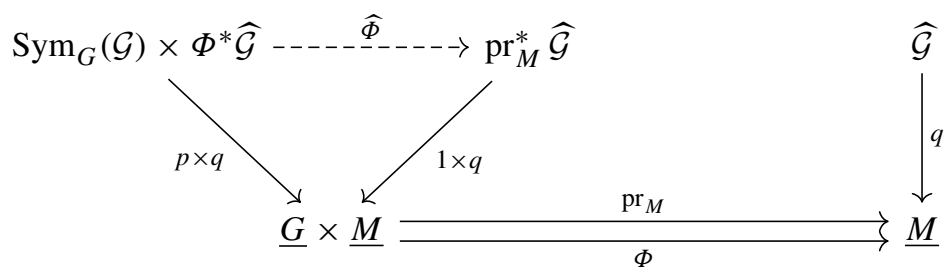

where we suppress pullbacks and denote by $\operatorname{pr}_{M}, \Phi: \underline{G} \times \underline{M} \longrightarrow \underline{M}$ the functors induced from the smooth maps $\operatorname{pr}_{M}, \Phi$ via postcomposition. The functor $\widehat{\Phi}$ sends an object $(\mathcal{A}, \mathcal{J}) \in \operatorname{Sym}_{G}(\mathcal{G})_{\mid c} \times \widehat{\mathcal{G}}_{\mid f}$ to the composition $\Delta^{*}\left(1_{c} \times f\right)^{*}(\mathcal{A}, \mathcal{J})$, where $\Delta: c \longrightarrow c \times c$ is the diagonal map.

The construction $\operatorname{Sym}_{G}$ is 2-functorial: let $E: \mathcal{G} \longrightarrow \mathcal{G}^{\prime}$ be a 1-isomorphism of bundle gerbes. Pick an adjoint inverse $E^{\vee}$. The 1-isomorphism $E$ induces a 1-isomorphism of smooth 2-groups

$$
\begin{aligned}
\widehat{E}: \operatorname{Sym}_{G}(\mathcal{G}) & \longrightarrow \operatorname{Sym}_{G}\left(\mathcal{G}^{\prime}\right) \\
\left(f: c \longrightarrow G, A: \operatorname{pr}_{M}^{*} \mathcal{G} \longrightarrow \Phi_{f}^{*} \mathcal{G}\right) & \longmapsto\left(f, \operatorname{pr}_{M}^{*} \mathcal{G}^{\prime} \stackrel{\operatorname{pr}_{M}^{*} E^{\vee}}{\longrightarrow} \operatorname{pr}_{M}^{*} \mathcal{G} \stackrel{A}{\longrightarrow} \Phi_{f}^{*} \mathcal{G} \stackrel{\Phi_{f}^{*} E}{\longrightarrow} \Phi_{f}^{*} \mathcal{G}^{\prime}\right) .
\end{aligned}
$$

Let $E, E^{\prime}: \mathcal{G} \longrightarrow \mathcal{G}^{\prime}$ be 1-isomorphisms and $\eta: E \longrightarrow E^{\prime}$ a 2-isomorphism. We construct a smooth 2-isomorphism $\widehat{\eta}: \widehat{E} \longrightarrow \widehat{E}^{\prime}$ whose component at an object $(f, A)$ of $\operatorname{Sym}_{G}(\mathcal{G})$ is given by

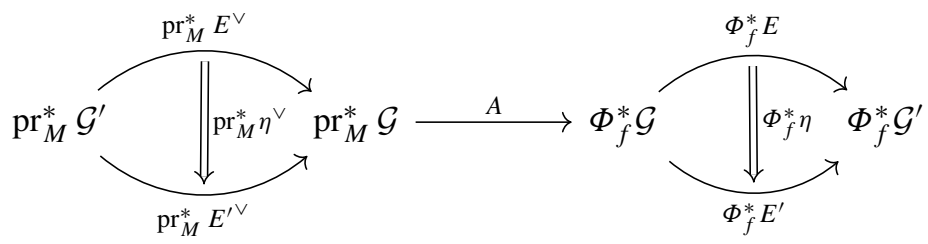

5.5. Descent description of the 2-group extension. We can describe the smooth 2-group $\operatorname{Sym}_{G}(\mathcal{G})$ in a way analogously to Sect. 3.3; that is, we can construct $\operatorname{Sym}_{G}(\mathcal{G})$ in terms of descent data for the path fibration $P_{0} G \longrightarrow G$ and the parallel transport on $\mathcal{G}$ introduced in Sect. 4. However, for a bundle gerbe $\mathcal{G}$ this construction is more involved compared to the case of a principal bundle $P$. In particular, we need to replace the associated bundle construction $\left(P_{0} G \times \mathrm{Gau}(P)\right) / \sim$ of $\mathcal{L}_{G}$ (cf. Section 3.3) by a homotopy-coherent version. Once established, the descent presentation of $\operatorname{Sym}_{G}(\mathcal{G})$ 
allows us to study and compute this smooth 2-group very explicitly in certain situations, as we demonstrate in Sect. 6.

Recalling the notation of Sect. 3.3, let $\mathcal{G} \in \operatorname{BGrb}^{\nabla}(M)$ be a bundle gerbe with connection on $M$. Using the smooth map (3.8) we obtain a diffeological hermitean line bundle

$$
\mathrm{L}^{\dashv}:=(L \Phi)^{*}(\mathcal{T} \mathcal{G}) \longrightarrow\left(P_{0} G\right)^{[2]} \times M .
$$

This object is completely analogous to (3.8) when one views the holonomy of a line bundle $L$ on $M$ as the transgression of $L$ to the loop space $L M$, and subsequently the transgression line bundle $\mathcal{T} \mathcal{G}$ as the holonomy of the bundle gerbe $\mathcal{G}$ on $M$ (cf. Section 4.4). In the adjoint picture, we can interpret $\mathrm{L}^{-}$as a smooth assignment of a line bundle with connection $\mathrm{L}_{(\gamma, \alpha)}$ on $M$ to each pair of based paths $(\gamma, \alpha) \in\left(P_{0} G\right)^{[2]}$.

Consider the simplicial diffeological space $\left(P_{0} G\right)^{[\bullet]} \times M$ with face maps

$$
d_{i}:\left(P_{0} G\right)^{[n]} \times M \longrightarrow\left(P_{0} G\right)^{[n-1]} \times M
$$

for $0 \leq i \leq n-1$ defined by deleting the $i$ th entry of $\left(P_{0} G\right)^{[n]}$. The fusion product $\lambda$ on the transgression line bundle $\mathcal{T} \mathcal{G}$ over the loop space $L M$ induces an isomorphism

$$
(L \Phi)^{*} \lambda: d_{0}^{*} \mathrm{~L}^{\dashv} \otimes d_{2}^{*} \mathrm{~L}^{\dashv} \longrightarrow d_{1}^{*} \mathrm{~L}^{\dashv}
$$

of hermitean line bundles over $\left(P_{0} G\right)^{[3]} \times M$, which is coherent over $\left(P_{0} G\right)^{[4]} \times M$.

Remark 5.32. In an adjoint fashion, the hermitean line bundle $\mathrm{L}^{\dashv}$ on $\left(P_{0} G\right)^{[2]} \times M$ from (5.30) can be seen as a morphism of smooth groupoids $\mathrm{L}:\left(P_{0} G\right)^{[2]} \longrightarrow \mathrm{HLBdl}^{M}$ (under the equivalence of diffeological vector bundles and morphisms to HLBdl following from [Bun20a, Thm. 4.8]). In this picture, the coherent isomorphism $(L \Phi)^{*} \lambda$ from (5.31) corresponds to a coherent isomorphism $d_{0}^{*} \mathrm{~L} \otimes d_{2}^{*} \mathrm{~L} \longrightarrow d_{1}^{*} \mathrm{~L}$ of morphisms $\left(P_{0} G\right)^{[3]} \longrightarrow \mathrm{HLBdl}^{M}$. In this sense, $\left(\mathrm{L}^{-},(L \Phi)^{*} \lambda\right)$ represent a smooth $\mathrm{HLBdl}^{M_{-}}$ valued Čech 1-cocycle on $G$ with respect to the Čech nerve of the subduction $P_{0} G \longrightarrow$ $G$. Note that this nicely fits the formalism for higher principal bundles with not-necessarily strict structure groups—-such as $\mathrm{HLBdl}^{M}$ — from [NSS15].

Definition 5.33. Let $c \in$ Cart be a Cartesian space and $f: c \longrightarrow G$ a smooth map. We define a category $\mathfrak{D e s}_{\mathrm{L}}^{\mathrm{PSh}}(f)$ with

- Objects : pairs $(J, J)$, where $J \in \operatorname{HLBdl}\left(f^{*} P_{0} G \times M\right)$ and where $J$ is an isomorphism of hermitean line bundles

$$
J: d_{1}^{*} J \longrightarrow\left(\widehat{f}^{[2]} \times 1\right)^{*} \mathrm{~L}^{\dashv} \otimes d_{0}^{*} J
$$

over $\left(f^{*} P_{0} G\right)^{[2]} \times M$, where $\widehat{f}^{[n]}:\left(f^{*} P_{0} G\right)^{[n]} \longrightarrow P_{0} G^{[n]}$ is the canonical map induced by the pullback of the subduction $P_{0} G \longrightarrow G$ along $f$. These data are required to satisfy the compatibility condition that

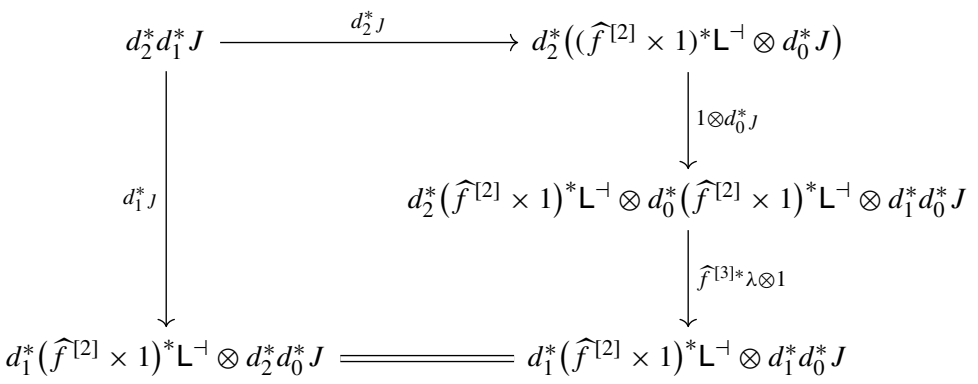


is a commutative diagram in $\operatorname{HLBdl}\left(\left(f^{*} P_{0} G\right)^{[3]} \times M\right)$, where we use the simplicial relations.

- Morphisms : a morphism $(J, J) \longrightarrow\left(J^{\prime}, J^{\prime}\right)$ is an isomorphism $\psi: J \longrightarrow J^{\prime}$ such that

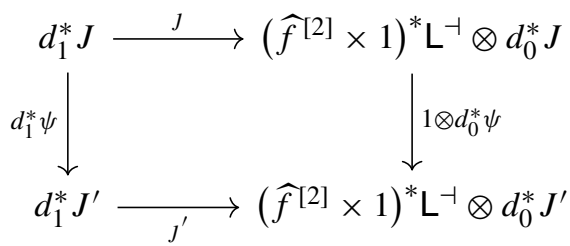

is a commutative diagram in $\operatorname{HLBdl}\left(\left(f^{*} P_{0} G\right)^{[2]} \times M\right)$.

Pullbacks of morphisms of bundle gerbes turns the assignment $(f: c \longrightarrow G) \longmapsto$ $\mathfrak{D e s}_{\mathrm{L}}^{\mathrm{PSh}}(f)$ into a presheaf of groupoids on $\underline{G}$. (This is actually even a sheaf of groupoids, but we will not need this fact here.) Applying the Grothendieck construction, we obtain a category fibred in groupoids over $\underline{G}, p_{\mathrm{L}}: \mathfrak{D e s} \longrightarrow \underline{G}$, and composing with the canonical projection functor $\mathrm{pr}: \underline{G} \longrightarrow$ Cart we obtain a category fibred in groupoids over Cart

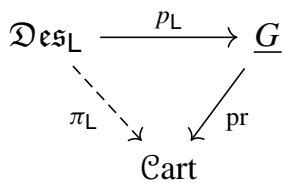

which defines a smooth groupoid $\mathfrak{D} \mathfrak{e} \mathfrak{S}_{\mathrm{L}}$.

Proposition 5.35. The functor $\pi_{\mathrm{L}}: \mathfrak{D e s} \mathrm{L} \longrightarrow$ Cart is a smooth 2-group.

Proof. Let $\left(f_{0}, J_{0}, J_{0}\right),\left(f_{1}, J_{1}, J_{1}\right) \in \mathfrak{D e s}$ be two objects, where $\left(J_{i}, J_{i}\right)$ lies in the fibre over a smooth map $f_{i}: c \longrightarrow G$ for $i=0,1$. The product $\left(f_{1}, J_{1}, J_{1}\right) \otimes\left(f_{0}, J_{0}, J_{0}\right)$ is defined as follows. First, observe that it should lie in the fibre of $\mathfrak{D} \mathfrak{e} \mathfrak{L}$ over the pointwise product map $f_{1} f_{0}: c \longrightarrow G, u \longmapsto f_{1}(u) f_{0}(u)$. Consider the smooth map

$$
\begin{aligned}
F: m^{*} P_{0} G \times_{G \times G} \operatorname{pr}_{1}^{*} P_{0} G \times_{G \times G} \operatorname{pr}_{2}^{*} P_{0} G & \longrightarrow P_{\partial \Delta^{2}} G \\
\left(\gamma_{10}, \gamma_{1}, \gamma_{0}\right) & \longmapsto F\left(\gamma_{10}, \gamma_{1}, \gamma_{0}\right)=\left(\gamma_{10}, \gamma_{1} \gamma_{0}(1), \gamma_{0}\right),
\end{aligned}
$$

where $m: G \times G \longrightarrow G$ is the multiplication of $G$, and $\mathrm{pr}_{1}$ and $\mathrm{pr}_{2}$ are the projections to the first and second factors of $G \times G$. Let us denote by $\Phi_{\mathrm{ev}_{1}}$ the composition $P_{0} G \times$ $M \longrightarrow G \times M \longrightarrow M$, where the first map evaluates a based path at its end point and the second map is the action $\Phi$ of $G$ on $M$. The pair $\left(f_{1}, f_{0}\right)$ defines a map $c \longrightarrow G \times G$. Let s: $P_{\partial \Delta^{2}} G \longrightarrow L G$ be the map defined in (4.10), and let (by a slight abuse of notation) $L \Phi: L G \times M \longrightarrow L M$ denote the map $(\gamma, x) \longmapsto \gamma_{x}$, with $\gamma_{x}(t)=\Phi_{\gamma(t)}(x)$. Consider the hermitean line bundle

$$
K:=F^{*} \mathrm{~s}^{*} L \Phi^{*} \mathcal{T} \mathcal{G} \otimes\left(1_{P_{0} G} \times \Phi_{\mathrm{ev}_{1}}\right)^{*} J_{1} \otimes J_{0}
$$

on the diffeological space

$$
Y_{f_{1}, f_{0}}:=\left(\left(f_{1} f_{0}\right)^{*} P_{0} G \times_{c} f_{1}^{*} P_{0} G \times_{c} f_{0}^{*} P_{0} G\right) \times M .
$$


We claim that the bundle $K$ descends along the projection $p_{1}: Y_{f_{1}, f_{0}} \longrightarrow\left(f_{1} f_{0}\right)^{*} P_{0} G \times$ $M$. The descended bundle is the hermitean line bundle underlying the product $\left(f_{1}, J_{1}, J_{1}\right) \otimes$ $\left(f_{0}, J_{0}, J_{0}\right)$.

We thus endow the bundle $K$ with an isomorphism $\kappa:\left(p_{1}\right)_{0}^{*} K \longrightarrow\left(p_{1}\right)_{1}^{*} K$ over $Y_{f_{1}, f_{0}}^{[2]}$, which is required to satisfy a cocycle relation over $Y_{f_{1}, f_{0}}^{[3]}$. An element of $Y_{f_{1}, f_{0}}^{[2]}$ can be identified with a pair of triples $\left(\left(\gamma_{10}, \gamma_{1}, \gamma_{0}\right),\left(\gamma_{10}, \gamma_{1}^{\prime}, \gamma_{0}^{\prime}\right)\right)$, where $\left(\gamma_{i}, \gamma_{i}^{\prime}\right) \in\left(P_{0} G\right)^{[2]}$ for $i=0,1$. We define the isomorphism $\kappa$ as the composition of the fusion product $\lambda$ on $\mathcal{T G}$ with $\left(1 \times \Phi_{\mathrm{ev}_{1}}\right)^{*} J_{1} \otimes J_{0}$. Then the cocycle condition simply follows from the compatibility condition (5.34) and the associativity of the fusion product. (We also need to use thin reparameterisations, but these are implemented in a completely coherent way by the thin-homotopy invariant connection on $\mathcal{T} \mathcal{G}$.)

Thus we obtain a descended hermitean line bundle $\mathfrak{D e s}(K, \kappa)$ on $\left(f_{1} f_{0}\right)^{*} P_{0} G \times M$ (for descent properties of diffeological vector bundles, see [Bun20a]). Applying the fusion product in the first tensor factor of $K$, we obtain an isomorphism which (by the associativity of $\lambda$ ) descends to an isomorphism

$$
J_{K}: d_{1}^{*} \mathfrak{D} \mathfrak{e s}(K, \kappa) \longrightarrow\left({\widehat{f_{1} f_{0}}}^{[2]} \times 1\right)^{*} \mathrm{~L}^{\dashv} \otimes d_{0}^{*} \mathfrak{D e s}(K, \kappa)
$$

over $\left(\left(f_{1} f_{0}\right)^{*} P_{0} G\right)^{[2]} \times M$. Again by the associativity of $\lambda$ and thin-homotopy invariance, the pair $\left(\mathfrak{D e s}(K, \kappa), J_{K}\right)$ satisfies the relation (5.34), and hence it makes sense to set

$$
\left(f_{1}, J_{1}, J_{1}\right) \otimes\left(f_{0}, J_{0}, J_{0}\right):=\left(f_{1} f_{0}, \mathfrak{D e s}(K, \kappa), J_{K}\right) .
$$

The action of the product $\otimes$ in $\mathfrak{D e s L}$ on morphisms simply sends $\left(\psi_{1}, \psi_{0}\right)$ to the descent along $p_{1}$ of the isomorphism $1_{\mathcal{T} \mathcal{G}} \otimes\left(1 \times \Phi_{\mathrm{ev}_{1}}\right)^{*} \psi_{1} \otimes \psi_{0}$. The unitors of $\otimes$ are readily obtained from the construction, and the associator is defined from the fusion product; its coherence is yet another application of the associativity of $\lambda$ and the superficiality of the parallel transport on $\mathcal{T} \mathcal{G}$. Inverses are constructed analogously to (5.26). Finally, all constructions are compatible with pullbacks along maps $\varphi: c^{\prime} \longrightarrow c$ of Cartesian spaces, so that we obtain the structure of a smooth 2-group on $\mathfrak{D e s}$.

Theorem 5.36. There is a weakly commutative diagram of smooth 2-groups

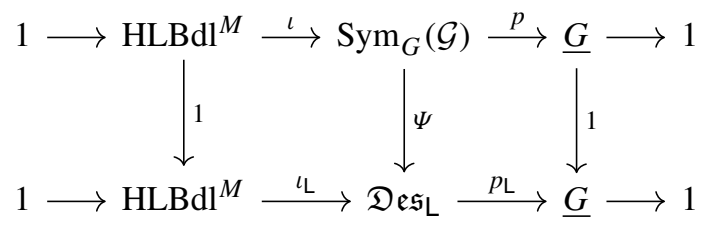

where the functor $\Psi$ is an equivalence.

Proof. By the functoriality of $\mathcal{G} \longmapsto \operatorname{Sym}_{G}(\mathcal{G})$ (see Sect. 5.4) we can assume that we are in the case where $\mathcal{G}^{\prime}=\mathcal{R} \mathcal{T}(\mathcal{G})$ is the regression of a transgression, so that we have direct access to our construction of a parallel transport on $\mathcal{G}^{\prime}$ from Sect. 4.4. We start by constructing the functor $\Psi$. For this, we construct a diagram in the 2-category $\mathcal{H}$ of the form

$$
\operatorname{Sym}_{G}\left(\mathcal{G}^{\prime}\right) \stackrel{\varpi^{*}}{\longrightarrow} \mathfrak{D e s}\left(\operatorname{Sym}_{G}\left(\mathcal{G}^{\prime}\right)\right) \frac{\operatorname{Hom}_{1}\left(\mathrm{pt}_{1}^{\mathcal{G}^{\prime}}, \cdot\right)}{(\cdot) \otimes \mathrm{pt}_{1}^{\mathcal{G}^{\prime}}} \mathfrak{D e s L}
$$


and the functor $\Psi$ is the composition from left to right. Each of the functors in (5.38) is an equivalence of categories fibred in groupoids over Cart, and hence so is $\Psi$.

For a smooth map $f: c \longrightarrow G$, let $\varpi_{f}: f^{*} P_{0} G \times M \longrightarrow c \times M$ denote the canonical projection. First we define the category $\mathfrak{D} \mathfrak{e s}\left(\operatorname{Sym}_{G}\left(\mathcal{G}^{\prime}\right)\right)$. It is obtained via the Grothendieck construction applied to the presheaf $\mathfrak{D e s}^{\mathrm{PSh}}\left(\operatorname{Sym}_{G}\left(\mathcal{G}^{\prime}\right)\right)$ of groupoids on $\underline{G}$, which assigns to a smooth map $f: c \longrightarrow G$ the groupoid $\mathfrak{D e s}^{\mathrm{PSh}}\left(\operatorname{Sym}_{G}\left(\mathcal{G}^{\prime}\right)\right)(f)$ where

- objects are pairs $(A, \alpha)$ of a 1-isomorphism $A$ : $\varpi_{f}^{*} \mathcal{G}^{\prime} \longrightarrow \varpi_{f}^{*} \Phi_{f}^{*} \mathcal{G}^{\prime}$ over $f^{*} P_{0} G \times$ $M$ and a 2-isomorphism $\alpha: d_{1}^{*} A \longrightarrow d_{0}^{*} A$ over $\left(f^{*} P_{0} G\right)^{[2]} \times M$, which is coherent over $\left(f^{*} P_{0} G\right)^{[3]} \times M$, and

- morphisms $(A, \alpha) \longrightarrow\left(A^{\prime}, \alpha^{\prime}\right)$ are given by 2-isomorphisms $\psi: A \longrightarrow A^{\prime}$ satisfying $\alpha^{\prime} \circ d_{1}^{*} \psi=d_{0}^{*} \psi \circ \alpha$.

The functor $\varpi^{*}$ simply pulls back 1-morphisms $A: \operatorname{pr}_{M}^{*} \mathcal{G}^{\prime} \longrightarrow \Phi_{f}^{*} \mathcal{G}^{\prime}$ along the subductions $\varpi_{f}$. This functor is an equivalence since morphisms of bundle gerbes satisfy descent ${ }^{3}$ [Bun17, Theorem A.19].

Next we introduce some notation: we define the map

$$
\begin{aligned}
P_{0} \Phi: P_{0} G \times M & \longrightarrow P M \\
(\gamma, x) & \longmapsto \gamma_{x},
\end{aligned}
$$

where

$$
\gamma_{x}(t)=\Phi_{\gamma(t)}(x)=: P_{0} \Phi(\gamma, x)(t)
$$

for all $t \in[0,1]$. Observe that

$$
\mathrm{ev}_{0} \circ P_{0} \Phi=\operatorname{pr}_{M} \quad \text { and } \quad \operatorname{ev}_{1} \circ P_{0} \Phi=\Phi \circ\left(\mathrm{ev}_{1} \times 1_{M}\right) .
$$

Thus the pullback of the parallel transport 1-isomorphism (4.9) by the map $P_{0} \Phi$ is a morphism

$$
\left(P_{0} \Phi\right)^{*} \mathrm{pt}_{1}^{\mathcal{G}^{\prime}}: \mathrm{pr}_{M}^{*} \mathcal{G}^{\prime} \longrightarrow\left(\mathrm{ev}_{1} \times 1_{M}\right)^{*} \Phi^{*} \mathcal{G}^{\prime}
$$

in $\operatorname{BGrb}\left(P_{0} G \times M\right)$. Given a smooth map $f: c \longrightarrow G$, we obtain a smooth map

$$
P_{0} \Phi \circ\left(\widehat{f} \times 1_{M}\right): f^{*} P_{0} G \times M \longrightarrow P M .
$$

It satisfies

$$
\mathrm{ev}_{0} \circ P_{0} \Phi \circ\left(\widehat{f} \times 1_{M}\right)=\operatorname{pr}_{M} \quad \text { and } \quad \operatorname{ev}_{1} \circ P_{0} \Phi \circ\left(\widehat{f} \times 1_{M}\right)=\Phi_{f} \circ \varpi_{f},
$$

where $\varpi_{f}: f^{*} P_{0} G \times M \longrightarrow c \times M$ is the projection. Hence we obtain a morphism

$$
\operatorname{pt}_{f}^{\mathcal{G}^{\prime}}:=\left(P_{0} \Phi \circ(\widehat{f} \times 1)\right)^{*} \operatorname{pt}_{1}^{\mathcal{G}^{\prime}}: \varpi_{f}^{*} \operatorname{pr}_{M}^{*} \mathcal{G}^{\prime} \longrightarrow \varpi_{f}^{*} \Phi_{f}^{*} \mathcal{G}^{\prime},
$$

which is defined over $f^{*} P_{0} G \times M$. By Proposition 4.18 there is a canonical 2-isomorphism

$$
\left(d_{0}^{*} \mathrm{pt}{\stackrel{\mathcal{G}^{\prime}}{f}}^{-1} \circ d_{1}^{*} \mathrm{pt} \mathrm{\mathcal {G }}_{f} \stackrel{\cong}{\longrightarrow}\left(L \Phi \circ\left(\tau \times 1_{M}\right) \circ\left(\widehat{f}^{[2]} \times 1_{M}\right)\right)^{*} \mathcal{T} \mathcal{G} \cong\left(\widehat{f}^{[2]} \times 1_{M}\right)^{*} \mathrm{~L}^{\dashv \vee}\right.
$$

\footnotetext{
3 In [Bun17] the descent property was proven along surjective submersions of manifolds, but the proof directly carries over to subductions of diffeological spaces.
} 
of 1-automorphisms of the pullback of $\operatorname{pr}_{M}^{*} \mathcal{G}^{\prime}$ to $\left(f^{*} P_{0} G\right)^{[2]} \times M$, where $\tau\left(\gamma_{0}, \gamma_{1}\right)=$ $\left(\gamma_{1}, \gamma_{0}\right)$. Equivalently, this is a 2-isomorphism

$$
\beta_{f}: d_{0}^{*} \mathrm{pt}{\mathcal{\mathcal { G } ^ { \prime }}}_{f}^{\cong}\left(\widehat{f}^{[2]} \times 1_{M}\right)^{*} \mathrm{~L}^{\dashv} \otimes d_{1}^{*} \mathrm{pt}{ }_{f}^{\mathcal{G}^{\prime}}
$$

of 1-isomorphisms $\varpi_{f}^{*} \operatorname{pr}_{M}^{*} \mathcal{G}^{\prime} \longrightarrow \varpi_{f}^{*} \Phi_{f}^{*} \mathcal{G}^{\prime}$ over $\left(f^{*} P_{0} G\right)^{[2]} \times M$.

Now we come to the definition of the functor $(\cdot) \otimes p t_{1}^{\mathcal{G}^{\prime}}$. Given an object $(f, J, J) \in$ $\mathfrak{D e s}$, define a morphism of bundle gerbes over $f^{*} P_{0} G \times M$ via

$$
J \otimes \mathrm{pt}_{f}^{\mathcal{G}^{\prime}}: \varpi_{f}^{*} \mathrm{pr}_{M}^{*} \mathcal{G}^{\prime} \longrightarrow \varpi_{f}^{*} \Phi_{f}^{*} \mathcal{G}^{\prime}
$$

Using the 2-isomorphism (5.39), we obtain a 2-isomorphism

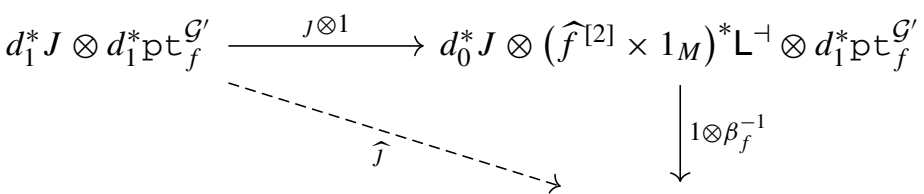

$$
\begin{aligned}
& d_{0}^{*} J \otimes d_{0}^{*} \mathrm{p} t_{f}^{\mathcal{G}^{\prime}}
\end{aligned}
$$

over $\left(f^{*} P_{0} G\right)^{[2]} \times M$. By construction, the 2-isomorphism $\widehat{J}$ is coherent over $\left(f^{*} P_{0} G\right)^{[3]}$ $\times M$, and thus the pair $\left(J \otimes \mathrm{pt}_{f}^{\mathcal{G}^{\prime}}, \widehat{J}\right)$ defines a descent datum (with respect to the subduction $\left.f^{*} P_{0} G \times M \longrightarrow c \times M\right)$ for a 1-isomorphism of bundle gerbes $\operatorname{pr}_{M}^{*} \mathcal{G}^{\prime} \longrightarrow \Phi_{f}^{*} \mathcal{G}^{\prime}$. Analogously, morphisms in $\mathfrak{D} \mathfrak{e} \mathfrak{L}$ give rise to morphisms of descent data as constructed above. This defines the functor

$$
(\cdot) \otimes \mathrm{pt}_{1}^{\mathcal{G}^{\prime}}: \mathfrak{D e s L} \longrightarrow \mathfrak{D e s}\left(\operatorname{Sym}_{G}\left(\mathcal{G}^{\prime}\right)\right) .
$$

This is a functor of categories fibred in groupoids over Cart by the compatibility of pullbacks of bundles and their morphisms with the tensor product.

Finally, we introduce an inverse functor $\operatorname{Hom}_{1}\left(\mathrm{pt}_{1}^{\mathcal{G}^{\prime}}, \cdot\right)$ for $(\cdot) \otimes \mathrm{p} t_{1}^{\mathcal{G}^{\prime}}$. An object $(f, A, \alpha) \in \mathfrak{D e s}\left(\operatorname{Sym}_{G}\left(\mathcal{G}^{\prime}\right)\right)$ consists, in particular, of a 1-isomorphism $A: \varpi_{f}^{*} \operatorname{pr}_{M}^{*} \mathcal{G}^{\prime} \longrightarrow \varpi_{\mathcal{G}^{\prime}}^{*} \Phi_{f}^{*} \mathcal{G}^{\prime}$ of bundle gerbes over $f^{*} P_{0} G \times M$. Another such morphism is given by $\mathrm{pt}_{f}^{\mathcal{G}^{\prime}}$. We can hence use the internal hom-functor $\mathrm{Hom}_{1}$ in the 2category $\operatorname{BGrb}\left(f^{*} P_{0} G \times M\right)$ (see [Bun17, Section 3.2] and also [BW18, Section 2.1]) to produce a hermitean line bundle

$$
\operatorname{Hom}_{1}\left(\mathrm{pt}_{f}^{\mathcal{G}^{\prime}}, A\right) \in \operatorname{HLBdl}\left(f^{*} P_{0} G \times M\right)
$$

This comes with an isomorphism over $\left(f^{*} P_{0} G\right)^{[2]} \times M$ defined by the diagram

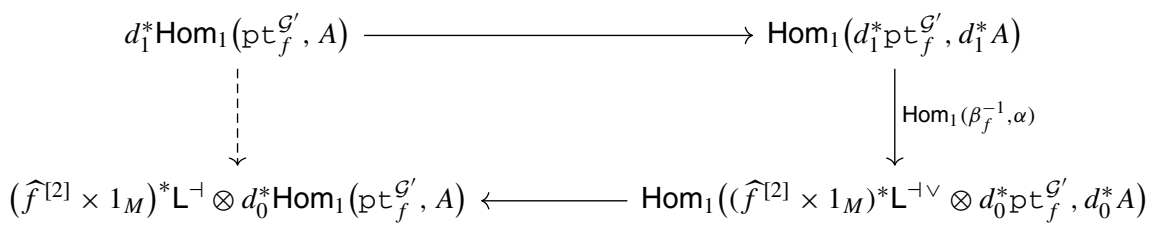


where the 2-isomorphism $\beta_{f}$ stems from (5.39) and where the lower horizontal isomorphism of line bundles stems from the (categorified) linearity of Hom 1 [Bun17, Theorem 3.63]. It follows from the properties of $\alpha$ and $\beta_{f}$ that $\operatorname{Hom}_{1}\left(\mathrm{pt}_{f}^{\mathcal{G}^{\prime}}, A\right)$ defines an object in $(\mathfrak{D e s})_{\mid f}$. By mapping a morphism $\psi$ in $\mathfrak{D e s}\left(\operatorname{Sym}_{G}\left(\mathcal{G}^{\prime}\right)\right)_{\mid f}$ to $\operatorname{Hom}_{1}\left(\operatorname{pt}_{f}^{\mathcal{G}^{\prime}}, \psi\right)$, we obtain a functor

$$
\operatorname{Hom}_{1}\left(\mathrm{pt}_{1}^{\mathcal{G}^{\prime}}, \cdot\right): \mathfrak{D e s}\left(\operatorname{Sym}_{G}\left(\mathcal{G}^{\prime}\right)\right) \longrightarrow \mathfrak{D e s L}
$$

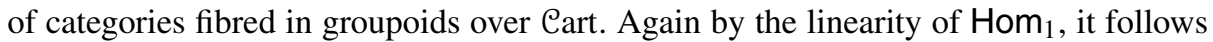
straightforwardly that $(\cdot) \otimes \mathrm{pt}_{1}^{\mathcal{G}^{\prime}}$ and $\operatorname{Hom}_{1}\left(\mathrm{pt}_{1}^{\mathcal{G}^{\prime}}, \cdot\right)$ are mutually inverse functors.

To conclude the proof, we need to check that $\Psi$ is compatible with the monoid structures on $\operatorname{Sym}_{G}\left(\mathcal{G}^{\prime}\right)$ and on $\mathfrak{D e s}_{\mathrm{L}}$. For $i=0,1$, let $f_{i}: c \longrightarrow G$ be smooth maps from $c \in$ Cart to $G$, and consider objects $A_{i} \in \operatorname{Sym}_{G}\left(\mathcal{G}^{\prime}\right)_{\mid f_{i}}$ in the fibres over $f_{i}$. By the explicit construction of the 2-group structure on $\mathfrak{D} \mathfrak{e} \mathfrak{S}_{\mathrm{L}}$ in the proof of Proposition 5.35 it follows that the hermitean line bundle underlying the object $\Psi\left(f_{1}, A_{1}\right) \otimes \Psi\left(f_{0}, A_{0}\right)$ of $\mathfrak{D e s} L$ is given as the descent of the bundle

$$
\begin{aligned}
\mathrm{L}^{\dashv} & \otimes\left(1 \times \Phi_{\mathrm{ev}_{1}}\right)^{*}\left(\operatorname{Hom}_{1}\left(\mathrm{pt}_{f_{1}}^{\mathcal{G}^{\prime}}, \varpi_{f_{1}}^{*} A_{1}\right)\right) \otimes \operatorname{Hom}_{1}\left(\mathrm{pt}_{f_{0}}^{\mathcal{G}^{\prime}}, \varpi_{f_{0}}^{*} A_{0}\right) \\
& \cong \mathrm{L}^{\dashv} \otimes \operatorname{Hom}_{1}\left(\left(1 \times \Phi_{\mathrm{ev}_{1}}\right)^{*} \widehat{f}_{1}^{*} \mathrm{pt}_{1}^{\mathcal{G}^{\prime}},\left(1 \times \Phi_{\mathrm{ev}_{1}}\right)^{*} \varpi_{f_{1}}^{*} A_{1}\right) \otimes \operatorname{Hom}_{1}\left(\widehat{f}_{0}^{*} \mathrm{pt}_{1}^{\mathcal{G}^{\prime}}, \varpi_{f_{0}}^{*} A_{0}\right) \\
& \cong \mathrm{L}^{\dashv} \otimes \operatorname{Hom}_{1}\left(\left(1 \times \Phi_{\mathrm{ev}_{1}}\right)^{*} \widehat{f}_{1}^{*} \mathrm{pt}_{1}^{\mathcal{G}^{\prime}} \circ \widehat{f}_{0}^{*} \mathrm{pt}_{1}^{\mathcal{G}^{\prime}},\left(1 \times \Phi_{\mathrm{ev}_{1}}\right)^{*} \varpi_{f_{1}}^{*} A_{1} \circ \varpi_{f_{0}}^{*} A_{0}\right) \\
& \cong \operatorname{Hom}_{1}\left(\left(\widehat{f_{1} f_{0}}\right)^{*} \mathrm{pt}_{1}^{\mathcal{G}^{\prime}}, \varpi_{f_{1} f_{0}}^{*}\left(\Phi_{f_{0}}^{*} A_{1} \circ A_{0}\right)\right) .
\end{aligned}
$$

The first and second isomorphisms follow from the properties of the internal hom-functor $\mathrm{Hom}_{1}$. The third isomorphism is a direct application of Proposition 4.18. The bundle in the last line is the line bundle underlying the object $\Psi\left(\left(f_{1}, A_{1}\right) \otimes\left(f_{0}, A_{0}\right)\right)$ of $\mathfrak{D e S L}$ (see (5.25)). Hence the canonical isomorphism (5.40) establishes the compatibility of $\Psi$. Its coherence again follows from the properties of the transgression line bundle $\mathcal{T} \mathcal{G}$. The proofs that $\Psi$ respects unitors as well as the weak commutativity of the diagram (5.37) are straightforward.

5.6. Equivariant bundle gerbes. We shall now investigate the relation between sections of the smooth 2-group extension $\operatorname{Sym}_{G}(\mathcal{G}) \longrightarrow \underline{G}$ and equivariant structures on $\mathcal{G}$. We first recall an explicit definition of an equivariant bundle gerbe from [GSW11] (see also [M+17]), which can be understood very nicely from the perspective of the formalism developed in [NS11].

Let $G$ be a connected Lie group, $M$ a manifold with $G$-action $\Phi: G \times M \longrightarrow M$, and $\mathcal{G}$ a hermitean bundle gerbe over $M$. Corresponding to the action groupoid $G \times M \rightrightarrows M$ there is a simplicial manifold

$$
\cdots G^{\times 2} \times M \rightleftharpoons G \times M \rightleftharpoons M,
$$

with face maps $d_{i}: G^{\times n} \times M \longrightarrow G^{\times n-1} \times M$ for $0 \leq i \leq n$ given by

$$
d_{i}\left(g_{0}, g_{1}, \ldots, g_{n-1}, x\right)=\left\{\begin{array}{ll}
\left(g_{0}, g_{1}, \ldots, g_{n-2}, \Phi_{g_{n-1}}(x)\right) & \text { for } i=n \\
\left(g_{0}, g_{1}, \ldots, g_{i-1} g_{i}, \ldots, g_{n-1}, x\right) & \text { for } 0<i<n \\
\left(g_{1}, \ldots, g_{n-1}, x\right) & \text { for } i=0
\end{array} .\right.
$$


On $G \times M$, the face maps $d_{0}=\operatorname{pr}_{M}$ and $d_{1}=\Phi$ are the source and target maps of the action groupoid.

Definition 5.41. Let $G$ be a connected Lie group, $M$ a manifold with $G$-action $\Phi: G \times$ $M \longrightarrow M$, and $\mathcal{G}$ a hermitean bundle gerbe over $M$. A $G$-equivariant structure on $\mathcal{G}$ consists of a 1-isomorphism $A: \operatorname{pr}_{M}^{*} \mathcal{G} \longrightarrow \Phi^{*} \mathcal{G}$ over $G \times M$ and a 2-isomorphism $\chi: d_{2}^{*} A \circ d_{0}^{*} A \longrightarrow d_{1}^{*} A$ over $G^{\times 2} \times M$ such that

$$
d_{2}^{*} \chi \circ\left(1_{\left(d_{3} \circ d_{2}\right)^{*} A} \circ d_{0}^{*} \chi\right)=d_{1}^{*} \chi \circ\left(1_{\left(d_{0} \circ d_{0}\right)^{*} A} \circ d_{3}^{*} \chi\right)
$$

over $G^{\times 3} \times M$. A morphism $(A, \chi) \longrightarrow\left(A^{\prime}, \chi^{\prime}\right)$ between equivariant structures on $\mathcal{G}$ consists of a 2-isomorphism $\vartheta: A \longrightarrow A^{\prime}$ such that the diagram

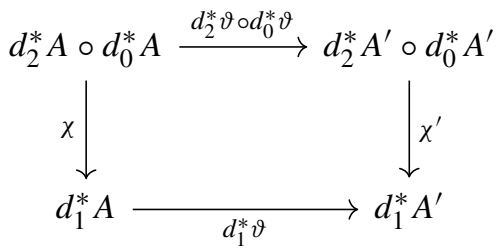

commutes. We denote by $\mathcal{E}(\mathcal{G})$ the groupoid of equivariant structures on $\mathcal{G}$.

A splitting of $p: \operatorname{Sym}_{G}(\mathcal{G}) \longrightarrow G$ is a smooth 2-group homomorphism $s: \underline{G} \longrightarrow$ $\operatorname{Sym}_{G}(\mathcal{G})$ such that $p \circ s=1_{G}$. We assume here for simplicity and without loss of generality that unitors are strictly preserved. We denote by $\mathcal{S}\left(\underline{G} ; \operatorname{Sym}_{G}(\mathcal{G})\right)$ the groupoid of splittings of $p: \operatorname{Sym}_{G}(\mathcal{G}) \longrightarrow \underline{G}$. Concretely, a splitting consists of

- a 1-isomorphism $s(f): \operatorname{pr}_{M}^{*} \mathcal{G} \longrightarrow \Phi_{f}^{*} \mathcal{G}$ of bundle gerbes on $c \times M$ for every Cartesian space $c \in$ Cart and sections $f \in G(c)$,

- a 2-isomorphism $s(\varphi): s(f) \longrightarrow \varphi^{*} s\left(f^{\prime}\right)$ for every morphism $\varphi: f \longrightarrow f^{\prime}$ in $\underline{G}$ , and

- a 2-isomorphism $s(f) \otimes s\left(f^{\prime}\right) \longrightarrow s\left(f f^{\prime}\right) \operatorname{in}_{\operatorname{Sym}_{G}}(\mathcal{G})$ for every $f, f^{\prime} \in G(c)$, such that $\varphi^{*} s\left(\varphi^{\prime}\right) \circ s(\varphi)=s\left(\varphi^{\prime} \circ \varphi\right)$ and the diagram

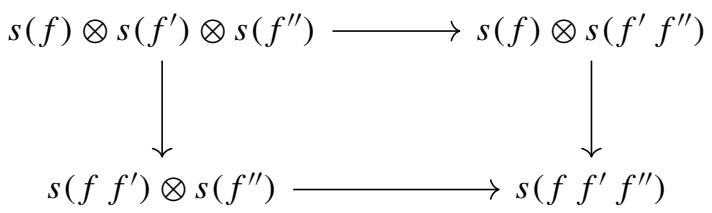

commutes. A morphism $\omega: s \longrightarrow s^{\prime}$ of splittings consists of 2-isomorphisms $\omega(f)$ : $s(f) \longrightarrow s^{\prime}(f)$ in $\operatorname{BGrb}(c \times M)$ for all $f \in G(c)$ such that the diagrams
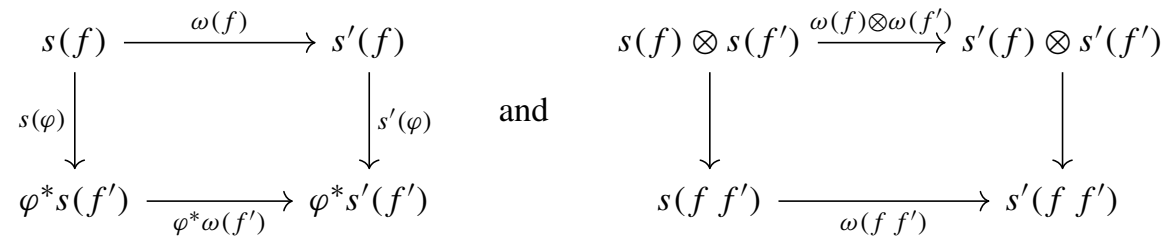

commute. 
In what follows we construct an equivalence

$$
\Xi: \mathcal{E}(\mathcal{G}) \longrightarrow \mathcal{S}\left(\underline{G} ; \operatorname{Sym}_{G}(\mathcal{G})\right)
$$

of categories. Let $(A, \chi)$ be an equivariant structure on $\mathcal{G}$ and $(f: c \longrightarrow G) \in G(c)$. Pulling back $A$ along $\Phi_{f}$ gives rise to a 1-isomorphism $\Phi_{f}^{*} A: \operatorname{pr}_{M}^{*} \mathcal{G} \longrightarrow \Phi_{f}^{*} \mathcal{G}$ over $c \times M$. We can define the section $\Xi(A, \chi): \underline{G} \longrightarrow \operatorname{Sym}_{G}(\mathcal{G})$ by $\Xi(A, \chi)(f)=$ $\Phi_{f}^{*} A: \operatorname{pr}_{M}^{*} \mathcal{G} \longrightarrow \Phi_{f}^{*} \mathcal{G}$. The 2-isomorphisms $\Xi(A, \chi)(\varphi)$ are induced by general properties of pullbacks and the 2-isomorphism $\chi$ induces the 2-isomorphism encoding the compatibility with multiplication. The action of $\Xi$ on morphisms of equivariant structures is again by pullback along $\Phi_{f}$.

Theorem 5.42. The functor $\Xi: \mathcal{E}(\mathcal{G}) \longrightarrow \mathcal{S}\left(\underline{G} ; \operatorname{Sym}_{G}(\mathcal{G})\right)$ is an equivalence of categories.

Proof. We start by showing that $\Xi$ is essentially surjective. Let $s: \underline{G} \longrightarrow \operatorname{Sym}_{G}(\mathcal{G})$ be a splitting. We pick a good open cover $\left\{c_{i}\right\}_{i \in \Lambda}$ of $G$. This induces good open covers of $G^{\times 2}$ and $G^{\times 3}$ given by $\left\{c_{i} \times c_{j}\right\}_{i, j \in \Lambda}$ and $\left\{c_{i} \times c_{j} \times c_{k}\right\}_{i, j, k \in \Lambda}$, respectively. Every $c_{i}$ comes with an embedding $f_{i}: c_{i} \longrightarrow G$ and hence can be regarded as an object of $\underline{G}$. Applying the section $s: \underline{G} \longrightarrow \operatorname{Sym}_{G}(\mathcal{G})$ to all elements of the open cover provides a collection of compatible 1-isomorphisms

$$
s\left(c_{i}\right):=s\left(f_{i}: c_{i} \longrightarrow G\right): \operatorname{pr}_{M}^{*} \mathcal{G} \longrightarrow \Phi_{f_{i}}^{*} \mathcal{G} .
$$

On double intersections $c_{i j}:=c_{i} \cap c_{j}$ we get coherent 2-isomorphisms ${ }^{4}$

$$
s\left(c_{i j}\right): s\left(c_{i}\right)_{\mid c_{i j}} \longrightarrow s\left(f_{i \mid c_{i j}}\right)=s\left(f_{j \mid c_{i j}}\right) \longrightarrow s\left(c_{j}\right)_{\mid c_{i j}},
$$

since $\operatorname{Sym}_{G}(\mathcal{G}) \longrightarrow$ Cart is a Grothendieck fibration. Hence the 1-isomorphisms $s\left(c_{i}\right)$ glue together to a 1-isomorphism $A_{s}: \operatorname{pr}_{M}^{*} \mathcal{G} \longrightarrow \Phi^{*} \mathcal{G}$ over $G \times M$.

Let $\mathrm{pr}_{1}, \mathrm{pr}_{2}: G^{\times 2} \longrightarrow G$ be the projections to the first and second factors, and $m: G^{\times 2} \longrightarrow G$ the multiplication in $G$. From $A_{s}$ we can construct three 1-morphisms $\operatorname{pr}_{1}^{*} A_{s}, \operatorname{pr}_{2}^{*} A_{s}$, and $m^{*} A_{s}$ over $G^{\times 2} \times M$. We would like to show that these 1-morphisms are canonically isomorphic to the 1-morphisms constructed from the good open cover $\left\{c_{i} \times c_{j}\right\}_{i, j \in \Lambda}$ by applying $s$ to the morphisms $\operatorname{pr}_{1 \mid c_{i} \times c_{j}}, \operatorname{pr}_{2 \mid c_{i} \times c_{j}}$, and $m_{\mid c_{i} \times c_{j}}$ on $c_{i} \times c_{j} \longrightarrow G$, respectively. For this, consider the commutative diagram

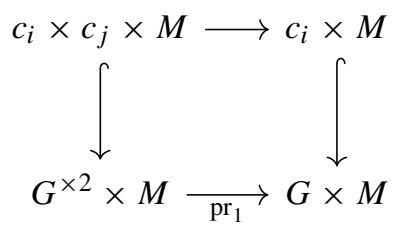

which implies that

$$
\operatorname{pr}_{1 \mid c_{i} \times c_{j}}^{*} A_{s}=\operatorname{pr}_{1 \mid c_{i} \times c_{j}}^{*} s\left(c_{i} \hookrightarrow G\right) \stackrel{\cong}{\longrightarrow} s\left(c_{i} \times c_{j} \stackrel{\mathrm{pr}_{1}}{\longrightarrow} c_{i} \hookrightarrow G\right),
$$

\footnotetext{
${ }^{4}$ Here we interpret $s$ as a map of stacks, i.e. a natural transformation of 2-functors Cart $\longrightarrow$ Cat, via the inverse Grothendieck construction.
} 
where the 2-isomorphism is part of the data of the section $s$. The same argument shows the claim for $\mathrm{pr}_{2}$. To show the corresponding statement for $m$ we need to pick a refinement $\left\{\widetilde{c}_{a}\right\}_{a \in \tilde{\Lambda}}$ of the cover $\left\{c_{i} \times c_{j}\right\}_{i, j \in \Lambda}$ such that the diagram

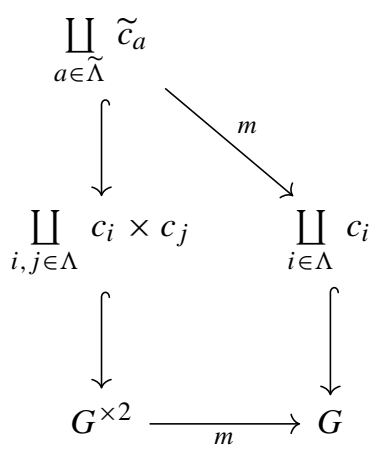

commutes. The cover $\left\{\widetilde{c}_{a}\right\}_{a \in \tilde{\Lambda}}$ can be constructed by choosing a common refinement of the covers $\left\{c_{i} \times c_{j}\right\}_{i, j \in \Lambda}$ and $\left\{m^{-1}\left(c_{i}\right)\right\}_{i \in \Lambda}$ of $G^{\times 2}$.

The multiplication of $c_{i} \times c_{j} \stackrel{\mathrm{pr}_{1}}{\longrightarrow} G$ with $c_{i} \times c_{j} \stackrel{\mathrm{pr}_{2}}{\longrightarrow} G$ in $\underline{G}$ is $c_{i} \times c_{j} \stackrel{m}{\longrightarrow} G$. The structure of a smooth 2-group homomorphism on $s$ now provides natural 2-isomorphisms

$$
s\left(c_{i} \times c_{j} \stackrel{\mathrm{pr}_{1}}{\longrightarrow} G\right) \otimes s\left(c_{i} \times c_{j} \stackrel{\mathrm{pr}_{2}}{\longrightarrow} G\right) \longrightarrow s\left(c_{i} \times c_{j} \stackrel{m}{\longrightarrow} G\right)
$$

which glue together to a 2-isomorphism $\chi_{s}: \Phi^{*}\left(\mathrm{pr}_{1}^{*} A_{S}\right) \circ\left(\mathrm{pr}_{2}^{*} A_{s}\right) \longrightarrow m^{*} A_{s}$ over $G^{\times 2} \times M$ because (5.43) is a 2-isomorphism of smooth 1-isomorphisms. The coherence condition for $\chi_{s}$ over $G^{\times 3} \times M$ follows from the observation that the various pullbacks to $G^{\times 3} \times M$ can be constructed by applying $s$ to different functions from $c_{i} \times c_{j} \times c_{k}$ to $G$ and the coherence condition for $s$. This shows that $\Xi$ is essentially surjective.

We next show that the functor $\Xi$ is faithful: let $\vartheta, \vartheta^{\prime}:(A, \chi) \longrightarrow\left(A^{\prime}, \chi^{\prime}\right)$ be isomorphisms of equivariant structures on $\mathcal{G}$ such that $\Xi\left(\vartheta^{\prime}\right)=\Xi(\vartheta)$, and let $g \in G$. We can take $c=\mathbb{R}^{0}$ and $f: c \longrightarrow G$ to be the constant map at $g$ to conclude that $\varphi_{\mid\{g\} \times M}=\Xi(A, \chi)(\varphi)(f)$ and $\varphi_{\mid\{g\} \times M}=\Xi\left(A^{\prime}, \chi^{\prime}\right)(\varphi)(f)$ agree. Hence the two isomorphisms agree pointwise and the statement follows.

Finally, we show that the functor $\Xi$ is full: let $(A, \chi)$ and $\left(A^{\prime}, \chi^{\prime}\right)$ be equivariant structures on $\mathcal{G}$ and $\omega: \Xi(A, \chi) \longrightarrow \Xi\left(A^{\prime}, \chi^{\prime}\right)$ a morphism of splittings. Evaluating $\omega$ on the good open cover $\left\{c_{i}\right\}_{i \in \Lambda}$ from above provides isomorphisms $\omega: A_{\mid c_{i} \times M} \longrightarrow$ $A_{\mid c_{i} \times M}^{\prime}$. Since $\omega$ is a morphism of splittings, these morphisms glue together to a 2 isomorphism $\vartheta_{\omega}: A \longrightarrow A^{\prime}$. That this is an isomorphism of equivariant structures follows from the coherence conditions for $\omega$ and the observation that it suffices to check the conditions locally.

Corollary 5.44. A bundle gerbe $\mathcal{G}$ on a manifold $M$ admits an equivariant structure if and only if the 2-group extension

$$
1 \longrightarrow \operatorname{HLBdl}^{M} \longrightarrow \operatorname{Sym}_{G}(\mathcal{G}) \longrightarrow \underline{G} \longrightarrow 1
$$

admits a splitting. 
Definition 5.45. Let $(\mathcal{G}, A, \chi)$ and $\left(\mathcal{G}^{\prime}, A^{\prime}, \chi^{\prime}\right)$ be $G$-equivariant bundle gerbes on $M$. An equivariant 1-isomorphism $\mathcal{G} \longrightarrow \mathcal{G}^{\prime}$ consists of a 1-morphism of bundle gerbes $E: \mathcal{G} \longrightarrow \mathcal{G}^{\prime}$ together with a 2-morphism $\gamma: \Phi^{*} E \longrightarrow \operatorname{pr}_{M}^{*} E$ defined by the diagram

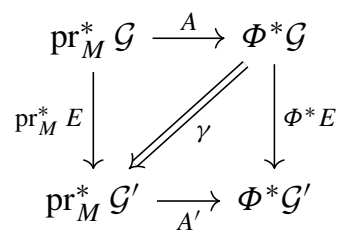

in $\operatorname{BGrb}(G \times M)$, such that for every $g, g^{\prime} \in G$ there is an equality of diagrams
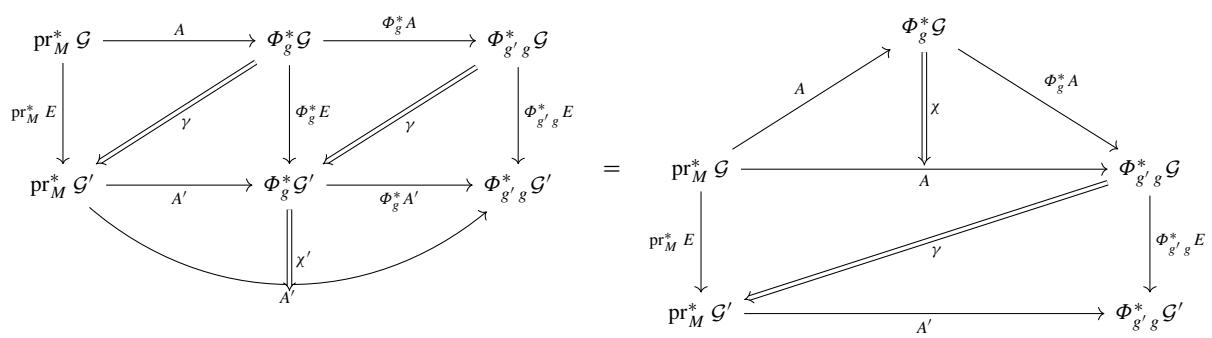

Being an equivariant 1-isomorphism is a structure and not a property: given a 1morphism $E: \mathcal{G} \longrightarrow \mathcal{G}^{\prime}$ of bundle gerbes there is a set $\mathcal{E}(E)$ of equivariant structures on $E$. According to Theorem 5.42 we can describe the equivariant structures on $\mathcal{G}$ and $\mathcal{G}^{\prime}$ by splittings $s: \underline{G} \longrightarrow \operatorname{Sym}_{G}(\mathcal{G})$ and $s^{\prime}: \underline{G} \longrightarrow \operatorname{Sym}_{G}\left(\mathcal{G}^{\prime}\right)$. We shall now give a description of an equivariant structure on $E$ using these homomorphisms of 2-groups. For this, recall from the end of Sect. 5.4 that any 1-isomorphism $E: \mathcal{G} \longrightarrow \mathcal{G}^{\prime}$ in $\operatorname{BGrb}(M)$ gives rise to a morphism of smooth 2-groups $\widehat{E}: \operatorname{Sym}_{G}(\mathcal{G}) \longrightarrow \operatorname{Sym}_{G}\left(\mathcal{G}^{\prime}\right)$ : choose an adjoint inverse $E^{\vee}$ for $E$ and define $\widehat{E}$ via

$$
(f, A) \longmapsto \Phi_{f}^{*} E \circ(f, A) \circ E^{\vee} .
$$

Proposition 5.46. There is a natural bijection $\Xi_{E}$ between the set $\mathcal{E}(E)$ of equivariant structures on $E$ and the set of 2-isomorphisms $\widehat{\gamma}: \widehat{E} \circ s \longrightarrow s^{\prime}$ of smooth morphisms of 2-groups.

Proof. Let $\mathcal{G}$ and $\mathcal{G}^{\prime}$ be $G$-equivariant bundle gerbes on a manifold $M$ with a smooth $G$-action $\Phi: G \times M \longrightarrow M$, and let $(E, \gamma): \mathcal{G} \longrightarrow \mathcal{G}^{\prime}$ be a 1-isomorphism of equivariant bundle gerbes. Fix an adjoint inverse $E^{\vee}$ to $E$. We construct the 2-isomorphism $\Xi_{E}(\gamma): \widehat{E} \circ s \longrightarrow s^{\prime}$ as follows: let $f: c \longrightarrow G$ be an element of $\underline{G}$. The natural transformation $\Xi_{E}(\gamma)$ consists of a 2-isomorphism $\Xi_{E}(\gamma)_{f}: \widehat{E} \circ s(f) \longrightarrow s^{\prime}(f)$ which we construct by the diagram

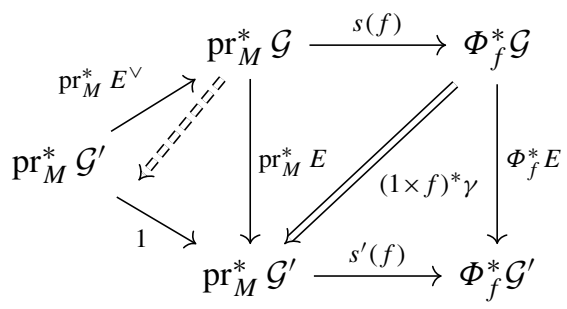


Using the same methods as in the proof of Theorem 5.42 one can show that $\Xi_{E}$ is a bijection.

Definition 5.47. An equivariant 2-isomorphism of $G$-equivariant bundle gerbes $(E, \gamma)$ $\longrightarrow\left(E^{\prime}, \gamma^{\prime}\right)$ consists of a 2-morphism $\eta: E \longrightarrow E^{\prime}$ such that there is an equality of diagrams

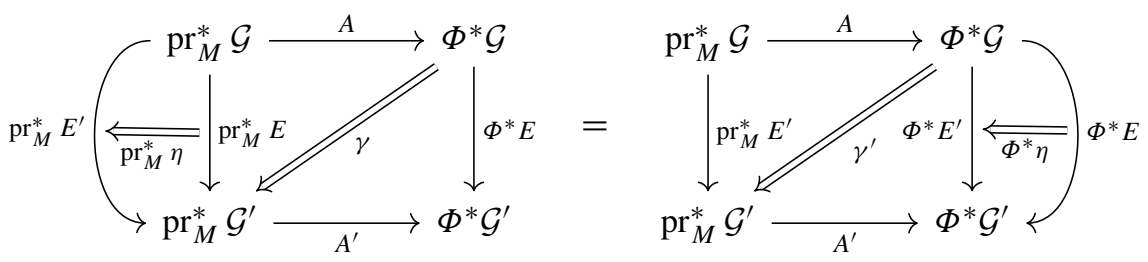

Being an equivariant 2-morphism is a property.

The 2-group extension $\operatorname{Sym}_{G}(\mathcal{G}) \longrightarrow \underline{G}$ can also be used to study the existence of equivariant structures on 1-morphisms. A condition for 2-isomorphisms of bundle gerbes to be equivariant is

Proposition 5.48. Let $(E, \gamma)$ and $\left(E^{\prime}, \gamma^{\prime}\right)$ be equivariant 1-isomorphisms. A 2isomorphism $\eta: E \longrightarrow E^{\prime}$ is equivariant if and only if $\Xi_{E^{\prime}}\left(\gamma^{\prime}\right) \circ\left(\widehat{\eta} \circ 1_{\gamma}\right)=\Xi_{E}(\gamma)$.

Proof. This follows from Definition 5.47 using the fact that the inverses $E^{\vee}$ and $E^{\prime \vee}$ are adjoints to $E$ and $E^{\prime}$.

\section{Application I: Nonassociative Magnetic Translations}

Nonassociativity in quantum mechanics has a long history dating back to foundational work on the theory in the 1930's. Its interest was revived in the 1980's with the realisation that the magnetic translation operators on the states of a charged particle moving in a magnetic monopole background generally form a nonassociative algebra [Jac85, GZ86]; see [Sza19a] for a mathematical introduction to the subject together with a survey and comparison of the various approaches to the quantisation of the pertinent twisted Poisson structures. The recent revived interest in these models has come about from their conjectural relevance to the low-energy dynamics of closed strings in non-geometric backgrounds, which are based on arguments invoking T-duality applied to target spaces that are tori or more generally total spaces of torus bundles [Lüs10,MSS12,BL14,MSS14], and other compact Lie groups [BP11]. See e.g. [Sza19b] for a contemporary introduction to the subject with further references.

As a first application of the general framework presented in this paper, we reformulate the well-known theory of magnetic translations for source-free magnetic fields in the language of Sect. 3. We then use the results of Sect. 5 to describe nonassociative magnetic translations, which were first studied from a geometric perspective in [BMS19] on $\mathbb{R}^{d}$. They were subsequently studied from a quantum field theory perspective in [Mic19], where generalisations from $\mathbb{R}^{d}$ to connected Lie groups are also considered. Here we will show that they are induced by a natural section of $\operatorname{Sym}_{G}(\mathcal{G}) \longrightarrow \underline{G}$ constructed using the parallel transport of Sect. 4. 
6.1. Magnetic translations on $\mathbb{T}^{d}$. Magnetic translations appear in the quantum mechanics of an electrically charged particle moving on a manifold $M$ in the presence of a magnetic field, which is given by a 2 -form $B \in \Omega^{2}(M)$. In the semi-classical Maxwell theory of electromagnetism, the 2 -form $B$ is closed and has integer periods. The first requirement $H=\mathrm{d} B=0$ is the statement that there are no magnetic monopoles. The second requirement is the Dirac charge quantisation condition which states that $B$ is the curvature of a connection on a hermitean line bundle $L$ over $M$. In Bloch theory (see e.g. [Gru00]), the line bundle $L$ is used in geometric quantisation of the shift of the canonical symplectic structure on the cotangent bundle $T^{*} M$ by the 2 -form $B$, so that the quantum Hilbert space of wavefunctions for the particle is $\mathcal{H}=\mathrm{L}^{2}(M ; L)$, the space of square-integrable sections of $L$. The (global) symmetry group $G$ of the particle acts on $M$, and one would like to promote the $G$-action to an action on the Hilbert space by linear operators. In quantum mechanics, this action on $\mathcal{H}$ is only required to define a projective representation of $G$. If $G$ acts via translations the resulting operators are called magnetic translations. The construction in Sect. 3 provides a universal mechanism to construct magnetic translations, which we will illustrate on the example of a $d$-dimensional torus $M=\mathbb{T}^{d}$. Magnetic translations on $\mathbb{T}^{d}$ have been studied in e.g. [Fio13,DGTS20] (for constant magnetic fields $B$ ), but our treatment is more general and also fits in with expectations from string theory.

Instead of working on $\mathbb{T}^{d}$ directly, we work equivariantly on the universal cover $\mathbb{R}^{d}$ by viewing $\mathbb{T}^{d}=\mathbb{R}^{d} / \mathbb{Z}^{d}$ as the quotient of the natural free action $\tau$ on $\mathbb{R}^{d}$ of the discrete subgroup $\mathbb{Z}^{d} \subset \mathbb{R}^{d}$ by translations. The corresponding projection $\pi: \mathbb{R}^{d} \longrightarrow \mathbb{T}^{d}$ is a surjective submersion. To describe line bundles on $\mathbb{T}^{d}$ we consider the diagram of manifolds

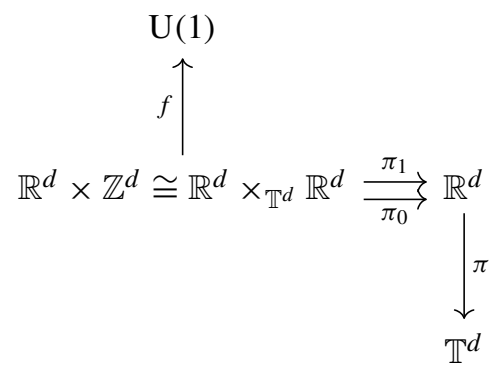

where we use the identification $\mathbb{R}^{d} \times \mathbb{Z}^{d} \ni(x, i) \longmapsto(x, x+i) \in \mathbb{R}^{d} \times_{\mathbb{T}^{d}} \mathbb{R}^{d}$; under this identification, $\pi_{0}=\mathrm{pr}_{\mathbb{R}^{d}}$ is the projection and $\pi_{1}=\tau$ is the $\mathbb{Z}^{d}$-action on $\mathbb{R}^{d}$. Any line bundle on $\mathbb{T}^{d}$ can be described by a smooth function $f: \mathbb{R}^{d} \times \mathbb{Z}^{d} \longrightarrow \mathrm{U}(1)$ satisfying

$$
f(x+i, j) f(x, i)=f(x, i+j)
$$

for all $x \in \mathbb{R}^{d}$ and $i, j \in \mathbb{Z}^{d}$. This means that $f$ is a 1 -cocycle on the group $\mathbb{Z}^{d}$ with values in the $\mathbb{Z}^{d}$-module $C^{\infty}\left(\mathbb{R}^{d} ; \mathrm{U}(1)\right)$. We will use the notation $f_{i}(\cdot):=f(\cdot, i) \in$ $C^{\infty}\left(\mathbb{R}^{d} ; \mathrm{U}(1)\right)$ for $i \in \mathbb{Z}^{d}$. Concretely, the U(1)-bundle described by $f$ is the quotient

$$
P_{f}:=\left(\mathbb{R}^{d} \times \mathrm{U}(1)\right) / \sim
$$

by the equivalence relation

$$
(x+i, 1) \sim\left(x, f_{i}(x)\right)
$$


for all $x \in \mathbb{R}^{d}$ and $i \in \mathbb{Z}^{d}$. Sections of the associated line bundle $L_{f} \longrightarrow \mathbb{T}^{d}$ are in one-to-one correspondence with equivariant functions on the universal covering space, which are functions $\psi \in C^{\infty}\left(\mathbb{R}^{d} ; \mathbb{C}\right)$ satisfying quasi-periodic boundary conditions

$$
\psi(x+i)=f_{i}(x) \psi(x) \text {. }
$$

The action of the translation group $G=\mathbb{R}_{\mathrm{t}}^{d}$ on $\mathbb{R}^{d}, x \longmapsto x+v$, induces an action $\tau$ of $\mathbb{R}_{\mathrm{t}}^{d}$ on $\mathbb{T}^{d}$. For $v \in \mathbb{R}_{\mathrm{t}}^{d}$, the bundle $\tau_{v}^{*} P_{f}$ is described by the functions $\tau_{v}^{*} f_{i}=f_{i}(\cdot-v) .{ }^{5}$ This allows us to give a concrete description for the fibres of the principal bundle $\operatorname{Sym}_{\mathbb{R}_{\mathrm{t}}^{d}}\left(P_{f}\right) \longrightarrow \mathbb{R}_{\mathrm{t}}^{d}$ as

$$
\begin{aligned}
\operatorname{Sym}_{\mathbb{R}_{\mathrm{t}}^{d}}\left(P_{f}\right)_{\mid v} & =\operatorname{Bun}_{\mathrm{U}(1)}(M)\left(P_{f}, P_{\tau_{v}^{*} f}\right) \\
& =\left\{g \in C^{\infty}\left(\mathbb{R}^{d}, \mathrm{U}(1)\right) \mid g(x+i)=f_{i}(x) f_{i}(x-v)^{-1} g(x)\right\} .
\end{aligned}
$$

The group $C^{6} C^{\infty}\left(\mathbb{T}^{d} ; \mathrm{U}(1)\right)=C^{\infty}\left(\mathbb{R}^{d} ; \mathrm{U}(1)\right)^{\mathbb{Z}^{d}}$ acts freely and transitively on $\mathrm{Sym}_{\mathbb{R}_{t}^{d}}$ $\left(P_{f}\right)_{\mid v}$ by pointwise multiplication. The multiplicative structure from Proposition 3.4 on $\operatorname{Sym}_{\mathbb{R}_{\mathrm{t}}^{d}}\left(P_{f}\right)$ takes the concrete form

$$
\mu\left((g, v),\left(g^{\prime}, v^{\prime}\right)\right):=\left(\left(\tau_{v^{\prime}}^{*} g\right) g^{\prime}, v+v^{\prime}\right) .
$$

A smooth section of the short exact sequence

$$
1 \longrightarrow C^{\infty}\left(\mathbb{T}^{d}, \mathrm{U}(1)\right) \longrightarrow \operatorname{Sym}_{\mathbb{R}_{\mathrm{t}}^{d}}\left(P_{f}\right) \longrightarrow \mathbb{R}_{\mathrm{t}}^{d} \longrightarrow 1
$$

induces a twisted action of $\mathbb{R}_{t}^{d}$ on the quantum Hilbert space $\mathcal{H}=\mathrm{L}^{2}\left(\mathbb{T}^{d} ; L_{f}\right)$; here we do not require this section to be a group homomorphism, and the 2-cocycle twisting this action takes values in $C^{\infty}\left(\mathbb{T}^{d} ; \mathrm{U}(1)\right)$. We can construct such a section from the choice of a connection on $P_{f}$, which reproduces the usual expression for magnetic translations. A connection on $P_{f}$ can be described by a 1 -form $A \in \Omega^{1}\left(\mathbb{R}^{d}\right)$ satisfying

$$
-\mathrm{id} \log f=\pi_{0}^{*} A-\pi_{1}^{*} A .
$$

This condition implies that the closed 2-form $\mathrm{d} A=\pi^{*} B$ descends to a well-defined magnetic field $B$ on $\mathbb{T}^{d}$. The section corresponding to $A$ is given by parallel transport:

$$
\begin{aligned}
s_{A}: \mathbb{R}_{\mathrm{t}}^{d} \longrightarrow \operatorname{Sym}_{\mathbb{R}_{\mathrm{t}}^{d}}\left(P_{f}\right) & \\
v & \longmapsto s_{A}(v)=\exp \left(-\mathrm{i} \int_{\Delta^{1}(\cdot ; v)} A\right),
\end{aligned}
$$

where

$$
\Delta^{1}(x ; v)=\left\{x-v+t v \in \mathbb{R}^{d} \mid 0 \leq t \leq 1\right\} .
$$

\footnotetext{
${ }^{5}$ Note that the functions $f_{i}$ are not invariant under the subgroup $\mathbb{Z}_{\mathrm{t}}^{d} \subset \mathbb{R}_{\mathrm{t}}^{d}$, whereas the bundles $P_{f}$ and $P_{\tau_{v}^{*} f}$ are canonically isomorphic. This is nothing but a concrete implementation of the fact that pullbacks are only well defined up to canonical isomorphism.

${ }^{6}$ For an action of a group $G$ on a set $X$, we denote by $X^{G} \subseteq X$ the subset of $G$-invariants.
} 
We check that this is indeed an element of $\operatorname{Sym}_{\mathbb{R}_{\mathrm{t}}^{d}}\left(P_{f}\right)_{\mid v}$ :

$$
\begin{aligned}
\left(s_{A}(v)\right)(x+i) & =\exp \left(-\mathrm{i} \int_{\Delta^{1}(x+i ; v)} A+\mathrm{i} \int_{\Delta^{1}(x ; v)} A\right)\left(s_{A}(v)\right)(x) \\
& =\exp \left(\int_{\Delta^{1}(x ; v)} \mathrm{d} \log f_{i}\right)\left(s_{A}(v)\right)(x) \\
& =f_{i}(x) f_{i}(x-v)^{-1}\left(s_{A}(v)\right)(x) .
\end{aligned}
$$

The corresponding 2-cocycle describing the extension agrees with the 2-cocycle constructed in e.g. [Sol18, BMS19].

By Proposition 3.5 the extension $\operatorname{Sym}_{\mathbb{R}_{t}^{d}}\left(P_{f}\right)$ acts on the total space of the line bundle $L_{f}$ and hence on the quantum state space $\mathcal{H}$. The section $s_{A}$ realises translations $v \in \mathbb{R}_{\mathrm{t}}^{d}$ as linear operators $\mathcal{P}(v): \mathcal{H} \longrightarrow \mathcal{H}$ on this Hilbert space via

$$
\mathcal{P}(v) \psi=s_{A}(v) \tau_{v}^{*} \psi
$$

One easily checks that $\mathcal{P}(v) \psi \in \mathcal{H}$ for $\psi \in \mathcal{H}$, i.e. $(\mathcal{P}(v) \psi)(x+i)=f_{i}(x)(\mathcal{P}(v) \psi)(x)$. However, they only provide a projective representation of the translation group $\mathbb{R}_{t}^{d}$ since $s_{A}$ is not a group homomorphism. Explicitly, using Stokes' Theorem we find that the magnetic translations satisfy the relations of the twisted group algebra

$$
\mathcal{P}(v) \mathcal{P}\left(v^{\prime}\right)=\exp \left(-\mathrm{i} \int_{\Delta^{2}\left(\cdot ; v^{\prime}, v\right)} \pi^{*} B\right) \mathcal{P}\left(v+v^{\prime}\right),
$$

where

$$
\Delta^{2}\left(x ; v^{\prime}, v\right)=\left\{x-v-v^{\prime}+t_{1} v^{\prime}+t_{2} v \in \mathbb{R}^{d} \mid 0 \leq t_{2} \leq t_{1} \leq 1\right\},
$$

and we used the relation

$$
\partial \Delta^{2}\left(x ; v^{\prime}, v\right)=\Delta^{1}(x ; v)-\Delta^{1}\left(x ; v+v^{\prime}\right)+\Delta^{1}\left(x+v ; x+v+v^{\prime}\right)
$$

in the simplicial complex in $\mathbb{R}^{d}$.

Remark 6.1. By dropping the (quasi-)periodicity conditions everywhere one gets back the description of magnetic translations corresponding to (necessarily trivialisable) line bundles over $\mathbb{R}^{d}$ (cf. [BMS19]).

6.2. Nonassociative magnetic translations from parallel transport. Dirac's extension of the classical Maxwell theory assumes a singular magnetic field $B$ whose 3-form curvature $H=\mathrm{d} B$ is distributional, with zero-dimensional support consisting of the locations of magnetic monopoles on the configuration manifold $M$. However, in applications to string theory the closed 3 -form $H$ corresponds to an NS-NS flux and is typically smooth, as we now assume. The framework described in Sect. 6.1 is not capable of encoding magnetic fields with non-vanishing magnetic charge $H=\mathrm{d} B$, since in this case $B$ can never be realised as the curvature of a line bundle. The quantisation problem now concerns an $H$-twisted Poisson structure on the cotangent bundle $T^{*} M$ [Sza19a], with twisting of the canonical Poisson structure which spoils the Jacobi identity for functions in $C^{\infty}\left(T^{*} M ; \mathbb{C}\right)$ that vary along the vertical directions. The corresponding quantum operators do not associate; it is not possible to realise a nonassociative algebra by linear 
operators acting on a Hilbert space. Different approaches to describing the nonassociative quantum mechanics of charged particles moving in the background of a magnetic field with smooth monopole sources are described in [MSS14, BBBS15, KS18].

Fluxes in string theory obey a generalised version of Dirac charge quantisation (see e.g. [Sza13]); in particular, the closed 3-form $H$ has integer periods and hence is the curvature of a connection on a hermitean bundle gerbe over $M$. Based on this observation, in [BMS19] we suggested the following approach: geometrically the magnetic field $B$ can be interpreted as the curving on a trivial gerbe $\mathcal{I}_{B}$ with curvature $H$. We proposed to use the 2-Hilbert space of sections of $\mathcal{I}_{B}$ as a replacement for the usual Hilbert space of quantum mechanics. The category of sections of a gerbe $\mathcal{G}$ on a manifold $M$ is the morphism category $\Gamma(M ; \mathcal{G}):=\operatorname{BGrb}(\mathcal{I}, \mathcal{G})$; for details on the 2-Hilbert space structure on this category we refer to [BSS18,BS17,Bun17]. As evidence for our approach we constructed a projective action of nonassociative magnetic translation operators on this 2Hilbert space, which naturally encodes the relations of the $H$-twisted Poisson algebra on $T^{*} M$. However, the drawback of this approach is that it does not work for topologically non-trivial fluxes $H$, or equivalently for gerbes $\mathcal{G}$ with non-torsion Dixmier-Douady class. Extending our geometric approach to nonassociative quantum mechanics along these lines was in fact one of our original motivations behind the present paper.

Let us first explain how the action of nonassociative magnetic translations for magnetic fields with sources on $M=\mathbb{R}^{d}$, which was described in [BMS19], can be constructed via the 2-group extensions from Sect. 5. Every gerbe on $\mathbb{R}^{d}$ is isomorphic to a trivial gerbe $\mathcal{I}_{B}$ represented by the diagram

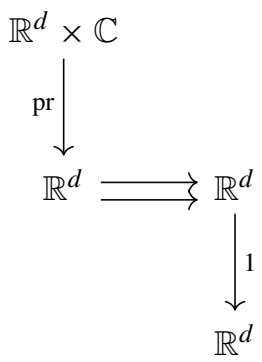

with trivial connection $A=0$ and curving $B \in \Omega^{2}\left(\mathbb{R}^{d}\right)$. The connection on $\mathcal{I}_{B}$ induces a section ${ }^{7}$

$$
s_{B}: \mathbb{R}_{\mathrm{t}}^{d} \longrightarrow \operatorname{Sym}_{\mathbb{R}_{\mathrm{t}}^{d}}(\mathcal{I})
$$

via parallel transport:

$$
s_{B}(v):=\left(\mathrm{pt}_{1}^{\mathcal{I}_{B}}\right)_{\mid \Delta^{1}(\cdot ; v)}: \mathcal{I}_{B} \longrightarrow \tau_{v}^{*} \mathcal{I}_{B}
$$

Combining $s_{B}$ with the action of $\operatorname{Sym}_{\mathbb{R}_{\mathrm{t}}^{d}}(\mathcal{I})$ on $\mathcal{I}$ induces a higher projective action of $\mathbb{R}_{\mathrm{t}}^{d}$ on $\Gamma\left(\mathbb{R}^{d} ; \mathcal{I}\right)$. We refer to [BMS19, Section 4] for precise definitions.

Since all line bundles over $\mathbb{R}^{d}$ are isomorphic to a trivial line bundle, the category $\operatorname{Sym}_{\mathbb{R}_{\mathrm{t}}^{d}}(\mathcal{I})_{\mid v}$ at $v \in \mathbb{R}_{\mathrm{t}}^{d}$ is equivalent to the category with one object and morphisms

\footnotetext{
7 To simplify the presentation, in the following we disregard smooth structures and work in the 2-category of 2-groups $2 \mathcal{G r p}(\mathcal{G r p d})$. The extensions to categories fibred in groupoids over Cart is straightfoward.
} 
described by smooth functions $\mathbb{R}^{d} \longrightarrow \mathrm{U}(1)$. Thus

$$
\operatorname{Sym}_{\mathbb{R}_{\mathrm{t}}^{d}}(\mathcal{I}) \cong\left(* / / C^{\infty}\left(\mathbb{R}^{d} ; \mathrm{U}(1)\right)\right) \rtimes \mathbb{R}_{\mathrm{t}}^{d} .
$$

The 2-group structure on $\operatorname{Sym}_{\mathbb{R}_{t}^{d}}(\mathcal{I})$ is given by

$$
\begin{aligned}
v \otimes v^{\prime} & :=v+v^{\prime}, \\
(h, v) \otimes\left(h^{\prime}, v^{\prime}\right) & :=\left(\left(\tau_{v^{\prime}}^{*} h\right) h^{\prime}, v+v^{\prime}\right) .
\end{aligned}
$$

However, under this equivalence the action of the magnetic translation operators becomes elusive. In [BMS19] we equipped the 1-morphisms with a connection to circumvent this problem. We did not require the 2-morphisms to preserve these connections leading to equivalent categories. The parallel transport 1-morphisms $p t_{1}^{\mathcal{I}_{B}}$ can be equipped with such a connection in a canonical way. This reproduces the constructions from [BMS19].

For this, let $\mathcal{I}_{B}$ be a trivial bundle gerbe on a smooth manifold $M$ with curving $B \in \Omega^{2}(M)$ and curvature $H=\mathrm{d} B$. We fix a base point $x \in M$. Via transgression and regression we get a bundle gerbe $\mathcal{R} \mathcal{T}\left(\mathcal{I}_{B}\right)$ defined over the diffeological path fibration $P_{0} M \longrightarrow M$. The corresponding line bundle over $\left(P_{0} M\right)^{[2]}$ admits a canonical trivialisation. It comes equipped with a connection 1-form given as the pullback of the transgression of $B$ to the loop space $L M$ along the embedding $l:\left(P_{0} M\right)^{[2]} \longrightarrow L M$. To describe the curving of $\mathcal{R} \mathcal{T}\left(\mathcal{I}_{B}\right)$ we note that a tangent vector to a based path $\gamma \in P_{0} M$ is a smooth section $\mathcal{V} \in \Gamma\left([0,1] ; \gamma^{*} T M\right)$ which is zero in a neighbourhood of 0 and constant in a neighbourhood of 1 . The 2 -form $\mathcal{R T}(B) \in \Omega^{2}\left(P_{0} M\right)$ is defined by the transgression formula

$$
\mathcal{R} \mathcal{T}(B)\left(\mathcal{V}, \mathcal{V}^{\prime}\right)=\int_{\gamma} \iota^{\prime}{ }^{\prime} \mathcal{V} H,
$$

where $l$ denotes the interior multiplication between a vector and a form. There is a natural 1-isomorphism [Wal16, Section 6.1] $W: \mathcal{R} \mathcal{T}\left(\mathcal{I}_{B}\right) \longrightarrow \mathcal{I}_{B}$ with underlying diagram

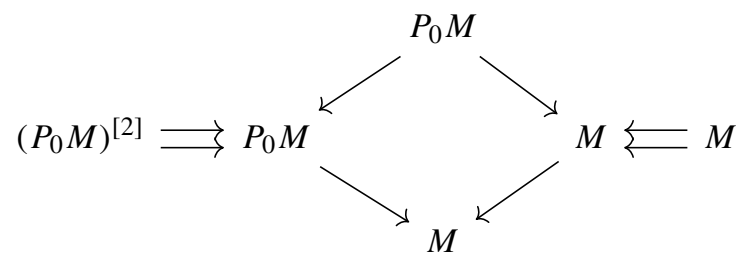

of diffeological spaces. The line bundle which is part of this 1-morphism has a canonical trivialisation for trivial bundle gerbes $\mathcal{I}_{B}$ and has the connection 1-form $A_{W} \in \Omega^{1}\left(P_{0} M\right)$ defined by the transgression formula

$$
A_{W}(\mathcal{V})=\int_{\gamma}{ }^{l} \mathcal{V} B
$$

In order to describe the parallel transport, we pull everything back to the path space $P M$ along the two evaluation maps $\mathrm{ev}_{0}, \mathrm{ev}_{1}: P M \longrightarrow M$. The parallel transport defined in Sect. 4 is a 1-morphism

$$
\mathrm{pt}_{1}^{\mathcal{R T}\left(\mathcal{I}_{B}\right)}: \mathrm{ev}_{0}^{*} \mathcal{R} \mathcal{T}\left(\mathcal{I}_{B}\right) \longrightarrow \mathrm{ev}_{1}^{*} \mathcal{R} \mathcal{T}\left(\mathcal{I}_{B}\right)
$$


given by a line bundle with connection over the space $P_{\partial \Delta^{2}} M$. An element of $P_{\partial \Delta^{2}} M$ is a triple of paths $\left(\gamma_{x y}, \gamma_{y z}, \gamma_{x z}\right)$, where $\gamma_{x y}$ is a path from the base point $x$ to some other point $y \in M, \gamma_{y z}$ is a path from $y$ to a third point $z \in M$, and $\gamma_{x z}$ is a path from $x$ to $z$. Again, the line bundle for the parallel transport is trivial since we work with a trivial bundle gerbe. Its connection is given by

$$
\int_{\overline{\gamma_{x z}} \star \gamma_{y z} \star \gamma_{x y}} l_{\bullet} B,
$$

where the notation means that the evaluation on a tangent vector $\mathcal{V}$ is given by

$$
\int_{\overline{\gamma_{x z}} \star \gamma_{y z} \star \gamma_{x y}} l \mathcal{V} B .
$$

We obtain a 1-morphism

$$
\mathrm{pt}_{1}^{\mathcal{I}_{B}}: \mathrm{ev}_{0}^{*} \mathcal{I}_{B} \stackrel{\mathrm{ev}_{0}^{*} W^{-1}}{\longrightarrow} \mathrm{ev}_{0}^{*} \mathcal{R} \mathcal{T}\left(\mathcal{I}_{B}\right) \stackrel{\mathrm{pt}_{1}^{\mathcal{R} \mathcal{T}\left(\mathcal{I}_{B}\right)}}{\longrightarrow} \mathrm{ev}_{1}^{*} \mathcal{R} \mathcal{T}\left(\mathcal{I}_{B}\right) \stackrel{\mathrm{ev}_{1}^{*} W}{\longrightarrow} \mathrm{ev}_{1}^{*} \mathcal{I}_{B}
$$

representing the colimit from Definition 4.16. Concretely, this is a trivial line bundle over $P M$ with connection

$$
\int_{\gamma_{y z}} l_{\bullet} B .
$$

This is exactly the formula used for the magnetic translations in [BMS19] in the case $M=\mathbb{R}^{d}$, hence it provides a conceptual underpinning of the constructions in [BMS19], and moreover generalises them to trivial bundle gerbes on arbitrary manifolds $M$.

6.3. Nonassociative magnetic translations on $\mathbb{T}^{d}$. Now we generalise the description of nonassociative magnetic translations to the $d$-dimensional torus $\mathbb{T}^{d}$, see also [Mic19] for a discussion from a quantum field theory point of view. A problem in this context is that for topologically non-trivial gerbes on $\mathbb{T}^{d}$, there are no non-trivial sections. This makes the 2-Hilbert space of sections an uninteresting object to study. ${ }^{8}$ However, our 2group extension still exists and should encode the geometry of nonassociative magnetic translations in this context, regardless of whether or not sections exist. Non-trivial gerbes over $\mathbb{T}^{d}$ are similarly treated as coming from $\mathbb{Z}^{d}$-equivariant (topologically trivial) gerbes over $\mathbb{R}^{d}$, as in e.g. [MW16, Section 7.1].

Bundle gerbes on $\mathbb{T}^{d}$ can be described using the surjective submersion $\pi: \mathbb{R}^{d} \longrightarrow \mathbb{T}^{d}$ and the corresponding diagram

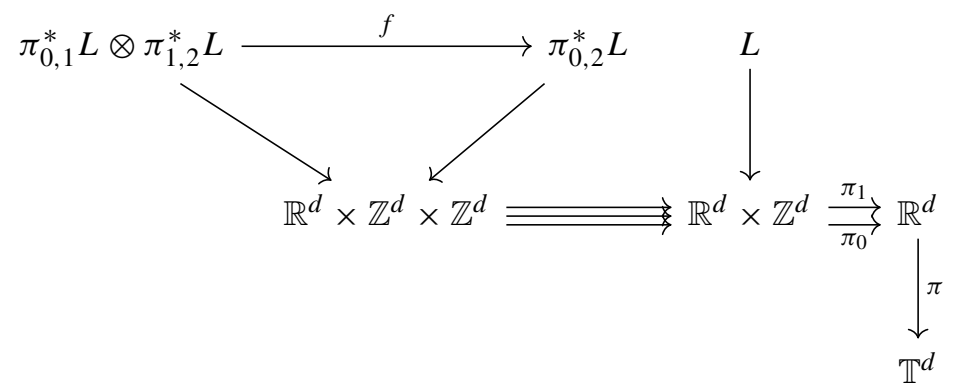

8 We expect that there exists a better definition of sections circumventing this problem. 
Here we used the identification $(x, i, j) \in \mathbb{R}^{d} \times \mathbb{Z}^{d} \times \mathbb{Z}^{d} \longmapsto(x, x+i, x+i+j) \in\left(\mathbb{R}^{d}\right)^{[3]}$. Concretely, a bundle gerbe consists of a line bundle $L$ over $\mathbb{R}^{d} \times \mathbb{Z}^{d}$, which we can assume to be trivial without loss of generality, and an isomorphism $f: \pi_{0,1}^{*} L \otimes \pi_{1,2}^{*} L \longrightarrow \pi_{0,2}^{*} L$ of line bundles over $\mathbb{R}^{d} \times \mathbb{Z}^{d} \times \mathbb{Z}^{d}$ satisfying a coherence condition over $\mathbb{R}^{d} \times\left(\mathbb{Z}^{d}\right)^{\times 3}$. We can describe this isomorphism by a collection of smooth maps $f_{i, j}: \mathbb{R}^{d} \longrightarrow \mathrm{U}(1)$ for all $i, j \in \mathbb{Z}^{d}$, and the coherence condition translates to

$$
f_{i, j}(x) f_{i+j, k}(x)=f_{i, j+k}(x) f_{j, k}(x+i)
$$

for all $x \in \mathbb{R}^{d}$ and $i, j, k \in \mathbb{Z}^{d}$, which is the 2-cocycle condition for

$$
f \cdot, \cdot \in \mathrm{C}^{2}\left(\mathbb{Z}^{d} ; C^{\infty}\left(\mathbb{R}^{d} ; \mathrm{U}(1)\right)\right) .
$$

We denote the gerbe described by $f$ as $\mathcal{G}_{f}$.

For $v \in \mathbb{R}_{\mathrm{t}}^{d}$, the pullback of $\mathcal{G}_{f}$ along the translation $\tau_{v}$ can be described by the 2-cocycle $\tau_{v}^{*} f_{i, j}=f_{i, j}(\cdot-v)$. Using [Bun17, Proposition A.31] we can describe the category $\operatorname{Sym}_{\mathbb{R}_{\mathrm{t}}^{d}}\left(\mathcal{G}_{f}\right)_{\mid v}$ at $v \in \mathbb{R}_{\mathrm{t}}^{d}$ up to equivalence in the following way: its objects are functions $g: \mathbb{R}^{d} \times \mathbb{Z}^{d} \longrightarrow \mathrm{U}(1)$ satisfying

$$
f_{i, j}(x-v) g_{i}(x) g_{j}(x+i)=g_{i+j}(x) f_{i, j}(x),
$$

for all $x \in \mathbb{R}^{d}$ and $i, j \in \mathbb{Z}^{d}$. It is straightforward to deduce the morphisms in $\operatorname{Sym}_{\mathbb{R}_{\mathrm{t}}^{d}}\left(\mathcal{G}_{f}\right)$ from [Bun17, Proposition A.31]; we find that a morphism from $g$ to $g^{\prime}$ is described by a function $h: \mathbb{R}^{d} \longrightarrow \mathrm{U}(1)$ satisfying

$$
h(x) g_{i}(x)=g_{i}^{\prime}(x) h(x+i)
$$

for all $x \in \mathbb{R}^{d}$ and $i \in \mathbb{Z}^{d}$. Note that for the trivial 2-cocycle $f_{i, j}=1$ this describes the category of line bundles over $\mathbb{T}^{d}$ with arbitrary gauge transformations as morphisms. The 2-group structure on

$$
\operatorname{Sym}_{\mathbb{R}_{\mathrm{t}}^{d}}\left(\mathcal{G}_{f}\right)=\coprod_{v \in \mathbb{R}_{\mathrm{t}}^{d}} \operatorname{Sym}_{\mathbb{R}_{\mathrm{t}}^{d}}\left(\mathcal{G}_{f}\right)_{\mid v}
$$

from Theorem 5.23 takes the form

$$
\begin{gathered}
\left(g_{i}, v\right) \otimes\left(g_{i}^{\prime}, v^{\prime}\right):=\left(\left(\tau_{v^{\prime}}^{*} g_{i}\right) g_{i}^{\prime}, v+v^{\prime}\right), \\
(h, v) \otimes\left(h^{\prime}, v^{\prime}\right):=\left(\left(\tau_{v^{\prime}}^{*} h\right) h^{\prime}, v+v^{\prime}\right),
\end{gathered}
$$

fitting into the 2-group extension from Theorem 5.27:

$$
1 \longrightarrow \operatorname{HLBdl}\left(\mathbb{T}^{d}\right) \longrightarrow \operatorname{Sym}_{\mathbb{R}_{\mathrm{t}}^{d}}\left(\mathcal{G}_{f}\right) \longrightarrow \mathbb{R}_{\mathrm{t}}^{d} \longrightarrow 1
$$

As in the case of line bundles from Sect. 6.1, a connection $(A, B)$ on $\mathcal{G}_{f}$ induces a section of the extension (6.2). A connection on $\mathcal{G}_{f}$ is described by a 2-form $B \in \Omega^{2}\left(\mathbb{R}^{d}\right)$ and a 1 -form $A \in \Omega^{1}\left(\mathbb{R}^{d} \times \mathbb{Z}^{d}\right)$ satisfying

$$
\begin{aligned}
-\mathrm{id} \log f_{i, j}(x) & =A_{i+j}(x)-A_{i}(x)-A_{j}(x+i), \\
\mathrm{d} A & =\pi_{1}^{*} B-\pi_{0}^{*} B,
\end{aligned}
$$


for all $x \in \mathbb{R}^{d}$ and $i, j \in \mathbb{Z}^{d}$. The second condition implies that the closed 3-form $\mathrm{d} B=\pi^{*} H$ descends to a well-defined flux $H$ on $\mathbb{T}^{d}$. Using the connection we can construct a section

$$
s_{A, B}: \mathbb{R}_{\mathrm{t}}^{d} \longrightarrow \operatorname{Sym}_{\mathbb{R}_{\mathrm{t}}^{d}}\left(\mathcal{G}_{f}\right)
$$

by

$$
s_{A, B}(v):=\left(g_{i}, v\right) \quad \text { with } \quad g_{i}=\exp \left(\mathrm{i} \int_{\Delta^{1}(\cdot ; v)} A_{i}\right) .
$$

We check that this is indeed an element of $\operatorname{Sym}_{\mathbb{R}_{\mathrm{t}}^{d}}\left(\mathcal{G}_{f}\right)_{\mid v}$ :

$$
\begin{aligned}
g_{i}(x) g_{j}(x+i) g_{i+j}(x)^{-1} & =\exp \left(\mathrm{i} \int_{\Delta^{1}(x ; v)} A_{i}+\mathrm{i} \int_{\Delta^{1}(x+i ; v)} A_{j}-\mathrm{i} \int_{\Delta^{1}(x ; v)} A_{i+j}\right) \\
& =\exp \left(-\int_{\Delta^{1}(x ; v)} \mathrm{d} \log f_{i, j}\right) \\
& =f_{i, j}(x-v)^{-1} f_{i, j}(x)
\end{aligned}
$$

For the multiplication we find

$$
\begin{aligned}
s_{A, B}(v) \otimes s_{A, B}\left(v^{\prime}\right) & =\left(\exp \left(\mathrm{i} \int_{\Delta^{1}\left(\cdot-v^{\prime} ; v\right)} A_{i}+\mathrm{i} \int_{\Delta^{1}\left(\cdot ; v^{\prime}\right)} A_{i}\right), v+v^{\prime}\right) \\
& =\left(\exp \left(\mathrm{i} \int_{\Delta^{1}\left(\cdot ; v+v^{\prime}\right)} A_{i}-\mathrm{i} \int_{\Delta^{2}\left(\cdot ; v^{\prime}, v\right)}\left(B-\tau_{i}^{*} B\right)\right), v+v^{\prime}\right) \\
& =s_{A, B}\left(v+v^{\prime}\right) \otimes\left(\exp \left(-\mathrm{i} \int_{\Delta^{2}\left(\cdot ; v^{\prime}, v\right)}\left(B-\tau_{i}^{*} B\right)\right), 0\right) .
\end{aligned}
$$

This particular product is associative on the nose. However, the line bundle on $\mathbb{T}^{d}$ described by the transition functions $\exp \left(-\mathrm{i} \int_{\Delta^{2}\left(\cdot ; v^{\prime}, v\right)}\left(B-\tau_{i}^{*} B\right)\right)$ is non-trivial. We can use the decomposition

$$
\int_{\Delta^{2}\left(\cdot ; v^{\prime}, v\right)} \mathrm{d} l_{\bullet} B=£_{\bullet} \int_{\Delta^{2}\left(\cdot ; v^{\prime}, v\right)} B-\int_{\Delta^{2}\left(\cdot ; v^{\prime}, v\right)} l_{\bullet} \pi^{*} H,
$$

where $£$ is the Lie derivative, to construct a 2 -isomorphism

$$
\Pi_{v, v^{\prime}}:=\exp \left(-\mathrm{i} \int_{\Delta^{2}\left(\cdot ; v^{\prime}, v\right)} B\right): s_{A, B}(v) \otimes s_{A, B}\left(v^{\prime}\right) \longrightarrow s_{A, B}\left(v+v^{\prime}\right),
$$

which has the advantage that the line bundle on the right-hand side is trivial.

Remark 6.3. In this last representation the nonassociativity of the higher magnetic translations is realised by the composition property

$$
\Pi_{u, v+w} \circ \tau_{-u}^{*} \Pi_{v, w}=\omega_{u, v, w} \Pi_{u+v, w} \circ \Pi_{u, v},
$$

where

$$
\omega_{u, v, w}=\exp \left(\mathrm{i} \int_{\Delta^{3}(\cdot ; w, v, u)} \pi^{*} H\right)
$$


and

$$
\Delta^{3}(x ; w, v, u)=\left\{x-u-v-w+t_{1} w+t_{2} v+t_{3} u \in \mathbb{R}^{d} \mid 0 \leq t_{3} \leq t_{2} \leq t_{1} \leq 1\right\} .
$$

Concretely this means that there are two different ways to go from the triple product $s_{A, B}(u) \otimes s_{A, B}(v) \otimes s_{A, B}(w)$ to $s_{A, B}(u+v+w)$. Their difference is controlled by the 3 -cocycle $\omega_{., .},$. on the translation group $\mathbb{R}_{\mathrm{t}}^{d}$ with values in $C^{\infty}\left(\mathbb{T}^{d} ; \mathrm{U}(1)\right)$, as depicted in the commutative diagram

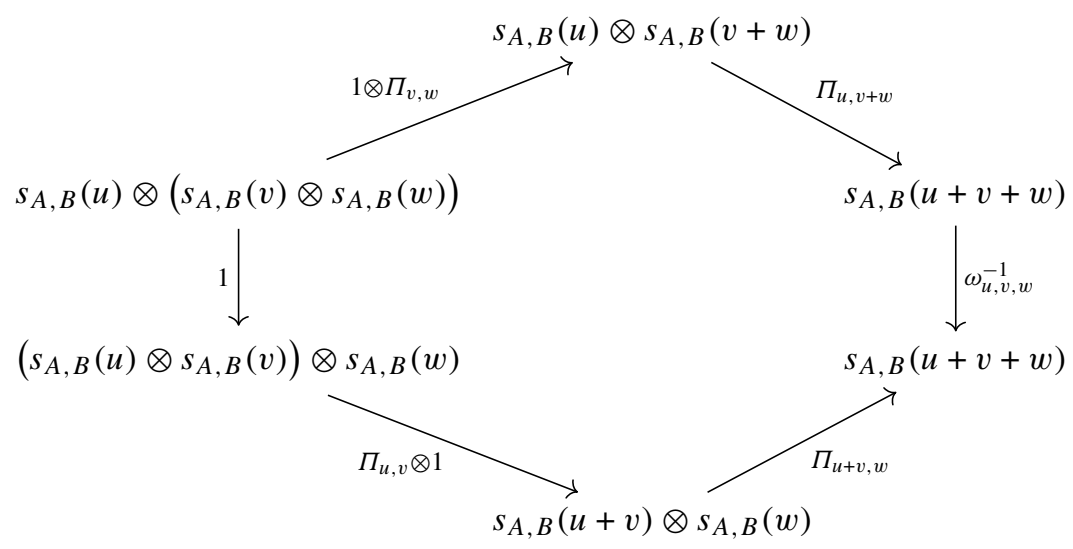

This is the implementation of nonassociativity in the higher categorical framework.

\section{Application II: Anomalies in Quantum Field Theory}

In this section we begin by using the group extensions $\operatorname{Sym}_{G}(P)$ from Sect. 3 to study the existence of equivariant trivialisations of line bundles. This has direct applications to the path integral description of the chiral anomaly in quantum field theory on evendimensional spacetime manifolds. Then using the 2-group extension $\operatorname{Sym}_{G}(\mathcal{G})$ from Sect. 5, we study the analogous questions for gerbes and apply our findings to the Hamiltonian description of the chiral anomaly on odd-dimensional time-slices.

7.1. Even dimensions: chiral anomalies. Let $G$ be a connected Lie group, $M$ a manifold with smooth $G$-action $\Phi: G \times M \longrightarrow M$, and $(P, \chi)$ a $G$-equivariant $\mathrm{U}(1)$-bundle on $M$. The equivariant structure on $P$ can be described by a splitting $s_{P}: G \longrightarrow \operatorname{Sym}_{G}(P)$. Assume furthermore that $P$ is trivial as a line bundle, i.e. there exists a 1-isomorphism $\psi: I \longrightarrow P$. The trivial bundle carries a canonical equivariant structure with corresponding splitting $s_{I}: G \longrightarrow \operatorname{Sym}_{G}(I)$.

Rewriting the result of Sect. 3.2 slightly, we see that $\psi$ is equivariant if and only if the smooth 1-cocycle

$$
\begin{aligned}
G \longrightarrow C^{\infty}(M ; \mathrm{U}(1)) \\
g \longmapsto s_{P}(g) \widehat{\psi}\left(s_{I}(g)\right)^{-1}
\end{aligned}
$$

is trivial. Every other isomorphism $I \longrightarrow P$ differs from $\psi$ by a uniquely determined element of $C^{\infty}(M ; \mathrm{U}(1))$. Their corresponding 1-cocycles differ by the coboundary 
defined by this element. Hence the obstruction for an equivariant bundle which is trivial as a line bundle to be also trivial as an equivariant bundle is an element of the degree one group cohomology $\mathrm{H}^{1}\left(G ; C^{\infty}(M ; \mathrm{U}(1))\right)$. This has also been observed in [CM95] from a different perspective.

The question of whether a bundle is equivariantly trivial is important in the path integral perspective on chiral anomalies in quantum field theory. Let $M$ be a based evendimensional compact Riemannian spin manifold, $G$ a Lie group, $Q$ a principal $G$-bundle on $M$, and $\rho: G \longrightarrow$ End $(V)$ a unitary representation of $G$ on a finite-dimensional vector space $V$ which encodes the matter content of the field theory. Denote by $S^{+}$and $S^{-}$the positive and negative chirality spinor bundles on $M$, respectively, by $\Gamma$ the group of based gauge transformations of $Q$ and by $\mathcal{A}$ the affine space of connections on $Q$. The field content of the theory are chiral spinors, which are smooth sections of the vector bundle $S^{+} \otimes V$, where here $V$ is the hermitean vector bundle associated to $Q$ via the representation $\rho$. There is a family of (twisted) Dirac operators

$$
\not D_{A}: \Gamma\left(M ; S^{+} \otimes V\right) \longrightarrow \Gamma\left(M ; S^{-} \otimes V\right)
$$

parameterised by gauge fields $A \in \mathcal{A}$, which are first order elliptic differential operators acting on chiral spinors. These data together define the content of a chiral gauge theory.

The formal path integral over the chiral spinor fields is the determinant $\operatorname{det}\left(\not_{A}\right)$ of the Dirac operator $D_{A}$. However, the determinant of $\not_{A}$ is in general not a number but an element of a complex line, and it can be defined only after suitable regularisation as an element of the fibre of the determinant line bundle det $\longrightarrow \mathcal{A}$ [AS84]. This defines the (exponentiated) effective action functional which is a section

$$
Z=\exp (-S): \mathcal{A} \longrightarrow \operatorname{det},
$$

with $S(A)=-\log \operatorname{det}\left(\not_{A}\right)$. The action of $\Gamma$ on $\mathcal{A}$ via gauge transformations can be lifted to the determinant line bundle, which hence descends to a line bundle over the moduli space of gauge connections $\mathcal{A} / \Gamma$.

Being an affine space, $\mathcal{A}$ is contractible, so over $\mathcal{A}$ we can trivialise the determinant line bundle and hence identify the effective action functional $Z$ with a complex function. However, this might not be possible over the orbit space $\mathcal{A} / \Gamma$ : if the descended line bundle is non-trivial then we cannot identify the effective action functional with a complex function in a gauge-invariant way, i.e. the gauge symmetry is anomalous. The line bundle over $\mathcal{A} / \Gamma$ is trivial if and only if we can choose a $\Gamma$-equivariant trivialisation of the line $Z(A)=\operatorname{det}\left(\not_{A}\right)$. By our general discussion above, the obstruction to this is an element of $\mathrm{H}^{1}\left(\Gamma ; \mathrm{U}(1)^{\mathcal{A}}\right)$, where $\mathrm{U}(1)^{\mathcal{A}}$ is the diffeological space of maps from $\mathcal{A}$ to $\mathrm{U}(1)$. An explicit formula for this smooth 1-cocycle is obtained in [CM95].

7.2. Odd dimensions: the Faddeev-Mickelsson-Shatashvili anomaly. We shall now generalise the construction from the Sect. 7.1 to bundle gerbes. For this, we need to introduce a categorification of the first group cohomology which takes values in a smooth abelian 2-group. We use a definition along the lines of [BMS19], adjusted to the smooth setting.

Definition 7.1. Let $G$ be a Lie group and A a smooth abelian 2-group equipped with a left action $\rho$ of $\underline{G}$. A smooth higher 1-cocycle on $G$ with values in A consists of

- a morphism $\lambda: \underline{G} \longrightarrow \mathrm{A}, g \longmapsto \lambda_{g}$, in $\mathcal{H}$, and 
- a smooth natural isomorphism $\chi_{g, g^{\prime}}: \lambda_{g} \otimes \rho_{g}\left(\lambda_{g^{\prime}}\right) \longrightarrow \lambda_{g g^{\prime}}$ of smooth functors $\underline{G} \times \operatorname{eart} \underline{G} \longrightarrow \mathrm{A}$,

such that for every $c \in$ Cart:

- $\lambda_{e_{c}}=I_{c}$ where $I_{c}$ is the monoidal identity object of the fibre $\mathrm{A}_{\mid c}$ of A over $c \in$ Cart, and where $e_{c}: c \longrightarrow G$ is the constant map at the identity element of $G$,

- $\chi_{e_{c}}$, and $\chi_{\cdot, e_{c}}$ agree with the left and right unitor morphisms in $\mathrm{A}_{\mid c}$, and

- the diagram

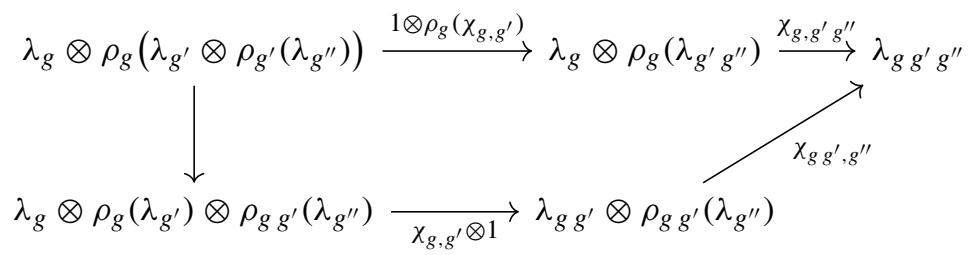

commutes for all $g, g^{\prime}, g^{\prime \prime} \in G(c)$.

We will also need the concept of a higher coboundary.

Definition 7.2. Let $(\lambda, \chi)$ and $\left(\lambda^{\prime}, \chi^{\prime}\right)$ be higher 1-cocycles on a Lie group $G$ valued in a smooth abelian 2-group $A$. A higher coboundary between $(\lambda, \chi)$ and $\left(\lambda^{\prime}, \chi^{\prime}\right)$ consists of

- a morphism $\theta: * \longrightarrow \mathrm{A}$, and

- smooth isomorphisms $\omega_{g}: \lambda_{g} \otimes \rho_{g}(\theta) \longrightarrow \theta \otimes \lambda_{g}^{\prime}$ for every $g \in \underline{G}$,

such that $\omega_{e_{c}}$ agrees with the symmetry isomorphism $\beta_{\mathrm{A}}$, and the diagram

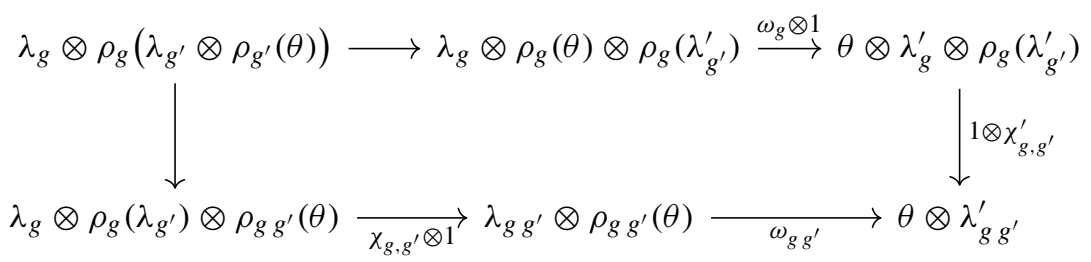

commutes for all $c \in$ Cart and $g, g^{\prime}, g^{\prime \prime} \in G(c)$.

Remark 7.3. There is a natural definition of morphisms between higher coboundaries, but these are not relevant for our purposes.

Let $G$ be a connected Lie group, $M$ a manifold with smooth $G$-action $\Phi: G \times M \longrightarrow$ $M$, and $(\mathcal{G}, A, \chi)$ a $G$-equivariant bundle gerbe on $M$. The equivariant structure on $\mathcal{G}$ can be described by a splitting $s_{\mathcal{G}}: \underline{G} \longrightarrow \operatorname{Sym}_{G}(\mathcal{G})$, as explained in Sect. 5.6. Assume furthermore that $\mathcal{G}$ is trivial as bundle gerbe, i.e. there exists a 1-isomorphism $E: \mathcal{I} \longrightarrow \mathcal{G}$. From the results in Sect. 5.6 we can deduce that the obstruction to the existence of an equivariant structure on $E$ is the higher 1-cocycle

$$
(f: c \longrightarrow G) \longmapsto s_{\mathcal{G}}(f)^{-1} \circ \widehat{E}\left(s_{\mathcal{I}}(f)\right) \in \operatorname{BGrb}(c \times M)\left(\operatorname{pr}_{M}^{*} \mathcal{G}, \operatorname{pr}_{M}^{*} \mathcal{G}\right) \cong \operatorname{HLBdl}(c \times M) .
$$

This 1-cocycle is trivial precisely if there exists a natural isomorphism to the constant 1 -cocycle at the trivial line bundle $I$. The choice of such an isomorphism corresponds to the equivariant structure on $E$. The isomorphisms $\chi$ in Definition 7.1 use the smooth 
2-group structure on $\mathrm{HLBdl}^{M}$, and the isomorphisms which are part of the splittings $s_{\mathcal{G}}$ and $s_{\mathcal{I}}$, and $\widehat{E}$. Generally, two 1-isomorphisms $\mathcal{G} \longrightarrow \mathcal{G}^{\prime}$ of bundle gerbes differ by the action of a 1-automorphism of $\mathcal{G}^{\prime}$. Hence the obstruction to the existence of an equivariant isomorphism is an element of the first higher group cohomology of $G$ with coefficients in the smooth abelian 2-group $\mathrm{HLBdl}^{M}$.

Let us now explain the relation to the Hamiltonian description of chiral anomalies in terms of bundle gerbes, which was worked out in [Mic85,CM95,CM96,CMM97, CMM00]. Let $M$ be a based odd-dimensional compact Riemannian spin manifold, ${ }^{9} P$ a principal $G$-bundle on $M$, and $\rho: G \longrightarrow \operatorname{End}(V)$ a representation of $G$ describing the matter content of the gauge theory. Again we denote by $\mathcal{A}$ the affine space of connections on $P$ and by $\Gamma$ the pointed group of gauge transformations. For every $A \in \mathcal{A}$ we can construct a massless Dirac operator

$$
\not D_{A}: \Gamma(M ; S \otimes V) \longrightarrow \Gamma(M ; S \otimes V)
$$

where $S \longrightarrow M$ is the spinor bundle. The Dirac operator is a first order self-adjoint elliptic differential operator, which serves as the first quantised Hamiltonian acting on the one-particle Hilbert space $\mathcal{H}=\Gamma(M ; S \otimes V)$.

To define the fermionic Fock space $\mathcal{F}_{A}(\mathcal{H})$ of the quantum field theory in the presence of a gauge field $A \in \mathcal{A}$, one has to pick a polarisation $\mathcal{H}=\mathcal{H}_{+}(A) \oplus \mathcal{H}_{-}(A)$. In general there are gauge fields $A \in \mathcal{A}$ for which the Dirac operator $D_{A}$ has zero modes; for these fields there is no universal and natural way of choosing such a polarisation. Denote by $\mathcal{A}_{0} \subset \mathcal{A} \times \mathbb{R}$ the subset of pairs $(A, r)$ such that the real number $r$ is not contained in the spectrum of $\not_{A}$. To equip this space with a diffeology we use the discrete diffeology on $\mathbb{R}$. For every point $(A, r) \in \mathcal{A}_{0}$ we get a decomposition of the one-particle Hilbert space

$$
\mathcal{H}=\mathcal{H}_{+}(A, r) \oplus \mathcal{H}_{-}(A, r)
$$

into the positive and negative eigenstates of the operator $\not_{A}-r 1_{\mathcal{H}}$. The corresponding Fock bundle $\mathcal{F}(\mathcal{H}) \longrightarrow \mathcal{A}_{0}$ has fibres

$$
\mathcal{F}(\mathcal{H})_{\mid(A, r)}=\wedge \mathcal{H}_{+}(A, r) \otimes \wedge \mathcal{H}_{-}(A, r)^{\vee}
$$

It is shown in [CM96] that the corresponding projective Hilbert bundle descends to a bundle over $\mathcal{A}$, and hence it induces a bundle gerbe $\mathcal{G}$ on $\mathcal{A}$. The bundle gerbe can be explicitly described in terms of determinant lines associated to families of Dirac operators, see [CM96, Section 5] for details. Since $\mathcal{A}$ is contractible, over $\mathcal{A}$ the projective Hilbert bundle is trivial and hence is associated to a bundle of Hilbert spaces. Again the action of $\Gamma$ on $\mathcal{A}$ lifts to an equivariant structure on $\mathcal{G}$. Therefore $\mathcal{G}$ as well as the projective Hilbert bundle descends to the orbit space $\mathcal{A} / \Gamma$. The Faddeev-Mickelsson-Shatashvili anomaly is the obstruction to the existence of a well-defined bundle of Hilbert spaces over $\mathcal{A} / \Gamma$, i.e. to the existence of a trivialisation of the descended projective Hilbert bundle. Equivalently, the anomaly vanishes if and only if $\mathcal{G}$ descends to a trivial bundle gerbe on $\mathcal{A} / \Gamma$. This in turn is the case if and only if $\mathcal{G}$ is trivial as a $\Gamma$-equivariant bundle gerbe on $\mathcal{A}$.

\footnotetext{
9 The type of Hamiltonian anomaly discussed here can only occur on odd-dimensional manifolds, since otherwise the chirality operator could be used to define consistent polarisations.
} 
From the general discussion above it follows that the obstruction to the equivariant triviality of $\mathcal{G}$ is a smooth higher 1 -cocycle on $\Gamma$ with values in $\operatorname{HLBdl}^{\mathcal{A}}$. Because $\mathcal{A}$ is contractible, there is an equivalence

$$
\operatorname{HLBdl}^{\mathcal{A}} \cong * / / \mathrm{U}(1)^{\mathcal{A}}
$$

with the smooth category with one object and the diffeological mapping space $U(1)^{\mathcal{A}}$ as morphisms. Since this is a smooth 2-group with one object, Definition 7.1 in this instance is equivalent to the definition of an ordinary group 2-cocycle on $\Gamma$ with values in $\mathrm{U}(1)^{\mathcal{A}}$. That the obstruction to the vanishing of the anomaly is a 2-cocycle of this form is well-known, see e.g. [CM96]; this cocycle reproduces the usual Schwinger terms in the commutator anomaly for the gauge group action. What is new here is the construction of a smooth higher 1-cocycle, which only reduces to an ordinary 2cocycle because the space $\mathcal{A}$ is contractible, as well as a rigorous incorporation of the smooth structures. Computing this cocycle explicitly and comparing it to the FaddeevMickelsson-Shatashvili cocycle is beyond the scope of this paper. We expect this to be possible using index theory following [CMM97].

\section{Application III: The String Group}

Any compact simple Lie group $G$ has homotopy groups $\pi_{3}(G) \cong \mathrm{H}^{3}(G ; \mathbb{Z}) \cong \mathbb{Z}$ and $\pi_{i}(G) \cong \mathrm{H}^{i}(G ; \mathbb{Z}) \cong 0$ for $i=0,1,2$; that is, $G$ is 2 -connected. It is of interest in topology and geometry (see e.g. [DHH11,Sto96,ST04]), as well as in string theory (see e.g. [SS20]), to study 3-connected approximations to $G$ that arise as group extensions of $G$. We denote such approximating objects by $\operatorname{String}(G)$ and call them models for the string group of $G$. There is a variety of interpretations of what this means, based on different choices of ambient higher categories in which one considers $G$ to be a group object. The general theme, however, is that one needs a way to realise a generator of $\pi_{3}(G) \cong \mathbb{Z}$ geometrically in the chosen framework, and a string group model for $G$ will generally be a choice of such a generator.

In this final section we recall the definition and construction of a topological string group model, and show that our extensions $\operatorname{Sym}_{G}(P)$ from Sect. 3 provide a smooth enhancement thereof. We then propose the smooth 2-groups $\operatorname{Sym}_{G}(\mathcal{G})$ and $\mathfrak{D e} \mathfrak{S}_{\mathrm{L}}$ from Sect. 5 as new string group models, for the specific choices of $M=G$ and of a gerbe $\mathcal{G}$ on $G$ whose Dixmier-Douady class generates $\mathrm{H}^{3}(G ; \mathbb{Z}) \cong \mathbb{Z}$. A model for $\operatorname{String}(G)$ which is very similar in spirit to our model $\operatorname{Sym}_{G}(\mathcal{G})$ was found in [FRS16]. However, that construction relies on the choice of connection on $\mathcal{G}$ and considers connection-preserving symmetries of $\mathcal{G}$. Here, in contrast, we exhibit a construction of string group models for $G$ from symmetries of gerbes on $G$ without connections, and thus from representatives of the third integer cohomology of $G$ rather than its differential cohomology. We defer further details and comments to Sect. 8.2.

8.1. A smooth string group model. The simplest and original framework for considering string group models is that of topological spaces.

Definition 8.1. Let $G$ be a compact simple simply-connected Lie group. A topological model for the string group $\operatorname{String}(G)$ of $G$ is a topological 3-connected group $\operatorname{String}_{\mathrm{t}}(G)$ along with a fibration $\operatorname{String}_{\mathrm{t}}(G) \longrightarrow G$ whose typical fibre is an Eilenberg-MacLane space $K(\mathbb{Z} ; 2)$. 
Using homotopy and cohomology groups one shows that $\operatorname{String}_{t}(G)$ cannot be a finite-dimen-sional Lie group [NSW13]. If a topological string group model can be enhanced to consist of smooth spaces (such as Fréchet manifolds or diffeological spaces), we denote it by $\operatorname{String}(G)$ and refer to it as a smooth model for the string group of $G$.

We recall Stolz' model as a topological group [Sto96]: let PU denote the projective unitary group of an infinite-dimensional separable Hilbert space. As a consequence of Kuiper's Theorem, $\mathbb{P U}$ has homotopy type $K(\mathbb{Z} ; 2)$. Hence the classifying space BPU has homotopy type $K(\mathbb{Z} ; 3)$, while at the same time it classifies topological principal $\mathbb{P} U$-bundles. In particular, isomorphism classes of $\mathbb{P U}$-bundles on a space $X$ are in one-to-one correspondence with elements of the set $\mathrm{H}^{3}(X ; \mathbb{Z})$.

Let $P \longrightarrow G$ be a principal $\mathbb{P U}$-bundle on $G$ such that $P$ corresponds to a generator of $\mathrm{H}^{3}(G ; \mathbb{Z}) \cong \mathbb{Z}$; such $\mathbb{P U}$-bundles on $G$ are called basic. Let $\widehat{G}$ denote the group of PU-equivariant homeomorphisms of $P$ to itself which act on $G$ as left multiplication by some element of $G$. We can topologise $\widehat{G}$ as a subgroup of the topological group of homeomorphisms $P \longrightarrow P$. Thus $\widehat{G}$ comes with a continuous surjective group homomorphism $\widehat{G} \longrightarrow G$. The gauge group $\operatorname{Gau}(P)$ is the subgroup of $\widehat{G}$ of those elements whose projection to $G$ is the identity element $e \in G$.

Theorem 8.2 [[Sto96, Section 5] and [NSW13]]. The extension of topological groups

$$
1 \longrightarrow \operatorname{Gau}(P) \longrightarrow \widehat{G} \longrightarrow G \longrightarrow 1
$$

exhibits $\widehat{G}$ as a topological model for $\operatorname{String}(G)$.

The crux of the proof of this theorem is showing that $\mathrm{Gau}(P)$ is homotopy equivalent to $\mathbb{P U}$, i.e. that it is an Eilenberg-MacLane space $K(\mathbb{Z} ; 2)$. Part of the content in [NSW13] is to enhance this topological string group model to a smooth model in the sense that the groups appearing are Fréchet Lie groups.

The group extension $\widehat{G} \longrightarrow G$ agrees with the extension $\operatorname{Sym}_{G}(P) \longrightarrow G$ constructed in Sect. 3 when we set $M=G$ and $H=\mathbb{P U}$, and let $G$ act on itself by left multiplication. Thus we immediately obtain

Corollary 8.3. Let $P \longrightarrow G$ be a basic PU-bundle. The extension of diffeological groups

$$
1 \longrightarrow \operatorname{Gau}(P) \longrightarrow \operatorname{Sym}_{G}(P) \longrightarrow G \longrightarrow 1
$$

exhibits $\operatorname{Sym}_{G}(P)$ as a smooth model for $\operatorname{String}(G)$.

8.2. Smooth string 2-group models. Let $G$ be a compact simply-connected Lie group, and let $\mathcal{G}$ be a bundle gerbe on $G$ whose Dixmier-Douady class generates $\mathrm{H}^{3}(G ; \mathbb{Z})$; such a bundle gerbe is said to be basic. Let $\Phi: G \times G \longrightarrow G$ denote the left action of $G$ on itself by left multiplication. In the spirit of Sect. 5, it is reasonable to expect that we should also be able to interpret the smooth 2-groups $\operatorname{Sym}_{G}(\mathcal{G})$ and $\mathfrak{D e s} \mathrm{L}$ as models for $\operatorname{String}(G)$. The idea of constructing $\operatorname{String}(G)$ as a smooth 2-group has also been considered in e.g. [BCSS07,SP11, Wal12a, NSW13, FRS16]. In the remainder of this section we will describe how $\operatorname{Sym}_{G}(\mathcal{G})$ can be seen as a string 2-group model. By Theorem 5.36 it then follows that $\mathfrak{D} \mathfrak{e} \mathfrak{S}_{\mathrm{L}}$ is also a model for $\operatorname{String}(G)$.

Smooth string 2-group models usually consist of extensions of $G$ by the smooth 2-group BU(1), the delooping of the smooth abelian group $U(1)$. However, recall that 
in Theorem 5.27 we established $\operatorname{Sym}_{G}(\mathcal{G})$ as an extension of $\underline{G}$ by the smooth abelian 2-group $\mathrm{HLBdl}^{G}$. Our point here is that what matters for string group models is only the homotopy type of the fibre and the total space of the map $\operatorname{String}(G) \longrightarrow G$, so that there is a lot of flexibility in choosing the smooth 2-group A that extends $G$. Observe that this ambiguity is inherent already in Definition 8.1. This forces us to state which smooth 2groups $\mathrm{A}$ are admissible in order to obtain smooth 2-group extensions of $G$ that deserve to be called string group models. Our proposed definition for smooth string 2-group models emphasises the structure of $A$ as a smooth analogue of an Eilenberg-MacLane space $K(\mathbb{Z} ; 2)$. Note that for every smooth abelian 2-group A and any manifold $M$, we can define Čech cohomology of $M$ with coefficients in A by evaluating (a delooping of A) on the Čech nerve of good open coverings of $M$.

The definition of a smooth string 2-group model is thus a two-step process: we first fix the homotopy type of the extending 2-group $A$ in a geometric way, and then we have to make precise when an A-extension of $G$ has the correct homotopy type.

Definition 8.4. Let $H$ be a diffeological group. The delooping $\mathrm{B} \underline{H} \in \mathcal{H}$ is the category fibred in groupoids over Cart whose objects are the Cartesian spaces $c \in$ Cart, and whose morphisms $c_{0} \longrightarrow c_{1}$ are pairs $\left(f_{01}, h_{01}\right)$ of smooth maps $f_{01}: c_{0} \longrightarrow c_{1}$ and $h_{01}: c_{0} \longrightarrow H$. Composition of morphisms is given by $\left(f_{12}, h_{12}\right) \circ\left(f_{01}, h_{01}\right)=$ $\left(f_{12} \circ f_{01}, h_{01}\left(h_{12} \circ f_{01}\right)\right)$.

If $H$ is abelian, then $\mathrm{B} \underline{H}$ naturally becomes a smooth abelian 2-group.

Definition 8.5. A smooth 2-group $A$ is string-admissible if it is abelian and equivalent (as a smooth 2-group) to the delooping $\mathrm{B} \underline{H}$ of a diffeological abelian group $H$ whose underlying topological space is an Eilenberg-MacLane space $K(\mathbb{Z} ; 2)$.

From the equivalence $\mathrm{A} \simeq \mathrm{B} \underline{H}$ it follows that Čech cohomology with coefficients in $\mathrm{A}$ is equivalent to Čech cohomology with coefficients in $H$, shifted by one degree. Then since $H$ has homotopy type $K(\mathbb{Z} ; 2)$, it follows that there are isomorphisms

$$
\check{\mathrm{H}}^{k}(M ; \mathrm{A}) \cong \check{\mathrm{H}}^{k}(M ; \mathrm{B} \underline{H}) \cong\left[M, \mathrm{~B}^{k+1} H\right] \cong[M, K(\mathbb{Z} ; k+2)] \cong \mathrm{H}^{k+2}(M ; \mathbb{Z})
$$

for all $k \in \mathbb{N}$. (For the notion of Čech cohomology with coefficients in higher smooth groups, we refer the reader to [Sch13,NSS15].)

From any smooth principal 2-bundle $\mathrm{P} \longrightarrow \underline{M}$ over a manifold $M$ with structure 2-group A, we can distil a Čech cohomology class as follows: let $\mathcal{U}=\left\{U_{i}\right\}_{i \in I}$ be a good open covering of $M$. Denote intersections by $U_{i_{1} \cdots i_{n}}:=U_{i_{1}} \cap \cdots \cap U_{i_{n}}$. Viewing the (intersections of) open patches $U_{i_{1} \cdots i_{n}} \hookrightarrow M$ as objects in $\underline{M}$, we denote by $\mathrm{P}_{\mid U_{i_{1} \cdots i_{n}}}$ the fibres of $\mathrm{P}$ over these objects. By Definition 5.17, we can choose an object $\psi_{i} \in \mathrm{P}_{\mid U_{i}}$ for every $i \in I$. We can further choose an object $\mathrm{a}_{i j} \in \mathrm{A}_{\mid U_{i j}}$ for every $i, j \in I$ and an isomorphism $g_{i j}: \psi_{i \mid U_{i j}} \otimes \mathrm{a}_{i j} \longrightarrow \psi_{j \mid U_{i j}}$ in $\mathrm{P}_{\mid U_{i j}}$ (where we have chosen pullbacks of $\psi_{i}$ and $\psi_{j}$ to $\left.\mathrm{P}_{\mid U_{i j}}\right)$. Over the triple overlaps $U_{i j k}$ we obtain isomorphisms

$$
\beta_{i j k}: \psi_{i \mid U_{i j k}} \otimes \mathrm{a}_{i j \mid U_{i j k}} \otimes \mathrm{a}_{j k \mid U_{i j k}} \longrightarrow \psi_{i \mid U_{i j k}} \otimes \mathrm{a}_{i k \mid U_{i j k}},
$$

which are uniquely determined by the properties of the Grothendieck fibration $\mathrm{P} \longrightarrow \underline{M}$ (as previously, since $\underline{M}$ has discrete fibres, it follows that $\mathrm{P} \times{ }_{\underline{M}}^{\mathrm{h}} \mathrm{P}=\mathrm{P} \times{ }_{\underline{M}} \mathrm{P}$ ). Since the morphisms $\left(1_{\psi_{i \mid U_{i j k}}}, \beta_{i j k}\right)$ lie in the image of the action functor $\mathrm{P} \times \mathrm{A} \longrightarrow \mathrm{P} \times{ }_{\underline{M}} \mathrm{P}$, there are unique isomorphisms

$$
\alpha_{i j k}: \mathrm{a}_{i j \mid U_{i j k}} \otimes_{\mathrm{A}} \mathrm{a}_{j k \mid U_{i j k}} \longrightarrow \mathrm{a}_{i k \mid U_{i j k}}
$$


in $\mathrm{A}_{\mid U_{i j k}}$, which satisfy the required coherence condition over quadruple overlaps by the fact that they are constructed as Cartesian lifts of identity morphisms. Hence these data assemble into an A-valued Čech 1-cocycle on $M$ with respect to the open covering $\mathcal{U}$. One can check that other choices of coverings and sections lead to 1-cocycles that become equivalent to $\left(\mathrm{a}_{i j}, \alpha_{i j k}\right)$ when passing to a common refinement of good open coverings.

Definition 8.6. Let $G$ be a compact simply-connected Lie group, and let A be a stringadmissible smooth 2-group. A smooth 2-group model for $\operatorname{String}(G)$ is a smooth 2-group extension

$$
1 \longrightarrow \mathrm{A} \longrightarrow \operatorname{String}(G) \longrightarrow \underline{G} \longrightarrow 1
$$

such that the principal 2-bundle $\operatorname{String}(G) \longrightarrow G$ represents a generator of $\mathrm{H}^{3}(G ; \mathbb{Z}) \cong$ $\mathbb{Z}$ under the isomorphism $\check{\mathrm{H}}^{1}(G ; \mathrm{A}) \cong \mathrm{H}^{3}(G ; \underline{\mathbb{Z}})$.

With these definitions we have

Theorem 8.7. For any 2-connected manifold $M$, the smooth 2-group $\mathrm{HLBdl}^{M}$ is stringadmissible.

Theorem 8.8. Let $G$ be a compact simply-connected Lie group, and let $\mathcal{G} \in \operatorname{BGrb}(G)$ be a basic bundle gerbe. Let $\operatorname{Sym}_{G}(\mathcal{G})$ and $\mathfrak{D e s L}$ be the smooth 2-group extensions of $\underline{G}$ by $\mathrm{HLBdl}^{G}$ constructed from $\mathcal{G}$ with respect to the left action of $G$ on itself by left multiplication. Then both $\operatorname{Sym}_{G}(\mathcal{G})$ and $\mathfrak{D e s} \mathrm{L}$ are smooth 2-group models for $\operatorname{String}(G)$ in the sense of Definition 8.6.

Remark 8.9. Note that there is a model for $\operatorname{String}(G)$ based on regression and transgression of the basic gerbe on $G$ [Wal12a]. Similarly, our model for $\operatorname{String}(G)$ which we obtain from $\mathfrak{D e s}$ also heavily relies on the transgression-regression formalism. However, the resulting models are very different: Waldorf's model in [Wal12a] is the regression of the transgression of the basic gerbe. Waldorf observes that this gerbe picks up a multiplicative structure upon application of $\mathcal{R} \circ \mathcal{T}$. Since any gerbe on a manifold can be seen as an extension of $M$ by BU(1) as Lie groupoids, the resulting gerbe provides a string group model in the sense of [SP11]. Our construction differs from this significantly not only in the fact that we work with smooth 2-groups instead of Lie 2-groups; most notably, we obtain an extension of $G$ by the much larger smooth 2-group $\mathrm{B}\left(\mathrm{U}(1)^{H}\right)$, which is not equivalent to $\mathrm{BU}(1)$ as a smooth 2-group, but only on the level of their underlying homotopy types (see Theorem 8.7 as well as [Bun20b]).

The rest of this section is devoted to the proofs of Theorems 8.7 and 8.8. We begin with a few results that will combine to prove that $\mathrm{HLBdl}^{M}$ is string-admissible. Then we will prove Theorem 8.8 by observing that the $\mathrm{HLBdl}^{G}$-valued Cech 1-cocycles we obtain from the 2-bundle $\operatorname{Sym}_{G}(\mathcal{G})$ agree with those obtained from local trivialisations of the bundle gerbe $\mathcal{G}$.

Lemma 8.10. Let $M$ be a simply-connected manifold and $x \in M$ a fixed base point. Then the evaluation map $\mathrm{ev}_{x}: \mathrm{U}(1)^{M} \longrightarrow \mathrm{U}(1), g \longmapsto g(x)$ and the inclusion $\mathrm{C}: \mathrm{U}(1) \longrightarrow$ $\mathrm{U}(1)^{M}$ as constant maps form a homotopy equivalence of diffeological spaces. ${ }^{10}$

${ }^{10}$ See Example 2.6 for the definition of the diffeological mapping space. 
Proof. Since $\mathrm{ev}_{x} \circ \mathrm{C}=1_{\mathrm{U}(1)}$, it is enough to construct a smooth homotopy $\mathrm{C} \circ \mathrm{ev}_{x} \cong$ $1_{\mathrm{U}(1)^{M}}$. Let $\pi: \mathbb{R} \longrightarrow \mathrm{U}(1), r \longmapsto \exp (2 \pi \mathrm{i} r)$ be the universal cover of $\mathrm{U}(1)$. The assumption that $M$ is simply-connected implies (see e.g. [Bre93, Theorem 4.1]) that the diagram

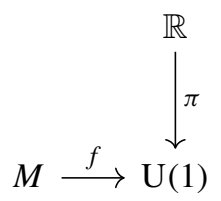

admits a unique continuous lift $\widehat{f}: M \longrightarrow \mathbb{R}$ for arbitrary $f \in \mathrm{U}(1)^{M}$ after fixing the lift at one point. We verify that the map $\widehat{f}$ is smooth. Fix a point $y \in M$ and a sufficiently small open neighbourhood $U_{y}$ of $y$ which we identify with an open subset of $\mathbb{R}^{d}$, where $d=\operatorname{dim}(M)$. The restriction of $f$ to $U_{y}$ is a plot of $\mathrm{U}(1)$. Hence by Proposition 2.9 it admits a smooth lift $\widehat{f_{y}}: U_{y} \longrightarrow \mathbb{R}$ for sufficiently small $U_{y}$. From the uniqueness statement for lifts we obtain $\widehat{f}_{\mid U_{y}}=\widehat{f}_{y}+r_{y}$ for a fixed integer $r_{y} \in \mathbb{Z}$. This implies that $\widehat{f}$ is smooth and hence shows that the map $\pi^{M}: \mathbb{R}^{M} \longrightarrow \mathrm{U}(1)^{M}$ is surjective.

Consider the commutative diagram

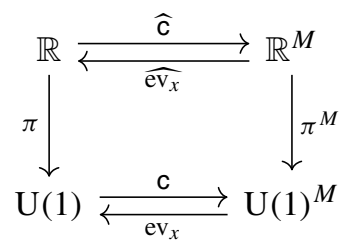

where $\widehat{\mathrm{c}}(r)(y)=r$ for all $y \in M$, and where $\widehat{\mathrm{ev}}_{x}\left(g^{\prime}\right)=g^{\prime}(x)$ for all $g^{\prime} \in \mathbb{R}^{M}$. We define a homotopy $\widehat{h}: 1_{\mathbb{R}^{M}} \longrightarrow \widehat{\mathrm{C}} \circ \widehat{\mathrm{ev}}_{x}$ by setting $\widehat{h}_{t}\left(g^{\prime}\right)(y)=g^{\prime}(y)(1-t)+g^{\prime}(x) t$. This homotopy descends to the desired homotopy $h: 1_{\mathrm{U}(1)^{M}} \longrightarrow \mathrm{C} \circ \mathrm{ev}_{x}$.

We verify that the homotopy $h$ is smooth: let $n$ be a natural number, $c \cong \mathbb{R}^{n}$ a Cartesian space and $f: c \longrightarrow \mathrm{U}(1)^{M} \times[0,1]$ a plot; that is, the maps $\operatorname{pr}_{[0,1]} \circ f: c \longrightarrow[0,1]$ and

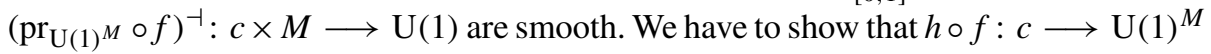
is a plot. By the arguments above we can lift $\left(\operatorname{pr}_{\mathrm{U}(1)^{M}} \circ f\right)^{-1}$ to a smooth map $c \times M \longrightarrow \mathbb{R}$ because $c \times M$ is simply-connected. This implies that we can lift $f$ to a smooth map $\widehat{f}: c \longrightarrow \mathbb{R}^{M} \times[0,1]$ making the diagram

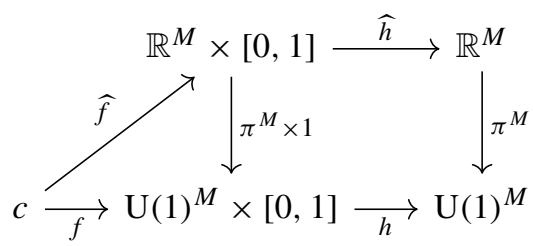

commute. The result now follows since $\widehat{h}$ and the projection $\pi^{M}: \mathbb{R}^{M} \longrightarrow \mathrm{U}(1)^{M}$ are smooth.

Lemma 8.11. There is an inclusion $\mathrm{B}\left(\mathrm{U}(1)^{M}\right) \hookrightarrow \mathrm{HLBdl}^{M}$ of smooth 2-groups which is given by sending $c \in \mathrm{B}\left(\mathrm{U}(1)^{M}\right)$ (cf. Definition 8.4 ) to the trivial line bundle on $c \times M$. If $\mathrm{H}^{2}(M ; \mathbb{Z})=0$, then this inclusion is an equivalence. 
Proof. We readily see that the inclusion respects the group structures, and that it is fully faithful. If $\mathrm{H}^{2}(M ; \mathbb{Z})=0$, then $\operatorname{HLBdl}^{M}{ }_{\mid c} \cong \operatorname{HLBdl}(c \times M)$ is connected, so that in this case the inclusion is also fully faithful on all fibres. Thus it is an equivalence on every fibre and hence an equivalence in the 2-category $\mathcal{H}$ by [Vis05, Proposition 3.36].

Combining Lemmas 8.10 and 8.11 , we conclude that $\mathrm{HLBdl}^{M}$ is string-admissible for any 2-connected manifold $M$; that is, we have proven Theorem 8.7.

For the diffeological group $H=\mathrm{U}(1)^{M}$ and a 2-connected manifold $M$, there is an explicit isomorphism $\check{\mathrm{H}}^{k}\left(X ; \mathrm{U}(1)^{M}\right) \cong \check{\mathrm{H}}^{k}(X ; \mathrm{U}(1))$ for $k>0$, for any manifold $X$, given by

Proposition 8.12. Let $M$ be a 2-connected manifold with a fixed base point $x \in M$. For any manifold $X$, evaluation at $x \in M$ induces an isomorphism $\check{\mathrm{H}}^{k}\left(X ; \mathrm{U}(1)^{M}\right) \cong$ $\check{\mathrm{H}}^{k}(X ; \mathrm{U}(1))$ for $k>0$ of $\check{C}$ ech cohomology groups with coefficients in the sheaves of smooth $\mathrm{U}(1)^{M}$-valued and $\mathrm{U}(1)$-valued functions, respectively.

Proof. Consider the sequence of diffeological groups

$$
1 \longrightarrow \mathbb{Z} \longrightarrow \mathbb{R}^{M} \longrightarrow \mathrm{U}(1)^{M} \longrightarrow 1
$$

which is exact by the argument from the proof of Lemma 8.10. The sheaf $\mathbb{R}^{M}$ admits a partition of unity by picking a partition of unity for the sheaf of smooth $\mathbb{R}$-valued functions and a constant extension to $\mathbb{R}^{M}$-valued functions; hence $\check{\mathrm{H}}^{k}\left(X ; \mathbb{R}^{M}\right)=0$ for any manifold $X$ and for any $k \geq 1$. Now the statement follows from applying the five lemma to the diagram

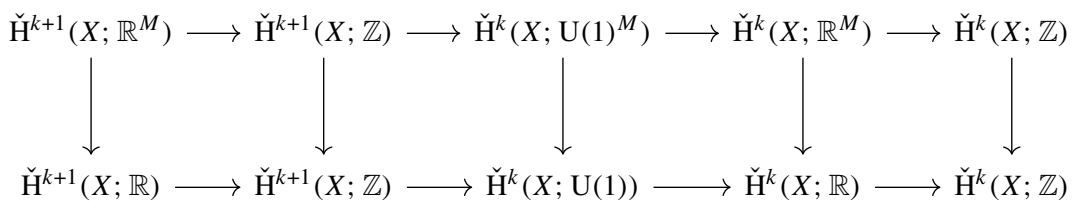

induced by the long exact sequence in sheaf cohomology and the evaluation at $x \in M$.

It remains to determine the $\check{C}$ ech cohomology class in $\check{\mathrm{H}}^{1}\left(G ; \mathrm{HLBdl}^{G}\right) \cong \mathrm{H}^{3}(G ; \mathbb{Z}) \cong$ $\mathbb{Z}$ determined by the extension $\operatorname{Sym}_{G}(\mathcal{G}) \longrightarrow \underline{G}$. The isomorphisms from Lemma 8.11 and Proposition 8.12 are useful in achieving this. From the smooth 2-group extension $\operatorname{Sym}_{G}(\mathcal{G}) \longrightarrow G$ we can extract a Čech 2 -cocycle on $G$ with values in the sheaf of smooth $\mathrm{U}(1)^{G}$-valued functions. To construct it, we first follow the procedure of the paragraph preceding Definition 8.6 to extract $\mathrm{HLBdl}^{G}$-valued cocycle data and then choose local trivialisations of the line bundles which comprise it (which amounts to choosing an inverse for the equivalence from Lemma 8.11).

Let $\mathcal{U}=\left\{U_{i}\right\}_{i \in I}$ be a good open cover of $G$, let $\pi_{i}: U_{i} \times G \longrightarrow G$ denote the projection onto the second factor, and let $m_{i}: U_{i} \times G \longrightarrow G$ be the multiplication map restricted to $U_{i} \times G$. We choose and fix 1-isomorphisms $\psi_{i}: m_{i}^{*} \mathcal{G} \longrightarrow \pi_{i}^{*} \mathcal{G}$ along with adjoint inverses $\psi_{i}^{-1}$, which induce equivalences $\operatorname{Sym}_{G}(\mathcal{G})_{\mid U_{i}} \cong \operatorname{HLBdl}\left(U_{i} \times G\right)$ of groupoids.

On double intersections $U_{i j}$ we can form the automorphism $\psi_{i j}:=\psi_{j \mid U_{i j}}^{-1} \circ \psi_{i \mid U_{i j}}$ of $m_{i j}^{*} \mathcal{G}$. The isomorphism $\psi_{i j}$ can be identified with a line bundle $L_{i j}$ on $U_{i j}^{i j} \times G$. Since $\mathrm{H}^{2}(G ; \mathbb{Z})=0$, we can choose and fix a trivialisation of $L_{i j}$ for each $i, j \in I$. 
On triple intersections $U_{i j k}$ we get a 2-isomorphism $\psi_{i j k}: \psi_{j k \mid U_{i j k}} \circ \psi_{i j \mid U_{i j k}} \longrightarrow$ $\psi_{i k \mid U_{i j k}}$ inducing an isomorphism $L_{j k} \otimes L_{i j} \longrightarrow L_{i k}$ of line bundles over $U_{i j k} \times G$. (In contrast to the construction in the paragraph above Definition 8.6, here the isomorphisms $\psi_{i j k}$ can be obtained directly from the choice of $\psi_{i j}$.) Using the trivialisations of these line bundles we obtain a smooth map $U_{i j k} \times G \longrightarrow \mathrm{U}(1)$ or equivalently a map $c_{i j k}: U_{i j k} \longrightarrow \mathrm{U}(1)^{G}$. The collection $c_{i j k}$ form a smooth $\mathrm{U}(1)^{G}$-valued Čech 2-cocycle.

The corresponding cohomology class is independent of all choices involved: let $\psi_{i}^{\prime}: m_{i}^{*} \mathcal{G} \longrightarrow \pi_{i}^{*} \mathcal{G}$ be a different set of 1 -isomorphisms. The automorphism $\psi_{i}^{-1} \circ \psi_{i}^{\prime}$ of $m_{i}^{*} \mathcal{G}$ can be identified with a line bundle $\Lambda_{i}$ over $U_{i} \times G$. The definition of $L_{i j}$ implies $L_{i j} \otimes \Lambda_{i} \cong \Lambda_{j} \otimes L_{i j}^{\prime}$. We can pick once and for all trivialisations of all bundles involved to identify this morphism with a function $A_{i j}: U_{i j} \times G \longrightarrow \mathrm{U}(1)$. The diagram

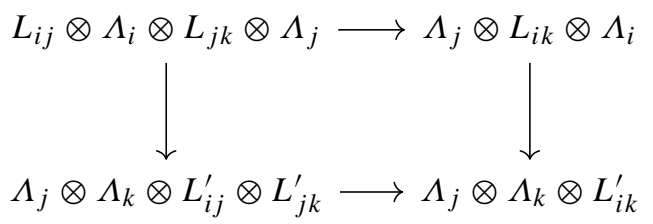

commutes over $U_{i j k}$, which follows from the fact that all inverses were chosen to be adjoint so that the corresponding diagram involving $\psi_{i}$ and $\psi_{i}^{\prime}$ commutes. Applying the trivialisations we get

$$
c_{i j k} A_{i k}=c_{i j k}^{\prime} A_{i j} A_{j k} .
$$

This argument also shows that the cocycles define the same cohomology class if $\psi_{i}^{\prime}=\psi_{i}$ and only the trivialisations of $L_{i j}$ differ.

The image of $c_{i j k}$ in $\check{\mathrm{H}}^{2}\left(G ; \mathrm{U}(1)^{G}\right)$ under the isomorphism $\check{\mathrm{H}}^{2}\left(G ; \mathrm{U}(1){ }^{G}\right) \cong \check{\mathrm{H}}^{2}$ $(G ; \mathrm{U}(1))$ of Proposition 8.12 can be computed by restricting each $\psi_{i}$ to $U_{i} \times\{e\} \subset$ $U_{i} \times G$; the restriction $\psi_{\mid U_{i} \times\{e\}}$ is a 1-isomorphism $\left.\mathcal{G}_{\mid U_{i}} \longrightarrow \mathcal{G}\right|_{e}$. After fixing once and for all a trivialisation of $\mathcal{G}_{\mid e}$, this is just a trivialisation of $\mathcal{G}_{\mid U_{i}}$. This shows that the image of $c_{i j k}$ in $\check{\mathrm{H}}^{2}(G ; \mathrm{U}(1)) \cong \mathrm{H}^{3}(G ; \mathbb{Z})$ agrees with the cocycle $c_{\mathcal{G}}$ classifying the bundle gerbe $\mathcal{G}$, which proves Theorem 8.8.

Remark 8.13. The arguments involving cocycles can be adjusted to the simpler case of principal bundles over the Lie group $G$. In that case, starting from a principal U(1)-bundle $P$ on $G$ we get a principal U(1) ${ }^{G}$-bundle $\operatorname{Sym}_{G}(P)$ on $G$ which is homotopy equivalent to $P$. The homotopy equivalence is induced by the maps $\mathrm{ev}_{x}$ and C from Lemma 8.10. We can iterate the procedure to get larger and larger groups $\operatorname{Sym}_{G}\left(\cdots \operatorname{Sym}_{G}\left(\operatorname{Sym}_{G}(P)\right) \cdots\right)$. However, these groups are all topologically equivalent, so that iterating the procedure does not produce anything that is topologically novel.

Acknowledgements. We thank Jouko Mickelsson and Birgit Richter for helpful discussions and correspondence. This work was supported by the COST Action MP1405 "Quantum Structure of Spacetime", funded by the European Cooperation in Science and Technology (COST). The work of S.B. was partially supported by the RTG 1670 "Mathematics Inspired by String Theory and Quantum Field Theory". The work of L.M. was supported by the Doctoral Training Grant ST/N509099/1 from the UK Science and Technology Facilities Council (STFC). The work of R.J.S. was supported in part by the STFC Consolidated Grant ST/P000363/1 "Particle Theory at the Higgs Centre".

Funding Open Access funding enabled and organized by Projekt DEAL. 
Open Access This article is licensed under a Creative Commons Attribution 4.0 International License, which permits use, sharing, adaptation, distribution and reproduction in any medium or format, as long as you give appropriate credit to the original author(s) and the source, provide a link to the Creative Commons licence, and indicate if changes were made. The images or other third party material in this article are included in the article's Creative Commons licence, unless indicated otherwise in a credit line to the material. If material is not included in the article's Creative Commons licence and your intended use is not permitted by statutory regulation or exceeds the permitted use, you will need to obtain permission directly from the copyright holder. To view a copy of this licence, visit http://creativecommons.org/licenses/by/4.0/.

Publisher's Note Springer Nature remains neutral with regard to jurisdictional claims in published maps and institutional affiliations.

\section{A. Properties of Smooth Principal 2-Bundles}

A.1. Surjectivity on objects and homotopy pullbacks. Here we provide some technical background on smooth groupoids, as introduced in Definition 5.4.

Lemma A.1. Let $\pi: \mathrm{X} \longrightarrow$ Cart and $\pi^{\prime}: \mathrm{P} \longrightarrow$ Cart be objects in $\mathcal{H}$.

(1) Either $\mathrm{X}=\varnothing$ or $\pi$ is surjective on objects.

(2) Let $p: \mathrm{P} \longrightarrow \mathrm{X}$ be a morphism in $\mathcal{H}$ whose underlying functor is an essentially surjective Grothendieck fibration. Then $p$ is surjective on objects.

Proof. To see (1), observe that Cart has a terminal object $* \in$ Cart. Thus since $\pi$ is a Grothendieck fibration, if $\mathrm{X}_{\mid *}=\pi^{-1}(*)$ is non-empty then so is $\mathrm{X}_{\mid c}$ for any $c \in$ Cart. For any $c \in$ Cart there exists a morphism $x: * \longrightarrow c$ in Cart given by choosing any point $x \in c$. It follows that as soon as $\mathrm{X} \neq \varnothing$, it has only non-empty fibres over Cart.

Claim (2) follows from the general observation that a Grothendieck fibration is essentially surjective if and only if it is surjective on objects.

We now consider the setup of Definition 5.16.

Lemma A.2. Let $\mathcal{C}$ be a category, let $\pi_{i}: \mathrm{D}_{i} \longrightarrow \mathcal{C}$, for $i=0,1$, and $\pi_{\mathrm{E}}: \mathrm{E} \longrightarrow \mathcal{C}$ be Grothendieck fibrations in groupoids, and let $F_{i}: \mathrm{D}_{i} \longrightarrow \mathrm{E}$, for $i=0,1$, be morphisms of categories fibred in groupoids over $\mathrm{C}$.

(1) $\left(\mathrm{D}_{0} \times \mathrm{E}_{\mathrm{E}}^{\mathrm{h}} \mathrm{D}_{1}, \pi_{\mathrm{h}}\right) \in \mathcal{H}$, i.e. $\pi_{\mathrm{h}}$ is a Grothendieck fibration in groupoids.

(2) Any morphism $G=\left(G_{0}, G_{\mathrm{E}}, G_{1}\right)$ of diagrams

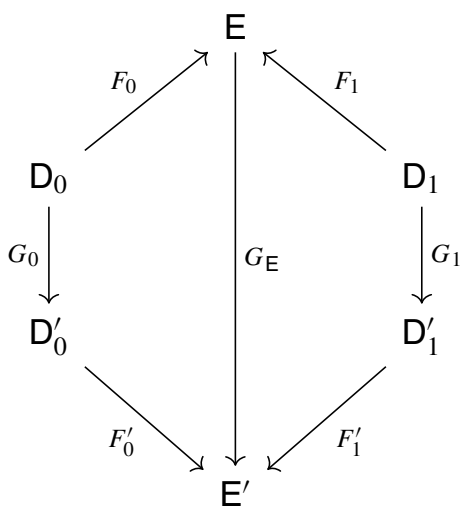

in $\mathcal{H}$, where all vertical morphisms are equivalences, induces an equivalence $D_{0} \times{ }_{E}^{h}$ $\mathrm{D}_{1} \longrightarrow \mathrm{D}_{0}^{\prime} \times{ }_{\mathrm{E}^{\prime}}^{\mathrm{h}} \mathrm{D}_{1}^{\prime}$. 
(3) If $F_{1}$ (resp. $F_{0}$ ) is a Grothendieck fibration in groupoids, then the inclusion $\mathrm{D}_{0} \times_{\mathrm{E}}$ $\mathrm{D}_{1} \hookrightarrow \mathrm{D}_{0} \times \mathrm{E}_{\mathrm{E}}^{\mathrm{h}} \mathrm{D}_{1}$ is an equivalence, and the projections $\mathrm{pr}_{0}^{\mathrm{h}}: \mathrm{D}_{0} \times \mathrm{E}_{\mathrm{E}}^{\mathrm{h}} \mathrm{D}_{1} \longrightarrow \mathrm{D}_{0}$ and $\mathrm{pr}_{0}: \mathrm{D}_{0} \times_{\mathrm{E}} \mathrm{D}_{1} \longrightarrow \mathrm{D}_{0}$ (resp. the projections $\mathrm{pr}_{1}^{\mathrm{h}}$ and $\mathrm{pr}_{1}$ to $\mathrm{D}_{1}$ ) are Grothendieck fibrations in groupoids.

Proof. To prove (1), we first show that every morphism in $\mathrm{D}_{0} \times{ }_{\mathrm{E}}^{\mathrm{h}} \mathrm{D}_{1}$ is $\pi_{\mathrm{h}}$-Cartesian. Consider morphisms $\left(\psi_{0}, \psi_{1}\right):\left(d_{0}^{\prime}, \eta^{\prime}, d_{1}^{\prime}\right) \longrightarrow\left(d_{0}^{\prime \prime}, \eta^{\prime \prime}, d_{1}^{\prime \prime}\right)$ and $\left(\varphi_{0}, \varphi_{1}\right):\left(d_{0}, \eta, d_{1}\right) \longrightarrow$ $\left(d_{0}^{\prime \prime}, \eta^{\prime \prime}, d_{1}^{\prime \prime}\right)$ in $\mathrm{D}_{0} \times \mathrm{E}_{\mathrm{E}}^{\mathrm{h}} \mathrm{D}_{1}$. By assumption $\pi_{0}\left(d_{0}\right)=\pi_{1}\left(d_{1}\right)$ in $\mathcal{C}$ (and analogously for $\left.d_{i}^{\prime}, d_{i}^{\prime \prime}\right)$, and $\pi_{0}\left(\psi_{0}\right)=\pi_{1}\left(\psi_{1}\right)$ (and analogously for $\left.\varphi_{i}\right)$. Let $\chi: \pi_{0}\left(d_{0}\right) \longrightarrow \pi_{0}\left(d_{0}^{\prime}\right)$ be a morphism in $\mathcal{C}$ such that $\pi_{0}\left(\psi_{0}\right) \circ \chi=\pi_{0}\left(\varphi_{0}\right)$. Since $\pi_{\mathrm{E}}$ is a Grothendieck fibration in groupoids, there exists a unique lift $\chi_{\mathrm{E}, i}: F_{i}\left(d_{i}\right) \longrightarrow F_{i}\left(d_{i}^{\prime}\right)$ induced by each of the pairs $\left(F_{i}\left(\psi_{i}\right), F_{i}\left(\varphi_{i}\right)\right)$ of morphisms in E. Similarly, the pairs $\left(\psi_{i}, \varphi_{i}\right)$ induce unique lifts $\chi_{i}: d_{i} \longrightarrow d_{i}^{\prime}$ of $\chi$ to $D_{i}$ along $\pi_{i}$. The uniqueness of lifts (along $\pi_{\mathrm{E}}$ ) implies that $F_{i}\left(\chi_{i}\right)=\chi_{\mathrm{E}, i}$ for $i=0,1$. It remains to show that $\left(\chi_{0}, \chi_{1}\right)$ defines a morphism $\left(d_{0}, \eta, d_{1}\right) \longrightarrow\left(d_{0}^{\prime}, \eta^{\prime}, d_{1}^{\prime}\right)$. That is, we need to prove that $F_{1}\left(\chi_{1}\right) \circ \eta=\eta^{\prime} \circ F_{0}\left(\chi_{0}\right)$ in E. So far we have a diagram

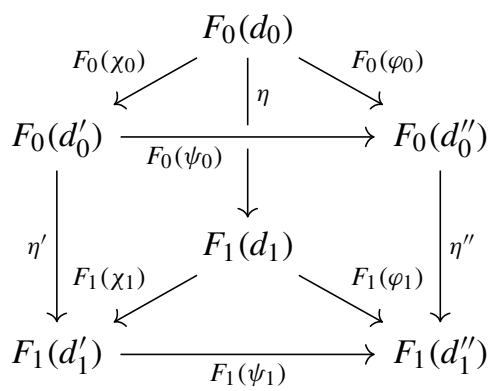

in $E$, where each face of this diagram commutes, apart from the back left square. The commutativity of that square is what we need to prove. For this, observe that both $\eta^{\prime} \circ F_{0}\left(\chi_{0}\right)$ and $\eta \circ F_{1}\left(\chi_{1}\right)$ provide lifts of $\chi$ to $\mathrm{E}$ with respect to the morphisms $\eta^{\prime \prime} \circ F_{0}\left(\varphi_{0}\right): F_{0}\left(d_{0}\right) \longrightarrow F_{1}\left(d_{1}^{\prime \prime}\right)$ and $F_{1}\left(\psi_{1}\right): F_{1}\left(d_{1}^{\prime}\right) \longrightarrow F_{1}\left(d_{1}^{\prime \prime}\right)$. The desired identity now follows from the uniqueness of such lifts along the functor $\pi_{\mathrm{E}}$.

Next we need to show that for any morphism $f: c \longrightarrow c^{\prime}$ in $\mathcal{C}$ and any object $\left(d_{0}^{\prime}, \eta^{\prime}, d_{1}^{\prime}\right)$ in $\mathrm{D}_{0} \times \mathrm{E}_{\mathrm{E}}^{\mathrm{h}} \mathrm{D}_{1}$ over $c^{\prime}$, there exists a lift $\widehat{f}=\left(f_{0}, f_{1}\right)$ of $f$ to $\mathrm{D}_{0} \times \mathrm{E}_{\mathrm{E}}^{\mathrm{h}} \mathrm{D}_{1}$ with codomain $\left(d_{0}^{\prime}, \eta^{\prime}, d_{1}^{\prime}\right)$. Such a lift is obtained by lifting $f$ to morphisms $f_{i}: d_{i} \longrightarrow d_{i}^{\prime}$ in $\mathrm{D}_{i}$ using the fact that $\pi_{i}$ is a Grothendieck fibration in groupoids, for $i=0,1$. An isomorphism $\eta: F_{0}\left(d_{0}\right) \longrightarrow F_{1}\left(d_{1}\right)$ compatible with $f_{0}, f_{1}$ is obtained by filling the horn given by the morphisms $\eta^{\prime} \circ F_{0}\left(f_{0}\right)$ and $F_{1}\left(f_{1}\right)$ over the identity morphism $1_{c}$ in $\mathcal{C}$. The filler is an isomorphism since the fibre $\mathrm{E}_{\mid c}$ is a groupoid.

To prove (2), we note that by [Vis05, Proposition 3.36] the induced morphism $G_{0} \times{ }_{G_{\mathrm{E}}}^{\mathrm{h}}$ $G_{1}$ is an equivalence in $\mathcal{H}$ if and only if it restricts to an equivalence of groupoids between all fibres of $\pi_{\mathrm{h}}$ and $\pi_{\mathrm{h}}^{\prime}$. A direct inspection on any $c \in \mathcal{C}$ reveals that

$$
\pi_{\mathrm{h}}^{-1}(c)=\pi_{0}^{-1}(c) \times_{\pi_{\mathrm{E}}^{-1}(c)}^{\mathrm{h}} \pi_{1}^{-1}(c)
$$

as groupoids, and it is well-known that equivalences of spans of groupoids induce equivalences on homotopy pullbacks of groupoids. 
To prove (3), we first show that $\mathrm{pr}_{0}^{\mathrm{h}}$ is a Grothendieck fibration in groupoids. Consider a span

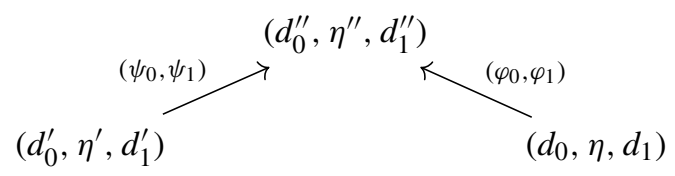

in $\mathrm{D}_{0} \times \times_{\mathrm{E}}^{\mathrm{h}} \mathrm{D}_{1}$, and a morphism $\chi_{0}: d_{0} \longrightarrow d_{0}^{\prime}$ in $\mathrm{D}_{0}$ such that $\psi_{0} \circ \chi_{0}=\varphi_{0}$. We obtain a commutative triangle in $\mathrm{E}$ formed by the morphisms $F_{1}\left(\psi_{1}\right), F_{1}\left(\varphi_{1}\right)$, and $\eta^{\prime} \circ F_{0}\left(\chi_{0}\right) \circ \eta^{-1}$. Since $F_{1}$ is a Grothendieck fibration in groupoids, this gives rise to a unique lift $\chi_{1}: d_{1} \longrightarrow d_{1}^{\prime}$ of the latter morphism to $D_{1}$. The pair $\left(\chi_{0}, \chi_{1}\right)$ is automatically a morphism in $D_{0} \times_{E}^{h} D_{1}$ which projects to $\chi_{0}$. If $\left(\chi_{0}^{\prime}, \chi_{1}^{\prime}\right)$ were any other such filling over $\chi_{0}$ of the horn given by $\left(\psi_{0}, \psi_{1}\right)$ and $\left(\varphi_{0}, \varphi_{1}\right)$, it would immediately follow that $\chi_{0}^{\prime}=\chi_{0}$, and the uniqueness of fillings of $\psi_{1}, \varphi_{1}$ over $\eta^{\prime} \circ F_{0}\left(\chi_{0}\right) \circ \eta^{-1}$ would imply that $\chi_{1}^{\prime}=\chi_{1}$.

Let $\varphi_{0}: d_{0} \longrightarrow d_{0}^{\prime}$ be a morphism in $\mathrm{D}_{0}$, and let $\left(d_{0}^{\prime}, \eta^{\prime}, d_{1}^{\prime}\right)$ be an object in $\mathrm{D}_{0} \times{ }_{\mathrm{E}}^{\mathrm{h}} \mathrm{D}_{1}$ which projects to $d_{0}^{\prime}$. Let $\varphi_{1}$ be a lift along $\pi_{1}$ of $f:=\pi_{0}\left(\varphi_{0}\right)$ with codomain $d_{1}^{\prime}$. The pair of morphisms $\left(\eta^{\prime} \circ F_{0}\left(\psi_{0}\right), F_{1}\left(\psi_{1}\right)\right)$ then gives rise to a cospan in $\mathrm{E}$. Both morphisms project to $f$ in $\mathcal{C}$ and hence, since $\pi_{\mathrm{E}}$ is a Grothendieck fibration in groupoids, there exists a unique isomorphism $\eta: F_{0}\left(d_{0}\right) \longrightarrow F_{1}\left(d_{1}\right)$ such that $\left(d_{0}, \eta, d_{1}\right) \in \mathrm{D}_{0} \times \mathrm{E}_{\mathrm{E}}^{\mathrm{h}} \mathrm{D}_{1}$ and such that $\left(\psi_{0}, \psi_{1}\right)$ is a lift of $\psi_{0}$ to $\mathrm{D}_{0} \times_{\mathrm{E}}^{\mathrm{h}} \mathrm{D}_{1}$ with codomain $\left(d_{0}^{\prime}, \eta^{\prime}, d_{1}^{\prime}\right)$. The claim for $\mathrm{pr}_{0}$ is proven in an entirely analogous way by restricting $\eta, \eta^{\prime}$ and $\eta^{\prime \prime}$ to be identity morphisms.

Finally, consider the inclusion functor $D_{0} \times_{E} D_{1} \hookrightarrow D_{0} \times D_{E}^{h}$. Since $\operatorname{pr}_{0}^{h}$ is a Grothendieck fibration in groupoids, so is its restriction to each fibre over $\mathcal{C}$. It is wellknown that the inclusion of a pullback of groupoids into the homotopy pullback is an equivalence in case one of the functors in the diagram is a Grothendieck fibration. Thus our inclusion functor is an equivalence on each fibre over $\mathcal{C}$, whence the result follows by [Vis05, Proposition 3.36].

A.2. Relation to principal $\infty$-bundles. Our notion of smooth principal 2-bundle does not have any notion of 'local triviality' built into it. This differs from the version of a principal 2-bundle defined in [SP11], but is very much in the spirit of the definition of a principal $\infty$-bundle from [NSS15]. The fact that we require essential surjectivity is our version of saying that the (homotopy) fibres of the bundle should be non-empty. In contrast to [NSS15] we have to require fibration properties because we do not work purely in an $\infty$-categorical framework. We shall now show that an $\mathrm{H}$-principal 2-bundle in $\mathcal{H}$ in the sense of Definition 5.17 gives rise to a principal 2-bundle in the sense of [NSS15, Definition 3.4], adapted from a general $\infty$-topos (described e.g. by presheaves of $\infty$ groupoids) to our situation involving presheaves of groupoids. Let $p: \mathrm{P} \longrightarrow \mathrm{X}$ be a morphism in $\mathcal{H}$, and let $\mathrm{P}^{[\bullet]}$ be the Čech nerve of $p$. We write hocolim ${ }^{\mathcal{C}}$ (resp. holim ${ }^{\mathrm{C}}$ ) for a homotopy colimit (resp. limit) taken in a simplicial model category $\mathrm{C}$.

Proposition A.3. Every morphism $p: \mathrm{P} \longrightarrow \mathrm{X}$ in $\mathcal{H}$ whose underlying functor is an essentially surjective Grothendieck fibration in groupoids gives rise to an effective epimorphism: the morphism

$$
\underset{\Delta^{\mathrm{op}}}{\operatorname{hocolim}}{ }^{\mathcal{H}} \mathrm{P}^{[\bullet]} \longrightarrow \mathrm{X}
$$


from its $\check{C}$ ech nerve to $\mathrm{X}$ is an equivalence.

Because of Lemma A.2 and the assumption that $p$ is a Grothendieck fibration in groupoids, it does not matter here if one uses the coherent Čech nerve, formed using $P \times_{x}^{h} \cdots x_{x}^{h} P$, or the strict Čech nerve, formed using $P \times_{x} \cdots \times x P$.

Proof. We work with Hollander's model structure on $\mathcal{H}$ [Hol08]. In this picture, $\mathcal{H}$ is a model category enriched, tensored and cotensored in the model category Grpd (seen as a strict category). In both $\mathcal{H}$ and $\mathcal{G}$ rpd all objects are fibrant, and the functor $\underline{\mathcal{H}}: \mathcal{H}^{\mathrm{op}} \times \mathcal{H} \longrightarrow \mathcal{G r p d}$ is homotopical by [Vis05, Proposition 3.35], i.e. it preserves weak equivalences in each argument. The enrichment of $\mathcal{H}$ in $\mathcal{G r p d}$ even enhances to an enrichment over $\operatorname{Set}_{\Delta}$, the category of simplicial sets with the Kan-Quillen model structure. Thus homotopy (co)limits in $\mathcal{H}$ can be computed using (co)bar constructions [Rie14]. Let $Q$ denote a cofibrant replacement functor in $\mathcal{H}$, and let $\mathbf{Z} \in \mathcal{H}$ be an arbitrary object. Then

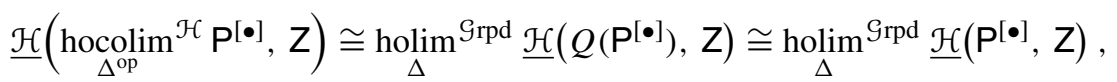

where the first equivalence stems from the fact that $Z$ is fibrant and $\mathcal{H}$ is a $\mathcal{G r p d}$-enriched model category, and the second equivalence stems from the fact that $\underline{\mathcal{H}}$ is homotopical. It thus suffices to prove that the functor

$$
p^{*}: \underline{\mathcal{H}}(\mathbf{X}, \mathbf{Z}) \longrightarrow \underset{\Delta}{\operatorname{holim}} \operatorname{Grpd} \underline{\mathcal{H}}\left(\mathrm{P}^{[\bullet]}, \mathbf{Z}\right)=: \mathfrak{D e s}_{p}(\mathrm{Z})
$$

is an equivalence of groupoids.

An object in $\mathfrak{D e s}_{p}(Z)$ is a pair $(G, \eta)$ of a functor $G: \mathrm{P} \longrightarrow \mathrm{Z}$ of categories fibred in groupoids over Cart, together with a natural isomorphism $\eta_{\mid\left(p_{0}, p_{1}\right)}: G\left(p_{0}\right) \longrightarrow G\left(p_{1}\right)$ from $d_{1}^{*} G$ to $d_{0}^{*} G$ of functors over Cart, where $d_{i}$ are the face maps in the simplicial object $\mathrm{P}^{[\bullet]}$. This natural isomorphism is subject to the conditions $d_{2}^{*} \eta \circ d_{0}^{*} \eta=d_{1}^{*} \eta$ over $\mathrm{P}^{[3]}$ and $\Delta^{*} \eta=1_{G}$ over $\mathrm{P}$, where $\Delta: \mathrm{P} \longrightarrow \mathrm{P}^{[2]}$ is the diagonal map. A morphism $(G, \eta) \longrightarrow\left(G^{\prime}, \eta^{\prime}\right)$ in $\mathfrak{D e s}_{p}(\mathbf{Z})$ is a natural isomorphism $\gamma: G \longrightarrow G^{\prime}$ in $\mathcal{H}$ such that $\eta^{\prime} \circ d_{1}^{*} \gamma=d_{0}^{*} \gamma \circ \eta$.

We first show that $p^{*}$ is essentially surjective: let $(G, \eta) \in \mathfrak{D e s}_{p}(Z)$ be any object. We define a functor $F: X \longrightarrow Z$ as follows: first, recalling that $p$ is surjective on objects by Lemma A.1, we choose a section $s: \mathrm{ob}(\mathrm{X}) \longrightarrow \mathrm{ob}(\mathrm{P})$ of the map of objects defined by $p$. Then we set $F(x):=G(s(x)) \in \mathrm{Z}$ for $x \in \mathrm{X}$. Now consider a morphism $\psi: x \longrightarrow y$ in X. Since $p$ is a Grothendieck fibration, $\psi$ has a lift $\widehat{\psi}: \widehat{x} \longrightarrow s(y)$ to a morphism in $\mathrm{P}$ with codomain $s(y)$, where $p(\widehat{x})=x$. Define $F(\psi): F(x) \longrightarrow F(y)$ via the diagram

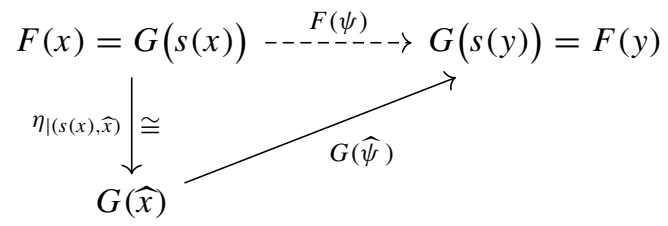

The naturality of $\eta$, together with the two conditions it satisfies and the fact that $p$ is a Grothendieck fibration in groupoids, imply that $F$ is a well-defined functor. Furthermore, $\eta$ establishes an isomorphism $p^{*} F=\left(F, 1_{F}\right) \longrightarrow(G, \eta)$ in $\mathfrak{D e s}_{p}(\mathrm{Z})$. Thus $p^{*}$ is essentially surjective.

That $p^{*}$ is fully faithful follows from its explicit construction and the fact that $p$ is essentially surjective. 


\section{References}

[Alf20] Alfonsi, L.: Global double field theory is higher Kaluza-Klein theory. Fortsch. Phys. 68, 2000010,42 (2020). arXiv: 1912.07089

[AS84] Atiyah, M.F., Singer, I.M.: Dirac operators coupled to vector potentials. Proc. Natl. Acad. Sci. USA 81, 2597-2600 (1984)

[BCSS07] Baez, J.C., Crans, A., Schreiber, U., Stevenson, D.: From loop groups to 2-groups. Homol. Homot. Appl. 9(2), 101-135 (2007). arXiv:math/0504123

[BL04] Baez, J.C., Lauda, A.D.: Higher-dimensional algebra V. 2-groups. Theory Appl. Categ. 12, 423491 (2004). arXiv:math/0307200

[BH11] Baez, J.C., Hoffnung, A.E.: Convenient categories of smooth spaces. Trans. Am. Math. Soc. 363(11), 5789-5825 (2011). arXiv:0807.1704

[BL14] Bakas, I., Lüst, D.: 3-cocycles, nonassociative star products and the magnetic paradigm of $R$-flux string vacua. J. High Energy Phys. 01, 171,49 (2014). arXiv:1309.3172

[BP11] Blumenhagen, R., Plauschinn, E.: Nonassociative gravity in string theory? J. Phys. A 44, 015401,19 (2011). arXiv:1010.1263

[BBBS15] Bojowald, M., Brahma, S., Büyükçam, U., Strobl, T.: States in nonassociative quantum mechanics: Uncertainty relations and semiclassical evolution. J. High Energy Phys. 03, 093,22 (2015). arXiv: 1411.3710

[BMS19] Bunk, S., Müller, L., Szabo, R.J.: Geometry and 2-Hilbert space for nonassociative magnetic translations. Lett. Math. Phys. 109(8), 1827-1866 (2019). arXiv:1804.08953

[BSS18] Bunk, S., Saemann, C., Szabo, R.J.: The 2-Hilbert space of a prequantum bundle gerbe. Rev. Math. Phys. 30(1), 1850001,101 (2018). arXiv:1608.08455

[BS17] Bunk, S., Szabo, R.J.: Fluxes, bundle gerbes and 2-Hilbert spaces. Lett. Math. Phys. 107(10), 1877-1918 (2017). arXiv:1612.01878

[Bun17] Bunk, S.: Categorical structures on bundle Gerbes and higher geometric prequantisation. PhD Thesis, Heriot-Watt University, Edinburgh, 139 pp (2017). arXiv:1709.06174

[Bun20a] Bunk, S.: Sheaves of higher categories and presentations of smooth field theories. Preprint, $50 \mathrm{pp}$. (2020). arXiv:2003.00592

[Bun20b] Bunk, S.: Principal $\infty$-bundles and smooth string group models. Preprint, 44 pp. (2020). arXiv:2008.12263

[BW18] Bunk, S., Waldorf, K.: Transgression of D-branes. Preprint, 69 pp. (2018). arXiv:1808.04894

[BW19] Bunk, S., Waldorf, K.: Smooth functorial field theories from $B$-fields and D-branes. Preprint, 64 pp. (2019). arXiv:1911.09990

[Bre93] Bredon, G. E.: Topology and Geometry. Springer Graduate Texts in Mathematics (1993)

[CMM97] Carey, A.L., Mickelsson, J., Murray, M.K.: Index theory, Gerbes, and Hamiltonian quantization. Commun. Math. Phys. 183, 707-722 (1997). arXiv:hep-th/9511151

[CMM00] Carey, A.L., Mickelsson, J., Murray, M.K.: Bundle Gerbes applied to quantum field theory. Rev. Math. Phys. 12(1), 65-90 (2000). arXiv:hep-th/9711133

[CM95] Carey, A.L., Murray, M.K.: Mathematical remarks on the cohomology of gauge groups and anomalies. In: Confronting the Infinite, pp. 136-148. World Scientific Publishing, River Edge, NJ (1995). arXiv:hep-th/9408141

[CM96] Carey, A.L., Murray, M.K.: Faddeev's anomaly and bundle gerbes. Lett. Math. Phys. 37(1), 29-36 (1996)

[Col11] Collier, B.L.: Infinitesimal symmetries of Dixmier-Douady gerbes. Preprint (2011). arXiv: 1108.1525

[DGTS20] Davighi, J., Gripaios, B., Tooby-Smith, J.: Quantum mechanics in magnetic backgrounds with manifest symmetry and locality. J. Phys. A 53, 145302,35 (2020). arXiv:1905.11999

[DHH11] Douglas, C.L., Henriques, A.G., Hill, M.A.: Homological obstructions to string orientations. Int. Math. Res. Not. 18, 4074-4088 (2011). arXiv:0810.2131

[Fad84] Faddeev, L.D.: Operator anomaly for the Gauss law. Phys. Lett. B 145, 81-84 (1984)

[FS85] Faddeev, L.D., Shatashvili, S.L.: Algebraic and Hamiltonian methods in the theory of nonabelian anomalies. Theor. Math. Phys. 60, 770-778 (1985)

[Fio13] Fiore, G.: On quantum mechanics with a magnetic field on $\mathbb{R}^{n}$ and on a torus $\mathbb{T}^{n}$. Int. J. Theor. Phys. 52, 877-896 (2013). arXiv:1103.0034

[FRS16] Fiorenza, D., Rogers, C.L., Schreiber, U.: Higher U(1)-gerbe connections in geometric prequantisation. Rev. Math. Phys. 28(6), 1650012,72 (2016). arXiv:1304.0236

[GSW11] Gawędzki, K., Suszek, R., Waldorf, K.: Global gauge anomalies in two-dimensional bosonic sigma models. Commun. Math. Phys. 302(2), 513-580 (2011). arXiv:1003.4154

[Gru00] Gruber, M.J.: Bloch theory and quantisation of magnetic systems. J. Geom. Phys. 34, 137-154 (2000). arXiv:math-ph/9903048 
[GZ86] Günaydin, M., Zumino, B.: Magnetic charge and nonassociative algebras. In: Old and New Problems in Fundamental Physics: Meeting in Honour of G. C. Wick, pp. 43-53. Quaderni, Pisa: Scuola Normale Superiore (1986)

[Hol08] Hollander, S.: A homotopy theory for stacks. Israel J. Math. 163, 93-124 (2008). arXiv:math/0110247

[IZ13] Iglesias-Zemmour, P.: Diffeology. Mathematical Surveys and Monographs, 185. American Mathematical Society (2013)

[Jac85] Jackiw, R.: 3-cocycle in mathematics and physics. Phys. Rev. Lett. 54, 159-162 (1985)

[Jo85] Jo, S.-G.: Commutators in an anomalous nonabelian chiral gauge theory. Phys. Lett. B 163, 353359 (1985)

[KS18] Kupriyanov, V.G., Szabo, R.J.: Symplectic realisation of electric charge in fields of monopole distributions. Phys. Rev. D 98(4), 045005,25 (2018). arXiv:1803.00405

[Lee13] Lee, J.M.: Introduction to Smooth Manifolds, 2nd edn. Springer Graduate Texts in Mathematics (2013)

[Lur09] Lurie, J.: Higher topos theory. Ann. Math. Stud. 170, 1-925 (2009). arXiv:math/0806040

[Lüs10] Lüst, D.: T-duality and closed string noncommutative (doubled) geometry. J. High Energy Phys. 12, 08428 (2010). arXiv:1010.1361

[Mic85] Mickelsson, J.: Chiral anomalies in even and odd dimensions. Commun. Math. Phys. 97, 361-370 (1985)

[Mic19] Mickelsson, J.: Nonassociative magnetic translations: a QFT construction. Preprint (2019). arXiv:1905.01944

[MW16] Mickelsson, J., Wagner, S.: Third group cohomology and gerbes over Lie groups. J. Geom. Phys. 108, 49-70 (2016). arXiv:1602.02565

[Moe02] Moerdijk, I.: Introduction to the language of stacks and gerbes. Preprint (2002). arXiv:math/0212266

[Mur96] Murray, M.K.: Bundle gerbes. J. Lond. Math. Soc. 54, 403-416 (1996). arXiv:dg-ga/9407015

[M+17] Murray, M.K., Roberts, D.M., Stevenson, D., Vozzo, R.F.: Equivariant bundle gerbes. Adv. Theor. Math. Phys. 21(4), 921-975 (2017). arXiv:1506.07931

[MSS12] Mylonas, D., Schupp, P., Szabo, R.J.: Membrane sigma-models and quantisation of non-geometric flux backgrounds. J. High Energy Phys. 09, 012,55 (2012). arXiv:1207.0926

[MSS14] Mylonas, D., Schupp, P., Szabo, R.J.: Non-geometric fluxes, quasi-Hopf twist deformations and nonassociative quantum mechanics. J. Math. Phys. 55, 122301,30 (2014). arXiv:1312.1621

[NSW13] Nikolaus, T., Sachse, C., Wockel, C.: A smooth model for the string group. Int. Math. Res. Not. 16, 3678-3721 (2013). arXiv:1104.4288

[NSS15] Nikolaus, T., Schreiber, U., Stevenson, D.: Principal $\infty$-bundles: General theory. J. Homot. Relat. Struct. 10(4), 749-801 (2015). arXiv:1207.0248

[NS11] Nikolaus, T., Schweigert, C.: Equivariance in higher geometry. Adv. Math. 226(4), 3367-3408 (2011). arXiv:1004.4558

[Rie14] Riehl, E.: Categorical Homotopy Theory. New Mathematical Monographs, 24. Cambridge University Press (2014)

[SS20] Saemann, C., Schmidt, L.: The nonabelian self-dual string. Lett. Math. Phys. 110, 1001-1042 (2020). arXiv: 1705.02353

[SP11] Schommer-Pries, C.J.: Central extensions of smooth 2-groups and a finite-dimensional string 2-group. Geom. Topol. 15(2), 609-676 (2011). arXiv:0911.2483

[Sch13] Schreiber, U.: Differential cohomology in a cohesive $\infty$-topos. Preprint. arXiv:1310.7930

[SW09] Schreiber, U., Waldorf, K.: Parallel transport and functors. J. Homot. Relat. Struct. 4(1), 187-244 (2009). arXiv:0705.0452

[SW11] Schreiber, U., Waldorf, K.: Smooth functors vs. differential forms. Homol. Homot. Appl. 13(1), 143-203 (2011). arXiv:0802.0663

[SW17] Schreiber, U., Waldorf, K.: Local theory for 2-functors on path 2-groupoids. J. Homot. Relat. Struct. 12(3), 617-658 (2017). arXiv:1303.4663

[Sol18] Soloviev, M.A.: Dirac's magnetic monopole and the Kontsevich star product. J. Phys. A 51(9), 095205,21 (2018). arXiv:1708.05030

[Sto96] Stolz, S.: A conjecture concerning positive Ricci curvature and the Witten genus. Math. Ann. 304(4), 785-800 (1996)

[ST04] Stolz, S., Teichner, P.: What is an elliptic object? Lond. Math. Soc. Lect. Note Ser. 308, 247-343 (2004)

[Sza13] Szabo, R.J.: Quantisation of higher abelian gauge theory in generalised differential cohomology. Proc. Sci. 175, 009,65 (2013). arXiv: 1209.2530

[Sza19a] Szabo, R.J.: Quantisation of magnetic Poisson structures. Fortsch. Phys. 67(8-9), 1910022,13 (2019). arXiv:1903.02845 
[Sza19b] Szabo, R.J.: An introduction to nonassociative physics. Proc. Sci. 347, 100,41 (2019). arXiv: 1903.05673

[Vis05] Vistoli, A.: Grothendieck topologies, fibred categories and descent theory. Math. Surv. Monogr. 123, 1-104 (2005). arXiv:math/0412512

[Wa107a] Waldorf, K.: Algebraic structures for bundle gerbes and the Wess-Zumino term in conformal field theory. PhD Thesis, Universität Hamburg, 179 pp. (2007)

[Wa107b] Waldorf, K.: More morphisms between bundle gerbes. Theory Appl. Categ. 18(9), 240-273 (2007). arXiv:math/0702652

[Wal12a] Waldorf, K.: A construction of string 2-group models using a transgression-regression technique. Contemp. Math. 584, 99-115 (2012). arXiv:1201.5052

[Wal12b] Waldorf, K.: Transgression to loop spaces and its inverse I: diffeological bundles and fusion maps. Cah. Topol. Géom. Différ. Catég. LIII, 162-210 (2012). arXiv:0911.3212

[Wal16] Waldorf, K.: Transgression to loop spaces and its inverse II: gerbes and fusion bundles with connection. Asian J. Math. 20(1), 59-115 (2016). arXiv:1004.0031

[Wal18] Waldorf, K.: Parallel transport in principal 2-bundles. High. Struct. 2(1), 57-115 (2018). arXiv: 1704.08542

Communicated by H.-T. Yau 